\title{
SERRE WEIGHTS AND WILD RAMIFICATION IN TWO-DIMENSIONAL GALOIS REPRESENTATIONS
}

\author{
LASSINA DEMBÉLÉ ${ }^{1}$, FRED DIAMOND $^{2}$ and DAVID P. ROBERTS ${ }^{3}$ \\ ${ }^{1}$ Mathematics Institute, University of Warwick, Coventry CV4 7AL, UK; \\ email: lassina.dembele@gmail.com \\ ${ }^{2}$ Department of Mathematics, King's College London, London WC2R 2LS, UK; \\ email: fred.diamond@kcl.ac.uk \\ ${ }^{3}$ Division of Science and Mathematics, University of Minnesota Morris, Morris, MN 56267, USA; \\ email: roberts@morris.umn.edu
}

Received 29 June 2016; accepted 20 October 2016

\begin{abstract}
A generalization of Serre's Conjecture asserts that if $F$ is a totally real field, then certain characteristic $p$ representations of Galois groups over $F$ arise from Hilbert modular forms. Moreover, it predicts the set of weights of such forms in terms of the local behaviour of the Galois representation at primes over $p$. This characterization of the weights, which is formulated using $p$-adic Hodge theory, is known under mild technical hypotheses if $p>2$. In this paper we give, under the assumption that $p$ is unramified in $F$, a conjectural alternative description for the set of weights. Our approach is to use the Artin-Hasse exponential and local class field theory to construct bases for local Galois cohomology spaces in terms of which we identify subspaces that should correspond to ones defined using $p$-adic Hodge theory. The resulting conjecture amounts to an explicit description of wild ramification in reductions of certain crystalline Galois representations. It enables the direct computation of the set of Serre weights of a Galois representation, which we illustrate with numerical examples. A proof of this conjecture has been announced by Calegari, Emerton, Gee and Mavrides.
\end{abstract}

2010 Mathematics Subject Classification: 11F80 (primary); 11S15, 11S25 (secondary)

\section{Introduction}

A conjecture of Serre [24], now a theorem of Khare and Wintenberger $[17,18]$, asserts that if $p$ is prime and

$$
\rho: G_{\mathbf{Q}} \rightarrow \mathrm{GL}_{2}\left(\overline{\mathbf{F}}_{p}\right)
$$

(c) The Author(s) 2016. This is an Open Access article, distributed under the terms of the Creative Commons Attribution licence (http://creativecommons.org/licenses/by/4.0/), which permits unrestricted re-use, distribution, and reproduction in any medium, provided the original work is properly cited. 
is a continuous, odd, irreducible representation, then $\rho$ arises from a Hecke eigenform in the space $S_{k}\left(\Gamma_{1}(N)\right)$ of cusp forms of some weight $k$ and level $N$. Serre in fact formulated a refined version of the conjecture specifying the minimal such $k$ and $N$ subject to the constraints $k \geqslant 2$ and $p \nmid N$; a key point is that the weight depends only on the restriction of $\rho$ to a decomposition group at $p$, and the level on ramification away from $p$. The equivalence between the weaker version of the conjecture and its refinement was already known through the work of many authors for $p>2$, and finally settled for $p=2$ as well by Khare and Wintenberger.

Buzzard, Jarvis and one of the authors [4] considered a generalization of Serre's conjecture to the setting of Hilbert modular forms for a totally real number field $F$ and formulated an analogous refinement for representations $\rho: G_{F} \rightarrow \mathrm{GL}_{2}\left(\overline{\mathbf{F}}_{p}\right)$ assuming $p$ is unramified in $F$; versions without this assumption are given in [22] and [12]. The equivalence between the conjecture and its refinement was proved, assuming $p>2$ and a Taylor-Wiles hypothesis on $\rho$, in a series of papers by Gee and several sets of co-authors culminating in [15] and [14], with an alternative to the latter provided by Newton [20]. Generalizations to higher-dimensional Galois representations have also been studied by Ash, Herzig and others beginning with [2]; see [13] for recent development.

One of the main difficulties in even formulating refined versions of generalizations of Serre's conjecture is in prescribing the weights; the approach taken in [4] and subsequent papers, at least if $\rho$ is wildly ramified at primes over $p$, is to do this in terms of Hodge-Tate weights of crystalline lifts of $\rho$. The main purpose of this paper is to make the recipe for the set of weights more explicit. In view of the connection between Serre weights and crystalline lifts, this amounts to a conjecture in explicit $p$-adic Hodge theory about wild ramification in reductions of crystalline Galois representations.

We now explain this in more detail. Let $F$ be a totally real number field, $\mathcal{O}$ its ring of integers, $\mathfrak{n}$ a nonzero ideal of $\mathcal{O}, S_{F}$ the set of embeddings $F \rightarrow \mathbf{R}$ and suppose $\vec{k} \in \mathbf{Z}^{S_{F}}$ with all $k_{\tau} \geqslant 2$ and of the same parity. A construction completed by Taylor in [25] then associates a $p$-adic Galois representation to each Hecke eigenform in the space of Hilbert modular cusp forms of weight $\vec{k}$ and level $\mathfrak{n}$. One then expects that every continuous, irreducible, totally odd

$$
\rho: G_{F} \rightarrow \mathrm{GL}_{2}\left(\overline{\mathbf{F}}_{p}\right)
$$

is modular in the sense that it arises as the reduction of such a Galois representation. One further expects that the prime-to- $p$ part of the minimal level from which $\rho$ arises is its Artin conductor, but the prediction of the possible weights is more subtle. If $p$ is unramified in $F$, then a recipe is given in [4] in terms of the restrictions of $\rho$ to decomposition groups at primes $\mathfrak{p}$ over $p$. An 
interesting feature of this recipe not so apparent over $\mathbf{Q}$ is the dependence of the conjectured weights on the associated local extension class when the restriction at $\mathfrak{p}$ is reducible. If

$$
\left.\rho\right|_{G_{F_{\mathfrak{p}}}} \sim\left(\begin{array}{cc}
\chi_{1} & * \\
0 & \chi_{2}
\end{array}\right)
$$

for some characters $\chi_{1}, \chi_{2}: G_{F_{\mathfrak{p}}} \rightarrow \overline{\mathbf{F}}_{p}^{\times}$, then the resulting short exact sequence

$$
0 \rightarrow \overline{\mathbf{F}}_{p}\left(\chi_{1}\right) \rightarrow V_{\rho} \rightarrow \overline{\mathbf{F}}_{p}\left(\chi_{2}\right) \rightarrow 0
$$

defines a class in

$$
c_{\rho} \in \operatorname{Ext}_{\overline{\mathbf{F}}_{p}\left[G_{K}\right]}^{1}\left(\overline{\mathbf{F}}_{p}\left(\chi_{2}\right), \overline{\mathbf{F}}_{p}\left(\chi_{1}\right)\right) \cong \operatorname{Ext}_{\overline{\mathbf{F}}_{p}\left[G_{K}\right]}^{1}\left(\overline{\mathbf{F}}_{p}, \overline{\mathbf{F}}_{p}(\chi)\right) \cong H^{1}\left(G_{K}, \overline{\mathbf{F}}_{p}(\chi)\right)
$$

where $K=F_{\mathfrak{p}}$ and $\chi=\chi_{1} \chi_{2}^{-1}$. The class $c_{\rho}$ is well defined up to a scalar in $\overline{\mathbf{F}}_{p}^{\times}$, in the sense that another choice of basis with respect to which $\left.\rho\right|_{G_{K}}$ has the form (1) yields a nonzero scalar multiple of $c_{\rho}$. Alternatively, we may view $c_{\rho}$ as the class in $H^{1}\left(G_{K}, \overline{\mathbf{F}}_{p}(\chi)\right)$ defined by the cocycle $z$ obtained by writing

$$
\left.\chi_{2}^{-1} \otimes \rho\right|_{G_{K}} \sim\left(\begin{array}{ll}
\chi & z \\
0 & 1
\end{array}\right) .
$$

The space $H^{1}\left(G_{K}, \overline{\mathbf{F}}_{p}(\chi)\right)$ has dimension at least $\left[K: \mathbf{Q}_{p}\right]$, with equality unless $\chi$ is trivial or cyclotomic. Whether $\rho$ is modular of a particular weight depends on whether this extension class lies in a certain distinguished subspace of $H^{1}\left(G_{K}, \overline{\mathbf{F}}_{p}(\chi)\right)$ whose definition relies on constructions from $p$-adic Hodge theory. If $K \neq \mathbf{Q}_{p}$ and $\rho$ is wildly ramified at $\mathfrak{p}$, then the associated extension class is a nontrivial element of a space of dimension at least two, making it difficult to determine the set of weights without a more explicit description of the distinguished subspaces.

We address the problem in this paper by using local class field theory and the Artin-Hasse exponential to give an explicit basis for the space of extensions (Corollary 5.2), in terms of which we provide a conjectural alternate description of the distinguished subspaces (Conjecture 7.2). We point out that a related problem is considered by Abrashkin in [1]; in particular, the results of [1] imply cases of our conjecture where the distinguished subspaces can be described using the ramification filtration on $G_{K}$.

An earlier version of this paper was posted on the arXiv in March 2016. At the time, we reported that a proof of Conjecture 7.2 under certain genericity hypotheses would be forthcoming in the Ph.D thesis of Mavrides [19]. In fact, Conjecture 7.2 has now been proved completely by Calegari, Emerton, Gee, and 
Mavrides in a preprint posted to the arXiv in August 2016 [5]. We remark that our restriction to the case where $K$ is unramified over $\mathbf{Q}_{p}$ is made essentially for simplicity. The methods of this paper, and indeed of [5], are expected to apply to the general case where $K / \mathbf{Q}_{p}$ is allowed to be ramified, but the resulting explicit description of the distinguished subspaces is likely to be much more complicated.

The now-proved Conjecture 7.2 immediately yields an alternate description of the set of Serre weights for $\rho$. Combining this with the predicted modularity of $\rho$ gives Conjecture 7.3, for which we have gathered extensive computational evidence. Indeed the appeal of our description is that one can compute the set of Serre weights directly from $\rho$. In this paper, we illustrate this computation systematically in several examples with $K / \mathbf{Q}_{p}$ quadratic and $p=3$. A sequel paper [9] will support Conjecture 7.3 via a much broader range of examples and elaborate on computational methods. In particular, the examples provided in [9] illustrate subtle features of the recipe for the weights arising only when $\chi$ is highly nongeneric, with particular attention to the case $p=2$. Such examples were instrumental in leading us to Conjecture 7.2 in its full generality.

This paper is structured as follows: In Section 2 we recall the general statement of the weight part of Serre's conjecture for $F$ unramified at $p$. In Sections 3, 4 and 5, we study the space of extensions $H^{1}\left(G_{K}, \overline{\mathbf{F}}_{p}(\chi)\right)$ in detail, arriving at an explicit basis in terms of the Artin-Hasse exponential. In Sections 6 and 7 , we use this basis to give our conjectural description of the distinguished subspaces appearing in the definition of the set of Serre weights. We illustrate this description in more detail in the quadratic case in Section 8, and with numerical examples for $p=3$ in Sections 9 and 10. We remark that aside from these examples and the discussion of Serre's conjecture at the end of Sections 2 and 7, the setting for the paper is entirely local.

\section{Serre weights}

2.1. Notation and general background. Let $K$ be an unramified extension of $\mathbf{Q}_{p}$ with ring of integers $\mathcal{O}_{K}$ and residue field $k$, and let $f=\left[K: \mathbf{Q}_{p}\right]=\left[k: \mathbf{F}_{p}\right]$. We fix algebraic closures $\overline{\mathbf{Q}}_{p}$ and $\bar{K}$ of $\mathbf{Q}_{p}$ and $K$, and let $T$ denote the set of embeddings $K \rightarrow \overline{\mathbf{Q}}_{p}$. We let $\overline{\mathbf{F}}_{p}$ denote the algebraic closure $\mathbf{F}_{p}$ obtained as the residue field of the ring of integers of $\overline{\mathbf{Q}}_{p}$, and we identify $T$ with the set of embeddings $k \rightarrow \overline{\mathbf{F}}_{p}$ via the canonical bijection.

For a field $F$, we write $G_{F}$ for the absolute Galois group of $F$. We let $I_{K}$ denote the inertia subgroup of $G_{K}$, that is, the kernel of the natural surjection $G_{K} \rightarrow G_{k}$. We write Frob for the absolute (arithmetic) Frobenius elements on $k$ and on $\overline{\mathbf{F}}_{p}$, and $\mathrm{Frob}_{K}$ for the arithmetic Frobenius element of $G_{K} / I_{K} \cong G_{k}$. We 
let $\operatorname{Art}_{K}: K^{\times} \rightarrow G_{K}^{\text {ab }}$ denote the Artin map, normalized in the standard way, so the image of any uniformizer of $K$ in $G_{K} / I_{K}$ is Frob $_{K}^{-1}$.

Recall that the fundamental character $\omega_{f}: G_{K} \rightarrow k^{\times}$is defined by

$$
\omega_{f}(g)=g(\pi) / \pi \bmod \pi \mathcal{O}_{L}
$$

where $\pi$ is any root of $x^{p^{f}-1}+p=0$ and $L=K(\pi) \subset \bar{K}$. Then the composite of $\omega_{f}$ with the Artin map $K^{\times} \rightarrow G_{K}^{\mathrm{ab}} \rightarrow k^{\times}$is the homomorphism sending $p$ to 1 and any element of $\mathcal{O}_{K}^{\times}$to its reduction $\bmod p$. Replacing $\pi$ by a root of $x^{p^{f}-1}+u p=0$ for $u \in \mathcal{O}_{K}^{\times}$alters $\omega_{f}$ by an unramified character, so in fact $\left.\omega_{f}\right|_{I_{K}}$ is independent of the choice of uniformizer $u p$ of $K$. For each $\tau \in T$, we define the associated fundamental character $\omega_{\tau}: I_{K} \rightarrow \overline{\mathbf{F}}_{p}^{\times}$to be $\left.\tau \circ \omega_{f}\right|_{I_{K}}$.

A Serre weight (for $\mathrm{GL}_{2}(K)$ ) is an irreducible $\overline{\mathbf{F}}_{p}$-representation of $\mathrm{GL}_{2}(k)$. Recall that these are precisely the representations of the form

$$
V_{\vec{d}, \vec{b}}=\bigotimes_{\tau \in T}\left(\operatorname{det}^{d_{\tau}} \otimes_{k} \operatorname{Sym}^{b_{\tau}-1} k^{2}\right) \otimes_{k, \tau} \overline{\mathbf{F}}_{p}
$$

where $d_{\tau}, b_{\tau} \in \mathbf{Z}$ and $1 \leqslant b_{\tau} \leqslant p$ for each $\tau \in T$. Moreover, we can assume that $0 \leqslant d_{\tau} \leqslant p-1$ for each $\tau \in T$ and that $a_{\tau}<p-1$ for some $p$, in which case the resulting $\left(p^{f}-1\right) p^{f}$ representations $V_{\vec{d}, \vec{b}}$ are also inequivalent.

Let $\rho: G_{K} \rightarrow \mathrm{GL}_{2}\left(\overline{\mathbf{F}}_{p}\right)$ be a continuous representation. The next two subsections recall from [4] the definition of the set $W(\rho)$ of Serre weights associated to $\rho$.

\subsection{Serre weights associated to a reducible representation $\rho$. Suppose} first that $\rho$ is reducible and write $\rho \sim\left(\begin{array}{cc}x_{1} & * \\ 0 & x_{2}\end{array}\right)$. The isomorphism class of $\rho$ is then determined by the ordered pair $\left(\chi_{1}, \chi_{2}\right)$ and a cohomology class $c_{\rho} \in H^{1}\left(G_{K}, \overline{\mathbf{F}}_{p}(\chi)\right)$, where we set $\chi=\chi_{1} \chi_{2}^{-1}$. We first define a set

$$
W^{\prime}\left(\chi_{1}, \chi_{2}\right)=\left\{\left(V_{\vec{d}, \vec{b}}, J\right) \mid \begin{array}{cc}
J \subset T, & \left.\chi_{1}\right|_{I_{K}}=\prod_{\tau \in T} \omega_{\tau}^{d_{\tau}} \prod_{\tau \in J} \omega_{\tau}^{b_{\tau}} \\
\text { and } & \left.\chi_{2}\right|_{I_{K}}=\prod_{\tau \in T} \omega_{\tau}^{d_{\tau}} \prod_{\tau \notin J} \omega_{\tau}^{b_{\tau}}
\end{array}\right\} .
$$

For each pair $(V, J) \in W^{\prime}\left(\chi_{1}, \chi_{2}\right)$ we define a subspace $L_{V, J} \subset H^{1}\left(G_{K}, \overline{\mathbf{F}}_{p}(\chi)\right)$, but we first need to recall the notion of labelled Hodge-Tate weights.

Recall that if $V$ is an $n$-dimensional vector space over $\overline{\mathbf{Q}}_{p}$ and $\rho: G_{K} \rightarrow \operatorname{Aut}_{\overline{\mathbf{Q}}_{p}}(V)$ is a crystalline (hence de Rham) representation, then $D=D_{\mathrm{dR}}(V)=\left(B_{\mathrm{dR}} \otimes_{\mathbf{Q}_{p}} V\right)^{G_{K}}$ is a free module of rank $n$ over $K \otimes_{\mathbf{Q}_{p}} \overline{\mathbf{Q}}_{p}$ endowed 
with an (exhaustive, separated) decreasing filtration by (not necessarily free) $K \otimes_{\mathbf{Q}_{p}} \overline{\mathbf{Q}}_{p}$-submodules. Writing $K \otimes_{\mathbf{Q}_{p}} \overline{\mathbf{Q}}_{p} \cong \prod_{\tau \in T} \overline{\mathbf{Q}}_{p}$, we have a corresponding decomposition $D=\bigoplus_{\tau \in T} D_{\tau}$ where each $D_{\tau}$ is an $n$-dimensional filtered vector space over $\overline{\mathbf{Q}}_{p}$. For each $\tau \in T$, the multiset of $\tau$-labelled Hodge-Tate weights of $V$ are the integers $m$ with multiplicity $\operatorname{dim}_{\overline{\mathbf{Q}}_{p}} \operatorname{gr}^{-m} D_{\tau}$. In particular, if $\psi: G_{K} \rightarrow \overline{\mathbf{Q}}_{p}^{\times}$is a crystalline character, then it has a unique $\tau$-labelled Hodge-Tate weight $m_{\tau}$ for each $\tau \in T$. One finds that the vector $\vec{m}=\left(m_{\tau}\right)_{\tau \in T}$ determines $\psi$ up to an unramified character, and that $\left.\bar{\psi}\right|_{I_{K}}=\prod_{\tau \in T} \omega_{\tau}^{m_{\tau}}$.

Returning to the definition of $L_{V, J}$, suppose that $V=V_{\vec{d}, \vec{b}}$. Let $\tilde{\chi}_{1}$ be the crystalline lift of $\chi_{1}$ with $\tau$-labelled Hodge-Tate weight $d_{\tau}+b_{\tau}$ (respectively $d_{\tau}$ ) for $\tau \in J$ (respectively $\tau \notin J$ ) such that $\tilde{\chi}_{1}\left(\operatorname{Art}_{K}(p)\right)=1$, and similarly let $\tilde{\chi}_{2}$ be the crystalline lift of $\chi_{2}$ with $\tau$-labelled Hodge-Tate weight $d_{\tau}$ (respectively $d_{\tau}+b_{\tau}$ ) for $\tau \in J$ (respectively $\tau \notin J$ ) such that $\tilde{\chi}_{2}\left(\operatorname{Art}_{K}(p)\right)=1$. We then let $L_{V, J}^{\prime}$ denote the set of extension classes associated to reductions of crystalline extensions of $\tilde{\chi}_{2}$ by $\tilde{\chi}_{1}$. We then set $L_{V, J}=L_{V, J}^{\prime}$ except in the following two cases (continuing to denote $\chi_{1} \chi_{2}^{-1}$ by $\chi$ ):

- if $\chi$ is cyclotomic, $\vec{b}=(p, \ldots, p)$ and $J=T$, then $L_{V, J}=H^{1}\left(G_{K}, \overline{\mathbf{F}}_{p}(\chi)\right)$;

- if $\chi$ is trivial and $J \neq T$, then $L_{V, J}=L_{V, J}^{\prime}+H_{\mathrm{ur}}^{1}\left(G_{K}, \overline{\mathbf{F}}_{p}(\chi)\right)$ where $H_{\mathrm{ur}}^{1}\left(G_{K}, \overline{\mathbf{F}}_{p}(\chi)\right)$ is the set of unramified homomorphisms $G_{K} \rightarrow \overline{\mathbf{F}}_{p}$.

Finally we define $W(\rho)$ by the rule

$$
V \in W(\rho) \Longleftrightarrow(V, J) \in W^{\prime}\left(\chi_{1}, \chi_{2}\right) \text { and } c_{\rho} \in L_{V, J} \text { for some } J \subset T .
$$

Thus $V \in W(\rho)$ if and only if $c_{\rho} \in L_{V}$ where $L_{V}$ is defined as the union of the $L_{V, J}$ over

$$
S_{V}\left(\chi_{1}, \chi_{2}\right)=\left\{J \subset T \mid(V, J) \in W^{\prime}\left(\chi_{1}, \chi_{2}\right)\right\}
$$

(so $L_{V}$ depends on the ordered pair $\left(\chi_{1}, \chi_{2}\right)$, and it is understood to be the empty set if $\left.S_{V}\left(\chi_{1}, \chi_{2}\right)=\emptyset\right)$.

We remark that $L_{V, J}$ has dimension at least $|J|$, with equality holding unless $\chi=\chi_{1} \chi_{2}^{-1}$ is trivial or cyclotomic (see [4, Lemma 3.12]). Moreover, in a typical situation (for example if $\chi=\prod_{\tau \in T} \omega_{\tau}^{a_{\tau}}$ with $1<a_{\tau}<p-1$ for all $\tau$ ), the projection from $W^{\prime}\left(\chi_{1}, \chi_{2}\right)$ to the set of subsets of $T$ is bijective, and the projection to the set of Serre weights is injective (see [4, Section 3.2]). In that case $W^{\prime}\left(\chi_{1}, \chi_{2}\right)$ has cardinality $2^{f}$ and hence so does that of $W(\rho)$ if $c_{\rho}=0$. On the other hand, one would expect that for 'most' $\rho$, the class $c_{\rho}$ does not lie in any of the proper subspaces $L_{V, J}$ for $J \neq T$, so that $W(\rho)$ contains a single Serre weight. 
It is not however true in general that the projection from $W^{\prime}\left(\chi_{1}, \chi_{2}\right)$ to the set of Serre weights is injective, that is, $S_{V}\left(\chi_{1}, \chi_{2}\right)$ may have cardinality greater than 1 , in which case it is not immediate from the definition of $L_{V}$ that it is a subspace of $H^{1}\left(G_{K}, \overline{\mathbf{F}}_{p}(\chi)\right)$. However, it is proved in [15] if $S_{V}\left(\chi_{1}, \chi_{2}\right) \neq \emptyset$ and $p>2$, then there is an element $J_{\max } \in S_{V}\left(\chi_{1}, \chi_{2}\right)$ such that $L_{V}=L_{V, J_{\max }}$, so that $L_{V}$ is in fact a subspace. Indeed the proof of Theorem 9.1 of [15] shows that if $p>2$ and $V=V_{\vec{d}, \vec{b}}$, then $\rho$ has a crystalline lift with $\tau$-labelled Hodge-Tate weights $\left(\left\{d_{\tau}, d_{\tau}+b_{\tau}\right\}\right)_{\tau \in T}$, if and only if $S_{V}\left(\chi_{1}, \chi_{2}\right) \neq \emptyset$ and $c_{\rho} \in L_{V, J_{\max }}$. It follows that $L_{V, J} \subset L_{V, J_{\max }}$ for all $J \in S_{V}\left(\chi_{1}, \chi_{2}\right)$ (using that $J_{\max }=T$ in the exceptional case where $\chi$ is cyclotomic and $\vec{b}=(p, \ldots, p))$, and that $V \in W(\rho)$ if and only if $\rho$ has a crystalline lift with $\tau$-labelled Hodge-Tate weights $\left(\left\{d_{\tau}, d_{\tau}+b_{\tau}\right\}\right)_{\tau \in T}$.

The main aim of this paper is to use local class field theory to give a more explicit description of $H^{1}\left(G_{K}, \overline{\mathbf{F}}_{p}(\chi)\right)$, and to use this description to define subspaces which we conjecture coincide with the $L_{V}$ (even for $p=2$ ).

\subsection{Serre weights associated to an irreducible representation $\rho$. While the} focus of this paper is on the case where $\rho$ is reducible, for completeness we recall the definition of $W(\rho)$ in the case where $\rho$ is irreducible. We let $K^{\prime}$ denote the quadratic unramified extension of $K, k^{\prime}$ the residue field of $K^{\prime}, T^{\prime}$ the set of embeddings of $k^{\prime}$ in $\overline{\mathbf{F}}_{p}$, and $\pi$ the natural projection $T^{\prime} \rightarrow T$. For $\tau^{\prime} \in T^{\prime}$, we let $\omega_{\tau^{\prime}}$ denote the corresponding fundamental character of $I_{K^{\prime}}=I_{K}$. Note that if $\rho$ is irreducible, then it is necessarily tamely ramified and in fact induced from a character of $G_{K^{\prime}}$. We define $W(\rho)$ by the rule:

$$
\begin{aligned}
& \left.\rho\right|_{I} \sim \prod_{\tau \in T} \omega_{\tau}^{d_{\tau}}\left(\begin{array}{cc}
\prod_{\tau^{\prime} \in J^{\prime}} \omega_{\tau^{\prime}}^{b_{\pi\left(\tau^{\prime}\right)}} & 0 \\
0 & \prod_{\tau^{\prime} \notin J^{\prime}} \omega_{\tau^{\prime}}^{b_{\pi\left(\tau^{\prime}\right)}}
\end{array}\right) \\
& V_{\vec{d}, \vec{b}} \in W(\rho) \Longleftrightarrow
\end{aligned}
$$

for some $J^{\prime} \subset T^{\prime}$ such that $\pi: J^{\prime} \stackrel{\sim}{\longrightarrow} T$.

It is true in this case as well that $W(\rho)$ typically has cardinality $2^{f}$ (see $[4$, Section 3.1]). Moreover, the result of [15] characterizing $W(\rho)$ in terms of reductions of crystalline representations (for $p>2$ ) holds in the irreducible case as well.

2.4. The case $\boldsymbol{K}=\mathbf{Q}_{\boldsymbol{p}}$. To indicate the level of complexity hidden in the general recipe for weights, we describe the set $W(\rho)$ more explicitly in the classical case $K=\mathbf{Q}_{p}$. Replacing $\rho$ by a twist, we can assume $\left.\rho\right|_{I_{\mathbf{Q}_{p}}}$ has the form $\left(\begin{array}{cc}\omega_{2}^{a} & 0 \\ 0 & \omega_{2}^{p a}\end{array}\right)$ or $\left(\begin{array}{cc}\omega^{a} & * \\ 0 & 1\end{array}\right)$ for some $a$ with $1 \leqslant a \leqslant p-1$ (where $\omega=\omega_{1}$ is the mod 
$p$ cyclotomic character). In the first case we find that $W(\rho)=\left\{V_{0, a}, V_{a-1, p+1-a}\right\}$ (with the two weights coinciding if $a=1$ ). In the second case we may further assume (after twisting) that $\rho=\left(\begin{array}{cc}\chi & * \\ 0 & 1\end{array}\right)$ for some character $\chi: G_{\mathbf{Q}_{p}} \rightarrow \overline{\mathbf{F}}_{p}^{\times}$. Since the space $H^{1}\left(G_{\mathbf{Q}_{p}}, \overline{\mathbf{F}}_{p}(\chi)\right)$ is one-dimensional unless $\chi$ is trivial or cyclotomic, one does not need much information about the spaces $L_{V, J}$ in order to determine $W(\rho)$; indeed all one needs is that:

- $L_{V, T}=H^{1}\left(G_{\mathbf{Q}_{p}}, \overline{\mathbf{F}}_{p}(\chi)\right)$ unless $\chi$ is cyclotomic and $V=V_{0,1}$;

- $L_{V_{0,1}, T}$ is the peu ramifiée subspace if $\chi$ is cyclotomic, that is, the subspace corresponding to $\mathbf{Z}_{p}^{\times} \otimes \overline{\mathbf{F}}_{p}^{\times}$under the Kummer isomorphism $H^{1}\left(G_{\mathbf{Q}_{p}}, \mu_{p}\right) \cong$ $\mathbf{Q}_{p}^{\times} /\left(\mathbf{Q}_{p}^{\times}\right)^{p}$.

- $L_{V, \emptyset}=0$ if $\chi \neq 1$.

It then follows (see [4]) that

$$
W(\rho)= \begin{cases}\left\{V_{0, a}\right\} & \text { if } 1<a<p-1 \text { and } \rho \text { is nonsplit, } \\ \left\{V_{0, a}, V_{a, p-1-a}\right\} & \text { if } 1<a<p-2 \text { and } \rho \text { is split, } \\ \left\{V_{0, p-2}, V_{p-2, p}, V_{p-2,1}\right\} & \text { if } a=p-2, p>3 \text { and } \rho \text { is split, } \\ \left\{V_{0, p-1}\right\} & \text { if } a=p-1 \text { and } p>2, \\ \left\{V_{0, p}\right\} & \text { if } a=1, \chi=\omega \text { and } \rho \text { is not peu ramifiée, } \\ \left\{V_{0, p}, V_{0,1}, V_{1, p-2}\right\} & \text { if } a=1, p>3 \text { and } \rho \text { is split, } \\ \left\{V_{0,3}, V_{0,1}, V_{1,3}, V_{1,1}\right\} & \text { if } a=1, p=3 \text { and } \rho \text { is split, } \\ \left\{V_{0, p}, V_{0,1}\right\} & \text { otherwise. }\end{cases}
$$

We remark that the first case above is the most typical and the next one arises in the setting of 'companion forms'. The remaining cases take into account special situations that arise when $\left.\chi\right|_{I_{K}}$ or its inverse is trivial or cyclotomic.

2.5. Serre's conjecture over totally real fields. We now recall how Serre weights arise in the context of Galois representations associated to automorphic forms. Let $F$ be a totally real field in which $p$ is unramified. Let $\mathcal{O}_{F}$ denote its ring of integers and $S_{p}$ the set of primes of $\mathcal{O}_{F}$ dividing $p$. For each $\mathfrak{p} \in S_{p}$, we let $k_{\mathfrak{p}}=\mathcal{O}_{F} / \mathfrak{p}, f_{\mathfrak{p}}=\left[k_{\mathfrak{p}}: \mathbf{F}_{p}\right]$ and $T_{\mathfrak{p}}$ the set of embeddings $\tau: k_{\mathfrak{p}} \rightarrow \overline{\mathbf{F}}_{p}$. The irreducible $\overline{\mathbf{F}}_{p}$-representations of $\mathrm{GL}_{2}(\mathcal{O} / p \mathcal{O}) \cong \prod_{\mathfrak{p} \in S_{\mathfrak{p}}} \mathrm{GL}_{2}\left(k_{\mathfrak{p}}\right)$ are then of the form: $V=\bigotimes_{\left\{\mathfrak{p} \in S_{p}\right\}} V_{\mathfrak{p}}$ where each $V_{\mathfrak{p}}$ is a Serre weight for $\mathrm{GL}_{2}\left(F_{\mathfrak{p}}\right)$.

Suppose that $\rho: G_{F} \rightarrow \mathrm{GL}_{2}\left(\overline{\mathbf{F}}_{p}\right)$ is continuous, irreducible and totally odd. A notion of $\rho$ being modular of weight $V$ is introduced in [4], where the following generalization of Serre's Conjecture (from [24]) is made: 
CONJeCtURe 2.1. The representation $\rho$ is modular of weight $V=\bigotimes_{\left\{\mathfrak{p} \in S_{p}\right\}} V_{\mathfrak{p}}$ if and only if $V_{\mathfrak{p}} \in W\left(\left.\rho\right|_{G_{F_{\mathfrak{p}}}}\right)$ for all $\mathfrak{p} \in S_{p}$.

We refer the reader to [4] for the definition of modularity of weight $V$ and its relation to the usual notion of weights of Hilbert modular forms. We just remark that $\rho$ is modular of some weight $V$ if and only if $\rho$ is modular in the usual sense that $\rho \cong \bar{\rho}_{f}$ for some Hilbert modular eigenform $f$, and that the set of weights for which $\rho$ is modular determines the possible cohomological weights and local behaviour at primes over $p$ of those eigenforms (see [4, Proposition 2.10]).

Under the assumption that $\rho$ is modular (of some weight), Conjecture 2.1 can be viewed as the generalization of the weight part of Serre's Conjecture and has been proved under mild technical hypotheses (for $p>2$ ) in a series of papers by Gee and co-authors culminating in [15], together with the results of either Gee and Kisin [14] or Newton [20]. Moreover, their result holds without the assumption that $p$ is unramified in $F$ using the description of $W(\rho)$ in terms of reductions of crystalline representations.

Finally we remark that Conjecture 2.1 is known in the case $F=\mathbf{Q}$. In this case the modularity of $\rho$ is a theorem of Khare and Wintenberger [17, 18], and the weight part follows from prior work of Gross, Edixhoven and others (see [4, Theorem 3.20]); it amounts to the statement that if $2 \leqslant k \leqslant p+1$, then $\omega^{d} \rho$ arises from a Hecke eigenform of weight $k$ and level prime to $p$ if and only if $V_{d, k-1} \in W(\rho)$.

\section{The ramification filtration on cohomology}

In this section we use the upper numbering of ramification groups to define filtrations on the Galois cohomology groups parametrizing the extensions of characters under consideration.

3.1. Definition of the filtration. Continue to let $K$ denote a finite unramified extension of $\mathbf{Q}_{p}$ of degree $f$ with residue field $k$, and let $\chi: G_{K} \rightarrow \overline{\mathbf{F}}_{p}^{\times}$be any character. Recall from [23, IV.3] that $G_{K}$ has a decreasing filtration by closed subgroups $G_{K}^{u}$ where $G_{K}^{-1}=G_{K}, G_{K}^{u}=I_{K}$ for $-1<u \leqslant 0$, and $\bigcup_{u>0} G_{K}^{u}$ is the wild ramification subgroup $P_{K}$. We define an increasing filtration on $H^{1}\left(G_{K}, \overline{\mathbf{F}}_{p}(\chi)\right)$ by setting

$$
\operatorname{Fil}^{s} H^{1}\left(G_{K}, \overline{\mathbf{F}}_{p}(\chi)\right)=\bigcap_{u>s-1} \operatorname{ker}\left(H^{1}\left(G_{K}, \overline{\mathbf{F}}_{p}(\chi)\right) \rightarrow H^{1}\left(G_{K}^{u}, \overline{\mathbf{F}}_{p}(\chi)\right)\right)
$$

for $s \in \mathbf{R}$. Note that $\mathrm{Fil}^{s} H^{1}\left(G_{K}, \overline{\mathbf{F}}_{p}(\chi)\right)=0$ for $s<0$, and that

$$
\operatorname{Fil}^{0} H^{1}\left(G_{K}, \overline{\mathbf{F}}_{p}(\chi)\right)=\operatorname{ker}\left(H^{1}\left(G_{K}, \overline{\mathbf{F}}_{p}(\chi)\right) \rightarrow H^{1}\left(I_{K}, \overline{\mathbf{F}}_{p}(\chi)\right)\right) .
$$


Let $z$ be a cocycle representing a class in $c \in H^{1}\left(G_{K}, \overline{\mathbf{F}}_{p}(\chi)\right)$. Since $\left.\chi\right|_{P_{K}}$ is trivial, the restriction of $z$ defines a homomorphism $P_{K} \rightarrow \overline{\mathbf{F}}_{p}$; so if $s \geqslant 1$, then $c \in \operatorname{Fil}^{s}\left(H^{1}\left(G_{K}, \overline{\mathbf{F}}_{p}(\chi)\right)\right)$ if and only if $z\left(G_{K}^{u}\right)=0$ for all $u>s-1$. In particular, $c \in \operatorname{Fil}^{1}\left(H^{1}\left(G_{K}, \overline{\mathbf{F}}_{p}(\chi)\right)\right)$ if and only if $z\left(P_{K}\right)=0$; since $H^{1}\left(I_{K} / P_{K}, \overline{\mathbf{F}}_{p}(\chi)\right)=0$, it follows that $\operatorname{Fil}^{s} H^{1}\left(G_{K}, \overline{\mathbf{F}}_{p}(\chi)\right)=\operatorname{Fil}^{0} H^{1}\left(G_{K}, \overline{\mathbf{F}}_{p}(\chi)\right)$ for $0 \leqslant s \leqslant 1$.

3.2. Computation of the jumps in the filtration. For any $s \in \mathbf{R}$, we set $\operatorname{Fil}^{<s}\left(H^{1}\left(G_{K}, \overline{\mathbf{F}}_{p}(\chi)\right)\right)=\bigcup_{t<s} \operatorname{Fil}^{t}\left(H^{1}\left(G_{K}, \overline{\mathbf{F}}_{p}(\chi)\right)\right)$. Since $G_{K}^{u}=\bigcap_{v<u} G_{K}^{v}$, the compactness of $G_{K}$ and continuity of the cocycle $z$ imply that in fact

$$
\operatorname{Fil}^{<s}\left(H^{1}\left(G_{K}, \overline{\mathbf{F}}_{p}(\chi)\right)\right)=\operatorname{ker}\left(H^{1}\left(G_{K}, \overline{\mathbf{F}}_{p}(\chi)\right) \rightarrow H^{1}\left(G_{K}^{s-1}, \overline{\mathbf{F}}_{p}(\chi)\right)\right) .
$$

We now compute the jumps in the filtration, that is, the dimension of

$$
\operatorname{gr}^{s}\left(H^{1}\left(G_{K}, \overline{\mathbf{F}}_{p}(\chi)\right)\right)=\operatorname{Fil}^{s}\left(H^{1}\left(G_{K}, \overline{\mathbf{F}}_{p}(\chi)\right)\right) / \mathrm{Fil}^{<s}\left(H^{1}\left(G_{K}, \overline{\mathbf{F}}_{p}(\chi)\right)\right)
$$

for every $s$ and $\chi$.

We must first introduce some notation. Choose an embedding $\tau_{0}: k \rightarrow \overline{\mathbf{F}}_{p}$, let $\tau_{i}=\tau_{0} \circ$ Frob $^{i}$ where Frob is the absolute Frobenius on $k$. Recall that $\omega_{f}: G_{K} \rightarrow k^{\times}$denotes the character defined by

$$
\omega_{f}(g)=g(\pi) / \pi,
$$

where $\pi$ is any root of $x^{p^{f}-1}=-p$ in $\bar{K}$, and set $\omega_{f, i}=\omega_{\tau_{i}}=\tau_{i} \circ \omega_{f}$ for $i=0$, $\ldots, f-1$. We may then write $\left.\chi\right|_{I_{K}}=\left.\omega_{f, 0}^{n}\right|_{I_{K}}$ where $n=\sum_{j=0}^{f-1} a_{j} p^{j}$ for integers $a_{j}$ satisfying $1 \leqslant a_{j} \leqslant p$ for $j=0, \ldots, f-1$. Moreover, this expression is unique if we further require (in the case that $\left.\chi\right|_{I_{K}}$ is the cyclotomic character) that some $a_{j} \neq p$ for some $j$. We extend the definition of $a_{j}$ to all integers $j$ by setting $a_{j}=a_{j^{\prime}}$ if $j \equiv j^{\prime} \bmod f$. We define $\left(a_{0}, a_{1}, \ldots, a_{f-1}\right)$ to be the tame signature of $\chi$; thus the tame signature of $\chi$ is an element of the set

$$
S=\{1,2, \ldots, p\}^{f}-\{(p, p, \ldots, p)\} .
$$

Define an action of $\operatorname{Gal}\left(k / \mathbf{F}_{p}\right)=\langle$ Frob $\rangle \cong \mathbf{Z} / f \mathbf{Z}$ on $S$ by the formula

$$
\text { Frob } \cdot\left(a_{0}, a_{1}, \ldots, a_{f-1}\right)=\left(a_{f-1}, a_{0}, \ldots, a_{f-2}\right) \text {. }
$$

Note that if $\chi$ has tame signature $\vec{a}$, then $\operatorname{Frob} \circ \chi$ has tame signature $\operatorname{Frob}(\vec{a})$, as does $\chi \circ \sigma$ where $\sigma$ is the (outer) automorphism of $G_{K}$ defined by conjugation by a lift of Frob $\in \operatorname{Gal}\left(k / \mathbf{F}_{p}\right) \cong \operatorname{Gal}\left(K / \mathbf{Q}_{p}\right)$ to $G_{\mathbf{Q}_{p}}$. We define be the period of $\vec{a} \in S$ to be the cardinality of its orbit under $\operatorname{Gal}\left(k / \mathbf{F}_{p}\right)$, and the absolute niveau of $\chi$ to be the period of its tame signature. (Note that the orbit of the tame signature of $\chi$ under $\operatorname{Gal}\left(k / \mathbf{F}_{p}\right)$ is independent of the choice of $\tau_{0}$.) 
For $i=0, \ldots, f-1$, we define

$$
n_{i}=\sum_{j=0}^{f-1} a_{i+j} p^{j}
$$

so that $n_{0} \equiv n_{i} p^{i} \bmod \left(p^{f}-1\right)$ and $\left.\chi\right|_{I_{K}}=\left.\omega_{f, i}^{n_{i}}\right|_{I_{K}}$.

THEOREM 3.1. Let $d_{s}=\operatorname{dim}_{\overline{\mathbf{F}}_{p}} \operatorname{gr}^{s}\left(H^{1}\left(G_{K}, \overline{\mathbf{F}}_{p}(\chi)\right)\right)$ for $s \in \mathbf{R}$. Then $d_{s}=0$ unless $s=0$ or $1<s \leqslant 1+p /(p-1)$. Moreover, if $d_{s} \neq 0$ and $1<s<$ $1+p /(p-1)$, then $s=1+m /\left(p^{f}-1\right)$ for some integer $m$ not divisible by $p$. More precisely, if $\chi$ has tame signature $\left(a_{0}, a_{1}, \ldots, a_{f-1}\right)$ of period $f^{\prime}$ and the integers $n_{i}$ are defined by (5), then:

(1) $d_{0}=1$ if $\chi$ is trivial and $d_{0}=0$ otherwise;

(2) if $1<s<p /(p-1)$, then

$$
d_{s}=\left\{\begin{array}{l}
\quad \begin{array}{l}
\text { if } s=n_{i+k} /\left(p^{f}-1\right) \text { for some } i, k \text { such that } k>0, \\
f / f^{\prime} \quad a_{i}=p, a_{i+1}=\cdots=a_{i+k-1}=p-1 \text { and } a_{i+k} \neq \\
p-1,
\end{array} \\
\quad \text { otherwise; }
\end{array}\right.
$$

(3) if $p /(p-1) \leqslant s<1+p /(p-1)$, then

$$
d_{s}= \begin{cases}f / f^{\prime} & \text { if } s=1+n_{i} /\left(p^{f}-1\right) \text { for some } i \text { such that } a_{i} \neq p, \\ 0 & \text { otherwise }\end{cases}
$$

(4) $d_{1+p /(p-1)}=1$ if $\chi$ is cyclotomic, and $d_{1+p /(p-1)}=0$ otherwise.

Proof. We let $d_{s}^{\prime}$ denote the value claimed for $d_{s}$ in the statement. Note that if $1<s<p /(p-1)$, then $d_{s}^{\prime}$ is the number of $j \in R$ such that $s=n_{j} /\left(p^{f}-1\right)$, where $R$ is the set of $j \in\{0, \ldots, f-1\}$ such that $a_{j} \neq p-1$ and $\left(a_{i}, a_{i+1}, \ldots\right.$, $\left.a_{j-1}\right)=(p, p-1, \ldots, p-1)$ for some $i$ with $j-f \leqslant i<j$. Moreover, $R$ is in bijection with the set of $i \in\{0, \ldots, f-1\}$ such that $a_{i}=p$, and if $j \in R$, then $1<n_{i} /\left(p^{f}-1\right)<p /(p-1)$. Therefore,

$$
\sum_{1<s<p /(p-1)} d_{s}^{\prime}=\#\left\{i \in\{0, \ldots, f-1\} \mid a_{i}=p\right\} .
$$

Similarly if $p /(p-1) \leqslant s<1+p /(p-1)$, then $d_{s}^{\prime}$ is the number of $i \in\{0, \ldots, f-1\}$ such that $s=1+n_{i} /\left(p^{f}-1\right)$ and $a_{i} \neq p$; moreover, if 
$a_{i} \neq p$, then $p /(p-1) \leqslant 1+n_{i} /\left(p^{f}-1\right)<1+p /(p-1)$, so

$$
\sum_{p /(p-1) \leqslant s<1+p /(p-1)} d_{s}^{\prime}=\#\left\{i \in\{0, \ldots, f-1\} \mid a_{i} \neq p\right\} .
$$

It follows that

$$
\sum_{s \in \mathbf{R}} d_{s}^{\prime}= \begin{cases}f+2 & \text { if } p=2 \text { and } \chi \text { is trivial } \\ f+1 & \text { if } p>2 \text { and } \chi \text { is trivial or cyclotomic } \\ f & \text { otherwise }\end{cases}
$$

Therefore, $\sum_{s \in \mathbf{R}} d_{s}^{\prime}=\operatorname{dim}_{\overline{\mathbf{F}}_{p}} H^{1}\left(G_{K}, \overline{\mathbf{F}}_{p}(\chi)\right)=\sum_{s \in \mathbf{R}} d_{s}$, so it suffices to prove that $d_{s}^{\prime} \leqslant d_{s}$ for all $s$, and we need only consider $s$ such that $d_{s}^{\prime}>0$.

For $s=0$, the inflation-restriction exact sequence

$$
0 \rightarrow H^{1}\left(G_{K} / I_{K}, \overline{\mathbf{F}}_{p}(\chi)^{I_{K}}\right) \rightarrow H^{1}\left(G_{K}, \overline{\mathbf{F}}_{p}(\chi)\right) \rightarrow H^{1}\left(I_{K}, \overline{\mathbf{F}}_{p}(\chi)\right)
$$

shows that $\operatorname{gr}^{0} H^{1}\left(G_{K}, \overline{\mathbf{F}}_{p}(\chi)\right) \cong H^{1}\left(G_{K} / I_{K}, \overline{\mathbf{F}}_{p}(\chi)^{I_{K}}\right)$ has dimension 1 if $\chi$ is trivial, and 0 otherwise, so that $d_{0}=d_{0}^{\prime}$. We may therefore assume that $s>1$ and that $m=(s-1)\left(p^{f}-1\right)$ is an integer. Moreover, either $0<m<$ $p\left(p^{f}-1\right) /(p-1)$ and $m$ is not divisible by $p$, or $m=p\left(p^{f}-1\right) /(p-1)$.

Let $M=L(\pi)$ where $\pi^{p^{f}-1}=-p$ and $L$ is an unramified extension of $K$ of degree prime to $p$ such that $\left.\chi\right|_{G_{M}}$ is trivial; thus $\chi=\mu \omega_{f, 0}^{n_{0}}$ for some unramified character $\mu$ of $\operatorname{Gal}(L / K)$. Since $\operatorname{Gal}(M / K)$ has order prime to $p$, inflationrestriction gives

$$
H^{1}\left(G_{K}, \overline{\mathbf{F}}_{p}(\chi)\right) \cong H^{1}\left(G_{M}, \overline{\mathbf{F}}_{p}(\chi)\right)^{\mathrm{Gal}(M / K)}=\operatorname{Hom}_{\mathrm{Gal}(M / K)}\left(G_{M}^{\mathrm{ab}}, \overline{\mathbf{F}}_{p}(\chi)\right),
$$

which we identify with

$$
\operatorname{Hom}_{\mathrm{Gal}(M / K)}\left(M^{\times}, \overline{\mathbf{F}}_{p}(\chi)\right)=\operatorname{Hom}_{\mathrm{Gal}(M / K)}\left(M^{\times} /\left(M^{\times}\right)^{p}, \overline{\mathbf{F}}_{p}(\chi)\right)
$$

via the isomorphism $M^{\times} \cong W_{M}^{\mathrm{ab}} \subset G_{M}^{\mathrm{ab}}$ of local class field theory.

Since $M$ is tamely ramified over $K$, we have $G_{K}^{u} \subset G_{M}$ for $u>0$, and in fact $G_{K}^{u}=G_{M}^{u\left(p^{f}-1\right)}$ by [23, IV, Proposition 15], which maps onto $1+\pi^{\left\lceil u\left(p^{f}-1\right)\right\rceil} \mathcal{O}_{M}$ under the homomorphism $W_{M} \rightarrow M^{\times}$of local class field theory (see [23, Corollary 3 to Theorem 1]). Therefore, a class in $H^{1}\left(G_{K}\right.$, $\overline{\mathbf{F}}_{p}(\chi)$ ) has trivial restriction to $G_{K}^{u}$ for all $u>s-1$ (respectively $G_{K}^{s-1}$ ) if and only if the corresponding homomorphism $M^{\times} /\left(M^{\times}\right)^{p} \rightarrow \overline{\mathbf{F}}_{p}(\chi)$ factors through $M^{\times} /\left(M^{\times}\right)^{p} U_{m+1}$ (respectively $\left.M^{\times} /\left(M^{\times}\right)^{p} U_{m}\right)$, where we write $U_{t}=1+\pi^{t} \mathcal{O}_{M}$ for a positive integer $t$. It follows that

$$
\operatorname{gr}^{s}\left(H^{1}\left(G_{K}, \overline{\mathbf{F}}_{p}(\chi)\right)\right) \cong \operatorname{Hom}_{\mathrm{Gal}(M / K)}\left(U_{m} /\left(U_{m} \cap\left(M^{\times}\right)^{p}\right) U_{m+1}, \overline{\mathbf{F}}_{p}(\chi)\right) .
$$


Now suppose that $m<p\left(p^{f}-1\right) /(p-1)$ and $m$ is not divisible by $p$. Then we claim that $U_{m} \cap\left(M^{\times}\right)^{p} \subset U_{m+1}$. Indeed suppose that $v_{\pi}\left(x^{p}-1\right)=m$ for some $x \in M^{\times}$, and let $t=v_{\pi}(x-1)$. Then $t>0$ and writing $x=1+y \pi^{t}$ for some $y \in \mathcal{O}_{M}^{\times}$, we have

$$
x^{p}-1=\left(1+y \pi^{t}\right)^{p}-1=p y \pi^{t}+\cdots+y^{p} \pi^{p t} .
$$

So $m \geqslant \min \left(t+p^{f}-1, t p\right)$, with equality unless $t+p^{f}-1=t p$. If $t+p^{f}-1>t p$, then $m=t p$ contradicts that $m$ is not divisible by $p$, and if $t+p^{f}-1 \leqslant t p$, then $t \geqslant p^{f}-1 /(p-1)$ contradicts that $m<p\left(p^{f}-1\right) /(p-1)$. This establishes the claim, from which it follows that

$$
\operatorname{gr}^{s} H^{1}\left(G_{K}, \overline{\mathbf{F}}_{p}(\chi)\right) \cong \operatorname{Hom}_{\mathrm{Gal}(M / K)}\left(U_{m} / U_{m+1}, \overline{\mathbf{F}}_{p}(\chi)\right)
$$

Letting $l$ denote the residue field of $L$, the map $x \mapsto 1+x \pi^{m}$ induces a $\operatorname{Gal}(M / K)$ equivariant isomorphism $l\left(\omega_{f}^{m}\right) \cong U_{m} / U_{m+1}$, and the map $x \otimes 1 \mapsto(\sigma(x))_{\sigma}$ induces a $\operatorname{Gal}(M / K)$-equivariant isomorphism

$$
l\left(\omega_{f}^{m}\right) \otimes_{\mathbf{F}_{p}} \overline{\mathbf{F}}_{p} \cong \bigoplus_{i=0}^{f-1}\left(\bigoplus_{\sigma \in S_{i}} \overline{\mathbf{F}}_{p}\left(\omega_{f, i}^{m}\right)\right)
$$

where $S_{i}$ is the set of embeddings $l \rightarrow \overline{\mathbf{F}}_{p}$ restricting to $\tau_{i}$ and the action of $\operatorname{Gal}(M / K)$ on $\bigoplus_{\sigma \in S_{i}} \overline{\mathbf{F}}_{p}\left(\omega_{f, i}^{m}\right)$ is defined by $g\left(\left(x_{\sigma}\right)_{\sigma}\right)=\omega_{f, i}^{m}(g)\left(x_{\sigma \circ g}\right)_{\sigma}$. Noting that $\bigoplus_{\sigma \in S_{i}} \overline{\mathbf{F}}_{p} \cong \operatorname{Ind}_{\mathrm{Gal}(M / L)}^{\mathrm{Gal}(M / K)} \overline{\mathbf{F}}_{p}$, we see that

$$
\left(U_{m} / U_{m+1}\right) \otimes_{\mathbf{F}_{p}} \overline{\mathbf{F}}_{p} \cong \bigoplus_{i=0}^{f-1} \bigoplus_{\mu} \overline{\mathbf{F}}_{p}\left(\mu \omega_{f, i}^{m}\right),
$$

where the second direct sum is over all characters $\mu: \operatorname{Gal}(L / K) \rightarrow \overline{\mathbf{F}}_{p}^{\times}$. Therefore, $d_{s}$ is the number of $i$ such that $m \equiv n_{i} \bmod \left(p^{f}-1\right)$. The inequality $d_{s}^{\prime} \leqslant d_{s}$ is now immediate from the definition of $d_{s}^{\prime}$.

Finally consider the case $s=1+p /(p-1)$, so $m=p\left(p^{f}-1\right) /(p-1)$; we may assume $\chi$ is cyclotomic, and it suffices to prove that $d_{s} \geqslant 1$. For $x \in U_{m+1}$, we see that $\exp \left(p^{-1} \log x\right)$ converges to a $p$ th root of $x$, so $U_{m+1} \subset\left(M^{\times}\right)^{p}$. It follows that $\mathrm{Fil}^{s} H^{1}\left(G_{K}, \overline{\mathbf{F}}_{p}(\chi)\right)=H^{1}\left(G_{K}, \overline{\mathbf{F}}_{p}(\chi)\right)$. Therefore, it suffices to prove that $\mathrm{Fil}^{<s} H^{1}\left(G_{K}, \overline{\mathbf{F}}_{p}(\chi)\right) \neq H^{1}\left(G_{K}, \overline{\mathbf{F}}_{p}(\chi)\right)$, that is, that there is a class in $H^{1}\left(G_{K}, \overline{\mathbf{F}}_{p}(\chi)\right)$ whose restriction to $G_{K}^{p /(p-1)}$ is nontrivial. Since 
$G_{K}^{p /(p-1)}=G_{\mathbf{Q}_{p}}^{p /(p-1)}$, the diagram

$$
\begin{array}{r}
H^{1}\left(G_{\mathbf{Q}_{p}}, \overline{\mathbf{F}}_{p}(\chi)\right) \\
\downarrow \\
\downarrow \\
H^{1}\left(G_{K}, H^{1}\left(G_{\mathbf{Q}_{p}}^{p /(p-1)}, \overline{\mathbf{F}}_{p}(\chi)\right)\right. \\
\| H^{1}\left(G_{K}^{p /(p-1)}, \overline{\mathbf{F}}_{p}(\chi)\right)
\end{array}
$$

reduces us to the case $K=\mathbf{Q}_{p}$, and we may further assume $M=\mathbf{Q}_{p}(\pi)=$ $\mathbf{Q}_{p}\left(\zeta_{p}\right)$. We see in this case that if $x \in U_{1}$, then $x^{p} \in U_{p+1}$, so that $U_{p} \cap\left(M^{\times}\right)^{p} \subset$ $U_{p+1}$ (and in fact equality holds). It follows that

$$
\operatorname{gr}^{s}\left(H^{1}\left(G_{\mathbf{Q}_{p}}, \overline{\mathbf{F}}_{p}(\chi)\right)\right) \cong \operatorname{Hom}_{\mathrm{Gal}\left(M / \mathbf{Q}_{p}\right)}\left(U_{p} / U_{p+1}, \mathbf{F}_{p}(\chi)\right),
$$

which is nontrivial (in fact one-dimensional) since $U_{p} / U_{p+1} \cong \mathbf{F}_{p}(\chi)$.

3.3. Terminology associated with ramification. Note that the dimensions $d_{s}$ in Theorem 3.1 are at most 1 if $\chi$ has absolute niveau $f$, in which case we say $\chi$ is primitive; otherwise we say $\chi$ is imprimitive. Thus $\chi$ is imprimitive if and only if its tame signature $\left(a_{0}, a_{1}, \ldots, a_{f-1}\right)$ has nontrivial rotational symmetry, which is equivalent to $\chi$ extending to a character of $G_{K^{\prime}}$ for some proper subfield $K^{\prime}$ of $K$ containing $\overline{\mathbf{Q}}_{p}$.

The statement of the theorem is also simpler if $a_{i}<p$ for all $i$, in which case we say $\chi$ is generic; otherwise we say $\chi$ is nongeneric. Thus if $\chi$ is generic, then $d_{s}=$ 0 if $1<s<p /(p-1)$ (by part (2) of the theorem); moreover, $n_{i} \leqslant p^{f}-1$ for all $i$, so we also have $d_{s}=0$ if $2<s<1+p /(p-1)$ (by part (3) of the theorem). To characterize the types of exceptional behaviour arising in extensions when $\chi$ is nongeneric (or trivial or cyclotomic), we introduce the following subspaces of $H^{1}\left(G_{K}, \overline{\mathbf{F}}_{p}(\chi)\right)$ :

$$
\begin{aligned}
H_{\mathrm{un}}^{1}\left(G_{K}, \overline{\mathbf{F}}_{p}(\chi)\right) & =\mathrm{Fil}^{0} H^{1}\left(G_{K}, \overline{\mathbf{F}}_{p}(\chi)\right)=\mathrm{Fil}^{1} H^{1}\left(G_{K}, \overline{\mathbf{F}}_{p}(\chi)\right) ; \\
H_{\mathrm{gt}}^{1}\left(G_{K}, \overline{\mathbf{F}}_{p}(\chi)\right) & =\mathrm{Fil}^{<p /(p-1)} H^{1}\left(G_{K}, \overline{\mathbf{F}}_{p}(\chi)\right) ; \\
H_{\mathrm{fl}}^{1}\left(G_{K}, \overline{\mathbf{F}}_{p}(\chi)\right) & =\mathrm{Fil}^{p /(p-1)} H^{1}\left(G_{K}, \overline{\mathbf{F}}_{p}(\chi)\right) ; \\
H_{\mathrm{cg}}^{1}\left(G_{K}, \overline{\mathbf{F}}_{p}(\chi)\right) & =\mathrm{Fil}^{2} H^{1}\left(G_{K}, \overline{\mathbf{F}}_{p}(\chi)\right) ; \\
H_{\mathrm{ty}}^{1}\left(G_{K}, \overline{\mathbf{F}}_{p}(\chi)\right) & =\mathrm{Fil}^{<1+p /(p-1)} H^{1}\left(G_{K}, \overline{\mathbf{F}}_{p}(\chi)\right) .
\end{aligned}
$$

We call $H_{\text {un }}^{1}\left(G_{K}, \overline{\mathbf{F}}_{p}(\chi)\right)$ the unramified subspace of $H^{1}\left(G_{K}, \overline{\mathbf{F}}_{p}(\chi)\right)$, and we call $H_{\mathrm{gt}}^{1}\left(G_{K}, \overline{\mathbf{F}}_{p}(\chi)\right)$ (respectively $H_{\mathrm{fl}}^{1}\left(G_{K}, \overline{\mathbf{F}}_{p}(\chi)\right), \quad H_{\mathrm{cg}}^{1}\left(G_{K}, \overline{\mathbf{F}}_{p}(\chi)\right)$, $\left.H_{\mathrm{ty}}^{1}\left(G_{K}, \overline{\mathbf{F}}_{p}(\chi)\right)\right)$ the gently (respectively flatly, cogently, typically) ramified subspace of $H^{1}\left(G_{K}, \overline{\mathbf{F}}_{p}(\chi)\right)$. We use the same terminology to describe the cohomology classes in these subspaces.

The following is immediate from Theorem 3.1: 
COROLLARY 3.2. With the above notation, we have

(1) $H_{\mathrm{un}}^{1}\left(G_{K}, \overline{\mathbf{F}}_{p}(\chi)\right)=0$ unless $\chi$ is trivial, in which case $H_{\mathrm{un}}^{1}\left(G_{K}, \overline{\mathbf{F}}_{p}(\chi)\right)$ has dimension 1;

(2) $H^{1}\left(G_{K}, \overline{\mathbf{F}}_{p}(\chi)\right) / H_{\mathrm{ty}}^{1}\left(G_{K}, \overline{\mathbf{F}}_{p}(\chi)\right)=0$ unless $\chi$ is cyclotomic, in which case it has dimension 1 ;

(3) $H_{\mathrm{fl}}^{1}\left(G_{K}, \overline{\mathbf{F}}_{p}(\chi)\right) / H_{\mathrm{gt}}^{1}\left(G_{K}, \overline{\mathbf{F}}_{p}(\chi)\right)=0$ unless $\left.\chi\right|_{I_{K}}$ is cyclotomic, in which case it has dimension $f$;

(4) $H_{\mathrm{ty}}^{1}\left(G_{K}, \overline{\mathbf{F}}_{p}(\chi)\right) / H_{\mathrm{un}}^{1}\left(G_{K}, \overline{\mathbf{F}}_{p}(\chi)\right)$ has dimension $f$;

(5) $H_{\mathrm{gt}}^{1}\left(G_{K}, \overline{\mathbf{F}}_{p}(\chi)\right) / H_{\mathrm{un}}^{1}\left(G_{K}, \overline{\mathbf{F}}_{p}(\chi)\right)$ has dimension equal to the number of $i \in$ $\{0, \ldots, f-1\}$ such that $a_{i}=p$;

(6) $H_{\mathrm{cg}}^{1}\left(G_{K}, \overline{\mathbf{F}}_{p}(\chi)\right)=H_{\mathrm{ty}}^{1}\left(G_{K}, \overline{\mathbf{F}}_{p}(\chi)\right)$ if $\chi$ is generic.

Let $\rho: G_{K} \rightarrow \mathrm{GL}_{2}\left(\overline{\mathbf{F}}_{p}\right)$ be a reducible representation of the form $\rho \sim\left(\begin{array}{cc}\chi_{1} & * \\ 0 & \chi_{2}\end{array}\right)$ and $c_{\rho}$ an associated cohomology class. For $s \geqslant 1$ (respectively $s>1$ ), we say that $\rho$ has slope at most $s$ (respectively less than $s$ ) if $G_{K}^{u} \subset \operatorname{ker}(\rho)$ for all $u>s-1$ (respectively $u \geqslant s-1)$, or equivalently if $c_{\rho} \in \operatorname{Fil}^{s}\left(H^{1}\left(G_{K}, \overline{\mathbf{F}}_{p}(\chi)\right)\right.$ ) (respectively Fil $\left.^{<s} H^{1}\left(G_{K}, \overline{\mathbf{F}}_{p}(\chi)\right)\right)$. Note that $\rho$ is (at most) tamely ramified if $c_{\rho}$ is unramified; we say that $\rho$ is gently (respectively flatly, cogently, typically) ramified according to whether $c_{\rho}$ is. We remark that $[11,2.1]$ shows that if $\rho$ arises from a finite flat group scheme over $\mathcal{O}_{K}$, then $c_{\rho}$ is flatly ramified. If $\chi$ is cyclotomic, then our notion of flatly ramified coincides with Serre's notion of peu ramifiée in [24] recalled above.

\section{The Artin-Hasse exponential}

In this section we establish some properties of the Artin-Hasse exponential which strike us as having independent interest. Recall from, for example, [21, Section 7.2] that the Artin-Hasse exponential is defined by a power series with rational coefficients:

$$
E_{p}(x)=\exp \left(\sum_{n \geqslant 0} \frac{x^{p^{n}}}{p^{n}}\right) .
$$

Here, as usual, $\exp (x)=\sum_{n \geqslant 0}\left(x^{n} / n !\right)$. Since $p$ is fixed throughout, we omit the subscript and simply write is as $E(x)$. The denominators of the coefficients of $E(x)$ are prime to $p$, so we may regard $E(x) \in \mathbf{Z}_{p}[[x]]$, and hence as a function $E: B_{r}(0) \rightarrow B_{r}(1)$ for any $r<1$, where $B_{r}(a)$ denotes the open disk of radius $r$ of $a \in \mathbf{C}_{p}$. 
4.1. First multiplicativity lemma for $\boldsymbol{E}(\boldsymbol{x})$. Let $l$ be a finite field and let $L$ be the field of fractions of $W(l)$. For $a \in l$, let $[a] \in W(l)$ denote the Teichmüller lift of $a$. The following lemma establishes the key property of $E(x)$ we need; we use it to relate the additive structure of $l$ to the multiplicative structure of tamely ramified extensions of $L$.

LEMMA 4.1. If $a, b \in l$ then $E([a] x) E([b] x) E([a+b] x)^{-1} \in\left(W(l)[[x]]^{\times}\right)^{p}$.

Proof. For $n \geqslant 0$, we define elements $\delta_{n} \in L$ inductively as follows:

$$
\begin{aligned}
& \left.\delta_{0}=\frac{1}{p}([a]+[b]-[a+b])\right), \\
& \delta_{n}=\frac{1}{p^{n}}\left(\varphi^{n}\left(\delta_{0}\right)-\sum_{i=0}^{n-1} p^{i} \delta_{i}^{p^{n-i}}\right) \text { for } n \geqslant 1 .
\end{aligned}
$$

We claim that $\delta_{n} \in W(l)$ for all $n \geqslant 0$. The statement is clear for $n=0$, so suppose that $n>0$. For $i=0, \ldots, n-1$, we have $\delta_{i}^{p} \equiv \varphi\left(\delta_{i}\right) \bmod p$, and therefore

$$
\delta_{i}^{p^{n-i}}=\left(\delta_{i}^{p}\right)^{p^{n-1-i}} \equiv \varphi\left(\delta_{i}\right)^{p^{n-1-i}} \bmod p^{n-i} .
$$

By the definition of $\delta_{n-1}$ we have $\varphi^{n-1}\left(\delta_{0}\right)=\sum_{i=0}^{n-1} p^{i} \delta_{i}^{p^{n-1-i}}$, so

$$
\varphi^{n}\left(\delta_{0}\right)=\sum_{i=0}^{n-1} p^{i} \varphi\left(\delta_{i}\right)^{p^{n-1-i}} \equiv \sum_{i=0}^{n-1} p^{i} \delta_{i}^{p^{n-i}} \bmod p^{n},
$$

which gives the claim.

Now consider the power series

$$
f(x)=\prod_{i \geqslant 0} E\left(\delta_{i} x^{p^{i}}\right)
$$

which converges in $W(l)[[x]]$ since $E\left(x^{p^{i}}\right) \equiv 1 \bmod x^{p^{i}}$. We claim that

$$
E([a] x) E([b] x) E([a+b] x)^{-1}=f(x)^{p} .
$$

We prove this working in $L[[x]]$, where $\exp (g(x)) \exp (h(x))=\exp (g(x)+h(x))$ for $g(x), h(x) \in x L[[x]]$, and therefore $\exp \left(\sum_{i \geqslant 0} g_{i}(x)\right)=\prod_{i \geqslant 0} \exp \left(g_{i}(x)\right)$ if $g_{i}(x) \in x^{p^{i}} L[[x]]$. Note first that we have

$$
E([a] x) E([b] x) E([a+b] x)^{-1}=\exp \left(\sum_{n \geqslant 0} a_{n} x^{p^{n}}\right),
$$


where $a_{n}=p^{-n}\left([a]^{p^{n}}+[b]^{p^{n}}-[a+b]^{p^{n}}\right)=p^{1-n} \varphi^{n}\left(\delta_{0}\right)$. On the other hand

$$
\begin{aligned}
f(x)^{p} & =\prod_{i \geqslant 0} E\left(\delta_{i} x^{p^{i}}\right)^{p} \\
& =\prod_{i \geqslant 0} \exp \left(p \sum_{m \geqslant 0} p^{-m} \delta_{i}^{p^{m}} x^{p^{i+m}}\right) \\
& =\exp \left(\sum_{i, m \geqslant 0} p^{1-m} \delta_{i}^{p^{m}} x^{p^{i+m}}\right) \\
& =\exp \left(\sum_{n \geqslant 0} b_{n} x^{p^{n}}\right)
\end{aligned}
$$

where

$$
b_{n}=\sum_{i=0}^{n} p^{1+i-n} \delta_{i}^{p^{n-i}}=a_{n} .
$$

This proves the claim and hence the lemma.

4.2. Second multiplicativity lemma for $\boldsymbol{E}(\boldsymbol{x})$. We also need the following property of $E(x)$, which will be used to ensure that our constructions later are independent of various choices made.

LEMMA 4.2. If $\delta \in W(l)$, then

$$
E(x) E((1+p \delta) x)^{-1} \prod_{m \geqslant 0} E\left(p \delta x^{p^{m}}\right) \in\left(W(l)[[x]]^{\times}\right)^{p} .
$$

Proof. We have

$$
E((1+p \delta) x) E(x)^{-1}=\exp \left(\sum_{n \geqslant 0} a_{n} x^{p^{n}}\right)
$$

where

$$
a_{n}=\sum_{i=1}^{p^{n}}\left(\begin{array}{c}
p^{n} \\
i
\end{array}\right) p^{i-n} \delta^{i} .
$$

Note that $\left(\begin{array}{c}p^{n} \\ i\end{array}\right) p^{i-n}$ has valuation $i-v_{p}(i)$ for $i=1, \ldots, p^{n}$, so that $a_{n} \equiv p \delta \bmod$ $p^{2}$ if $p>2$, and $a_{n} \equiv 2\left(\delta+\delta^{2}\right) \bmod 4$ if $p=2$. On the other hand

$$
\prod_{m \geqslant 0} E\left(p \delta x^{p^{m}}\right)=\exp \left(\sum_{n \geqslant 0} b_{n} x^{p^{n}}\right)
$$


where

$$
b_{n}=\sum_{j=0}^{n} p^{p^{j}-j} \delta^{p^{j}}
$$

Setting $c_{n}=p^{-1}\left(b_{n}-a_{n}\right)$ gives $\exp \left(c_{n} x^{p^{n}}\right) \in W(l)[[x]]^{\times}$since $c_{n} \in p W(l)$, and

$$
E(x) E((1+p \delta) x)^{-1} \prod_{m \geqslant 0} E\left(p \delta x^{p^{m}}\right)=\left(\prod_{n \geqslant 0} \exp \left(c_{n} x^{p^{n}}\right)\right)^{p} .
$$

4.3. Homomorphisms induced by $\boldsymbol{E}(\boldsymbol{x})$. Suppose now that $M$ is a subfield of $\mathbf{C}_{p}$ containing $L$, and $\alpha \in M$ is such that $|\alpha|<1$. Note that Lemma 4.1 yields a homomorphism $\varepsilon: l \rightarrow \mathcal{O}_{L}[[x]]^{\times} \otimes \mathbf{F}_{p}$ defined by $\varepsilon([a])=E([a] x) \otimes 1$. We can therefore define a homomorphism

$$
\varepsilon_{\alpha}: l \otimes \overline{\mathbf{F}}_{p} \rightarrow \mathcal{O}_{M}^{\times} \otimes \overline{\mathbf{F}}_{p}
$$

as the extension of scalars of the composite of $\varepsilon$ with the multiplicative homomorphism $\mathcal{O}_{L}[[x]]^{\times} \rightarrow \mathcal{O}_{M}^{\times}$induced by evaluation at $\alpha$, so that $\varepsilon_{\alpha}(a \otimes b)=E([a] \alpha) \otimes b$.

In addition to properties of $\varepsilon_{\alpha}$ derived from Lemmas 4.1 and 4.2, we also need the following:

LEMMA 4.3. If $|\alpha|<p^{-1 / p(p-1)}$, then

$$
\varepsilon_{\alpha^{p}} \circ \text { Frob }=\varepsilon_{-p \alpha}
$$

where Frob is the automorphism of $l \otimes \overline{\mathbf{F}}_{p}$ induced by the absolute Frobenius on $l$.

Proof. It suffices to prove that if $\beta \in \mathcal{O}_{M}$ is such that $|\beta|<p^{-1 / p(p-1)}$, then $E\left(\beta^{p}\right) E(-p \beta)^{-1} \in\left(\mathcal{O}_{M}^{\times}\right)^{p}$. On the one hand we have $E\left(\beta^{p}\right)=\exp (-p \beta) E(\beta)^{p}$. On the other hand, setting $\gamma=\sum_{n \geqslant 1} p^{p^{n-n-1}}(-\beta)^{p^{n}}$, we see that $|\gamma|<p^{-1 /(p-1)}$, so $\exp (\gamma)$ converges to an element of $\mathcal{O}_{M}^{\times}$such that

$$
E(-p \beta)=\exp (-p \beta+p \gamma)=\exp (-p \beta) \exp (\gamma)^{p}
$$

\section{A basis for the cohomology}

We return to the setup of Section 3, so $K$ is an unramified extension of $\mathbf{Q}_{p}$ of degree $f$ with residue field $k, T$ is the set of embeddings $k \rightarrow \overline{\mathbf{F}}_{p}$, and $\chi$ is a character $G_{K} \rightarrow \overline{\mathbf{F}}_{p}^{\times}$. We use a homomorphism of the form (6) to construct an explicit basis for $H^{1}\left(G_{K}, \overline{\mathbf{F}}_{p}(\chi)\right)$. 
Let $M$ be a tamely ramified abelian extension of $K$ such that $\left.\chi\right|_{G_{M}}$ is trivial. We assume $M$ is of the form $L(\pi)$ where $L$ is an unramified extension of $K$ of degree prime to $p$ and $\pi$ is a uniformizer of $M$ such that $\pi^{e} \in K^{\times}$where the total ramification degree $e$ of $M$ divides $p^{f}-1$. We thus allow $M$ and $\pi$ to have a more general form than in the proof of Theorem 3.1, but note that we still have

$$
H^{1}\left(G_{K}, \overline{\mathbf{F}}_{p}(\chi)\right) \cong \operatorname{Hom}_{\operatorname{Gal}(M / K)}\left(M^{\times}, \overline{\mathbf{F}}_{p}(\chi)\right),
$$

which we identify with the $\overline{\mathbf{F}}_{p}$-dual of the vector space

$$
U_{\chi}=\left(M^{\times} \otimes \overline{\mathbf{F}}_{p}\left(\chi^{-1}\right)\right)^{\mathrm{Gal}(M / K)} .
$$

Our explicit basis for $H^{1}\left(G_{K}, \overline{\mathbf{F}}_{p}(\chi)\right)$ will be defined as the dual basis to one we construct for $U_{\chi}$.

5.1. Definition of $\boldsymbol{u}_{i}$. As in Section 3 we choose an embedding $\tau_{0}: k \rightarrow \overline{\mathbf{F}}_{p}$ and let $\tau_{i}=\tau_{0} \circ$ Frob $^{i}$, let $\left(a_{0}, \ldots, a_{f-1}\right)$ be the tame signature of $\chi$ and define the integers $n_{i}$ by (5), so that $\left.\chi\right|_{I_{K}}=\left.\omega_{f, i}^{n_{i}}\right|_{I_{K}}$. We define $\tau_{i}, a_{i}$ and $n_{i}$ for all $i \in \mathbf{Z}$ by requiring that they depend only on $i \bmod f$.

Since $\left.\chi\right|_{I_{K}}$ has order dividing $e$, we see that $n_{i}$ is divisible by $\left(p^{f}-1\right) / e$ for all $i$. Letting $\omega_{\pi}: \operatorname{Gal}(M / K) \rightarrow \mu_{e}(K) \subset K^{\times}$be the character defined by $\omega_{\pi}(g)=$ $g(\pi) / \pi$, we see that $\left.\bar{\omega}_{\pi}\right|_{I_{K}}=\left.\omega_{f}\right|_{I_{K}} ^{\left(p^{f}-1\right) / e}$, so that

$$
\left.\chi\right|_{I_{K}}=\left.\left(\tau_{i} \circ \bar{\omega}_{\pi}\right)\right|_{I_{K}} ^{e n_{i} /\left(p^{f}-1\right)} .
$$

We now define an embedding $\tau_{i}^{\prime}$ and an integer $n_{i}^{\prime}$ for each $i$. If $a_{i+1} \neq p$, then we set $\tau_{i}^{\prime}=\tau_{i+1}$ and $n_{i}^{\prime}=e n_{i+1} /\left(p^{f}-1\right)$. If $a_{i+1}=p$, then we let $j$ be the least integer greater than $i$ such that $a_{j+1} \neq p-1$; thus $\left(a_{i+1}, a_{i+2}, \ldots, a_{j}\right)=(p, p-1$, $\ldots, p-1)$, but $a_{j+1} \neq p-1$. We then set $\tau_{i}^{\prime}=\tau_{j+1}$ and $n_{i}^{\prime}=e n_{j+1} /\left(p^{f}-1\right)-e$. Note that for each $i$ we have $n_{i}^{\prime}>0$ and

$$
\chi=\mu\left(\tau_{i}^{\prime} \circ \bar{\omega}_{\pi}\right)^{n_{i}^{\prime}}
$$

for some unramified character $\mu: \operatorname{Gal}(L / K) \rightarrow \overline{\mathbf{F}}_{p}^{\times}$independent of $i$.

Recall that we have an isomorphism

$$
l \otimes \overline{\mathbf{F}}_{p} \cong \bigoplus_{\tau \in T}\left(l \otimes_{k, \tau} \overline{\mathbf{F}}_{p}\right)
$$

defined by the natural projection on to each component. By the Normal Basis Theorem, $l$ is free of rank one over $k[\mathrm{Gal}(L / K)]=k[\mathrm{Gal}(l / k)]$, so each 
component in the above decomposition is free of rank one over $\overline{\mathbf{F}}_{p}[\mathrm{Gal}(L / K)]$. It follows that for each embedding $\tau \in T$, the $\mu$-eigenspace

$$
\Lambda_{\tau, \mu}=\left\{a \in l \otimes_{k, \tau} \overline{\mathbf{F}}_{p} \mid g a=(1 \otimes \mu(g)) a \text { for all } g \in \operatorname{Gal}(L / K)\right\}
$$

is one-dimensional over $\overline{\mathbf{F}}_{p}$. Let $\lambda_{\tau, \mu}$ be a nonzero element of $\Lambda_{\tau, \mu}$. We now define

$$
u_{i}=\varepsilon_{\pi_{i}^{n^{\prime}}}\left(\lambda_{\tau_{i}^{\prime}, \mu}\right) \in \mathcal{O}_{M}^{\times} \otimes \overline{\mathbf{F}}_{p}
$$

for $i=0, \ldots, f-1$, where $\varepsilon_{\pi^{\prime}}$ is defined by (6).

For $g \in \operatorname{Gal}(M / K), a \in l, n \in \mathbf{Z}$, we have

$$
g\left(E\left([a] \pi^{n}\right)\right)=E\left(g\left([a] \pi^{n}\right)\right)=E\left(\omega_{\pi}(g)^{n} g([a]) \pi^{n}\right),
$$

so that

$$
g\left(\varepsilon_{\pi^{n}}(\lambda)\right)=\varepsilon_{\pi^{n}}\left(\left(\bar{\omega}_{\pi}(g)^{n} \otimes 1\right) g(\lambda)\right)
$$

for all $\lambda \in l \otimes \overline{\mathbf{F}}_{p}$. Since

$$
\left(\bar{\omega}_{\pi}(g)^{n_{i}^{\prime}} \otimes 1\right) g\left(\lambda_{\tau_{i}^{\prime}, \mu}\right)=\left(\bar{\omega}_{\pi}(g)^{n_{i}^{\prime}} \otimes \mu(g)\right) \lambda_{\tau_{i}^{\prime}, \mu}=(1 \otimes \chi(g)) \lambda_{\tau_{i}^{\prime}, \mu},
$$

we conclude that $g u_{i}=(1 \otimes \chi(g)) u_{i}$ for all $g \in \operatorname{Gal}(M / K)$. We can therefore view $u_{i}$ as an element of $U_{\chi}$.

5.2. Definition of $\boldsymbol{u}_{\text {triv }}$ and $\boldsymbol{u}_{\text {cyc }}$. We now define additional classes in $U_{\chi}$ in the case that $\chi$ is trivial or cyclotomic. Note that if $g \in \operatorname{Gal}(M / K)$, then $g(\pi)=\omega_{\pi}(g) \pi$ and $\omega_{\pi}(g) \in \mu_{e}(K)$, which is contained in $\left(M^{\times}\right)^{p}$. It follows that $g(\pi \otimes 1)=\pi \otimes 1$ in $M^{\times} \otimes \overline{\mathbf{F}}_{p}$, so that

$$
u_{\text {triv }}=\pi \otimes 1
$$

defines an element of $U_{\chi}$ for the trivial character $\chi$.

If $\chi$ is cyclotomic then the assumption that $\left.\chi\right|_{G_{M}}$ is trivial ensures that $\mathbf{Q}_{p}\left(\zeta_{p}\right) \subset M$; in particular $e$ is divisible by $p-1$. We now determine which elements $\alpha \in 1+\pi^{e p /(p-1)} \mathcal{O}_{M}$ are $p$ th-powers. Recall that $\mathbf{Q}_{p}\left(\zeta_{p}\right)=\mathbf{Q}_{p}(\delta)$ where $\delta^{p-1}=-p$, so we may write $\alpha$ in the form $1+\beta \delta^{p}$ with $\beta \in \mathcal{O}_{M}$. We claim that $\alpha=1+\beta \delta^{p} \in\left(M^{\times}\right)^{p}$ if and only if $\operatorname{tr}_{l / \mathbf{F}_{p}} \bar{\beta}=0$. Suppose first that $\operatorname{tr}_{l / \mathbf{F}_{p}} \bar{\beta}=0$. We can then write $\bar{\beta}=\bar{\gamma}-\bar{\gamma}^{p}$ for some $\bar{\gamma} \in l$. (This follows for example from the fact that $H^{1}\left(\operatorname{Gal}\left(l / \mathbf{F}_{p}\right), l\right)=0$ since $l$ is free over $\mathbf{F}_{p}\left[\mathrm{Gal}\left(l / \mathbf{F}_{p}\right)\right]$ by the Normal Basis Theorem. Alternatively, note that $\operatorname{tr}_{l / \mathbf{F}_{p}}$ is surjective since $l$ is separable over $\mathbf{F}_{p}$, so counting dimensions shows that $l \rightarrow l \rightarrow \mathbf{F}_{p}$ is exact, where the maps 
are $1-$ Frob and $\operatorname{tr}_{l / \mathbf{F}_{p}}$.) We can therefore write $\beta \equiv \gamma-\gamma^{p} \bmod \pi \mathcal{O}_{M}$ for some $\gamma \in \mathcal{O}_{M}$, so that

$$
\alpha(1+\gamma \delta)^{p} \equiv 1+\left(\beta-\gamma+\gamma^{p}\right) \delta^{p} \equiv 1 \bmod \pi^{1+e p /(p-1)} \mathcal{O}_{M} .
$$

Since $\exp \left(p^{-1} \log x\right)$ converges to a $p^{\text {th }}$ root of $x$ if $|x-1|<p^{-p /(p-1)}$, it follows that $\alpha(1+\gamma \delta)^{p} \in\left(\mathcal{O}_{M}^{\times}\right)^{p}$, and hence that $\alpha \in\left(\mathcal{O}_{M}^{\times}\right)^{p}$. Suppose conversely that $\alpha=1+\beta \delta^{p} \in\left(M^{\times}\right)^{p}$. Then considering valuations as in the proof of Theorem 3.1, we see that $\alpha=(1+\gamma \delta)^{p}$ for some $\gamma \in \mathcal{O}_{M}$. Since

$$
(1+\gamma \delta)^{p} \equiv 1+\left(\gamma^{p}-\gamma\right) \delta^{p} \bmod \delta^{p+1} \mathcal{O}_{M},
$$

we deduce that $\beta \equiv \gamma^{p}-\gamma \bmod \pi$, and hence that $\operatorname{tr}_{l / \mathbf{F}_{p}}(\bar{\beta})=0$. This completes the proof of the claim. Now choose any $b \in l$ such that $\operatorname{tr}_{l / \mathbf{F}_{p}} b \neq 0$, and define

$$
u_{\mathrm{cyc}}=\varepsilon_{\delta^{p}}(b \otimes 1)=E\left([b] \delta^{p}\right) \otimes 1,
$$

which is a nontrivial element of $\mathcal{O}_{M}^{\times} \otimes \overline{\mathbf{F}}_{p}$ by the above claim. Moreover, since $\operatorname{tr}_{l / \mathbf{F}_{p}}(g b)=\operatorname{tr}_{l / \mathbf{F}_{p}}(b)$ for all $g \in \operatorname{Gal}(M / K)$, it also follows from the claim and Lemma 4.1 that $E\left(g([b]) \delta^{p}\right) E\left([b] \delta^{p}\right)^{-1} \in\left(\mathcal{O}_{M}^{\times}\right)^{p}$, so $\varepsilon_{\delta^{p}}(g b \otimes 1)=\varepsilon_{\delta^{p}}(b \otimes 1)$. Since $g(\delta)=[\chi(g)] \delta$, we see as in the construction of the elements $u_{i}$ that

$$
g\left(u_{\mathrm{cyc}}\right)=\varepsilon_{\delta^{p}}(\chi(g) g b \otimes 1)=(1 \otimes \chi(g)) \varepsilon_{\delta^{p}}(g b \otimes 1)=(1 \otimes \chi(g)) u_{\mathrm{cyc}},
$$

and therefore that $u_{\mathrm{cyc}} \in U_{\chi}$.

\subsection{Bases for $U_{\chi}$ and $H^{1}\left(G_{K}, \overline{\mathbf{F}}_{p}(\chi)\right)$.}

THEOREM 5.1. Let $B$ denote the subset of $U_{\chi}$ consisting of the elements $u_{i}$ for $i=0, \ldots, f-1$, together with $u_{\text {triv }}$ if $\chi$ is trivial and $u_{\text {cyc }}$ if $\chi$ is cyclotomic. Then $B$ is a basis for $U_{\chi}$.

Before giving the proof, we remark that if $p=2$, then the cyclotomic character is trivial, so the basis $B$ includes both $u_{\text {triv }}$ and $u_{\text {cyc }}$ and hence consists of $f+2$ elements.

Proof. Define a decreasing filtration on $U_{\chi}$ with $\mathrm{Fil}^{0} U_{\chi}=U_{\chi}$ and $\mathrm{Fil}^{m} U_{\chi}$ as the image of $\left(U_{m} \otimes \overline{\mathbf{F}}_{p}\left(\chi^{-1}\right)\right)^{\mathrm{Gal}(M / K)}$ for $m \geqslant 1$, where $U_{m}=1+\pi^{m} \mathcal{O}_{M}$. Setting $\mathrm{gr}^{m} U_{\chi}=\mathrm{Fil}^{m} U_{\chi} / \mathrm{Fil}^{m+1} U_{\chi}$, we see as in the proof of Theorem 3.1 that

$$
\operatorname{dim}_{\overline{\mathbf{F}}_{p}} \operatorname{gr}^{m} U_{\chi}=\operatorname{dim}_{\overline{\mathbf{F}}_{p}} \operatorname{gr}^{s} H^{1}\left(G_{K}, \overline{\mathbf{F}}_{p}(\chi)\right)
$$


where $s=1+m / e$ if $m \geqslant 1$, and $s=0$ if $m=0$. Recall that these dimensions $d_{s}$ are given by Theorem 3.1. We prove that for each $m \geqslant 0$, there are $d_{s}$ elements of $B \cap \mathrm{Fil}^{m} U_{\chi}$ whose images in $\mathrm{gr}^{m} U_{\chi}$ are linearly independent. It then follows that $B$ spans $U_{\chi}$, which suffices since the cardinality of $B$ coincides with the dimension of $U_{x}$.

If $m>p e /(p-1)$, then $s>1+p /(p-1)$, so $d_{s}=0$ and there is nothing to prove.

If $m=p e /(p-1)$, then $s=1+p /(p-1)$, so $d_{s}=0$ unless $\chi$ is cyclotomic in which case $d_{s}=1$. Therefore, it suffices to note that $u_{\mathrm{cyc}}$ is a nontrivial element of $\mathrm{Fil}^{p e /(p-1)} U_{\chi}$.

Now suppose $e /(p-1) \leqslant m<e p /(p-1)$, so $d_{s}$ is the number of $i$ such that $s=1+m / e=1+n_{i+1} /\left(p^{f}-1\right)$ and $a_{i+1} \neq p$. For each such $i$, we have $n_{i}^{\prime}=e n_{i+1} /\left(p^{f}-1\right)=m$ and $\tau_{i}^{\prime}=\tau_{i+1}$, so that $u_{i}=\varepsilon_{\pi^{m}}\left(\lambda_{\tau_{i+1}, \mu}\right) \in \mathrm{Fil}^{m} U_{\chi}$. We now show that the images of these $u_{i}$ in $\operatorname{gr}^{m} U_{\chi}$ are linearly independent. We may assume that $d_{s}>0$ and hence that $m$ is not divisible by $p$. Since $m<p e /(p-1)$, we see as in the proof of Theorem 3.1 that the natural map

$$
U_{m} / U_{m+1} \rightarrow\left(M^{\times} / U_{m+1}\right) \otimes \mathbf{F}_{p}
$$

is injective, so that we may identify $\operatorname{gr}^{m} U_{\chi}$ with

$$
\left(U_{m} / U_{m+1} \otimes \overline{\mathbf{F}}_{p}\left(\chi^{-1}\right)\right)^{\mathrm{Gal}(M / K)} .
$$

Since the map

$$
l \otimes \overline{\mathbf{F}}_{p} \rightarrow U_{m} / U_{m+1} \otimes \overline{\mathbf{F}}_{p}
$$

induced by $\varepsilon_{\pi^{m}}$ is an isomorphism and the elements $\lambda_{\tau_{i+1}, \mu}$ are linearly independent over $\overline{\mathbf{F}}_{p}$, it follows that so are their images in $\mathrm{gr}^{m} U_{\chi}$.

Now suppose that $0<m<e /(p-1)$. In this case $d_{s}$ is the number of $i$ such that $s=1+m / e=n_{j+1} /\left(p^{f}-1\right), a_{j+1} \neq p-1$, and $\left(a_{i+1}, \ldots, a_{j}\right)=(p, p-1$, $\ldots, p-1)$ for some $j>i$. For each such $i$, we have $n_{i}^{\prime}=e n_{j+1} /\left(p^{f}-1\right)-e=m$ and $\tau_{i}^{\prime}=\tau_{j+1}$, so that $u_{i}=\varepsilon_{\pi^{m}}\left(\lambda_{\tau_{j+1}, \mu}\right) \in \mathrm{Fil}^{m} U_{\chi}$. Note also that for distinct $i$, the corresponding $j$ are distinct mod $f$. The proof that the images of $u_{i}$ in $\operatorname{gr}^{m} U_{\chi}$ are linearly independent is then the same as in the preceding case.

Finally note that if $m=0$, then $s=0$, so $d_{s}=0$ unless $\chi$ is trivial, in which case $d_{s}=1$. Therefore, it suffices to note that $u_{\text {triv }}$ is not in Fil $^{1} U_{\chi}$.

We can now define a basis for $H^{1}\left(G_{K}, \overline{\mathbf{F}}_{p}(\chi)\right)$ as the dual basis to the one in Theorem 5.1, denoting the corresponding cohomology classes $c_{\tau}$ for $\tau: k \rightarrow \overline{\mathbf{F}}_{p}$, together with $c_{\text {un }}$ if $\chi$ is trivial and $c_{\text {tr }}$ if $\chi$ is cyclotomic. We record the construction as follows: 
COROLLARY 5.2. The set consisting of the classes $c_{\tau}$ for $\tau \in T$, together with $c_{\mathrm{un}}$ if $\chi$ is trivial and $c_{\mathrm{tr}}$ if $\chi$ is cyclotomic, forms a basis for $H^{1}\left(G_{K}, \overline{\mathbf{F}}_{p}(\chi)\right)$.

\section{Dependent pairs and admissible subsets}

We now determine the extent to which the basis for $H^{1}\left(G_{K}, \overline{\mathbf{F}}_{p}(\chi)\right)$ just constructed is independent (up to scalars) of the choices made. We maintain the notation of Section 5, so $K$ is an unramified extension of $\mathbf{Q}_{p}$ of degree $f$ with residue field $k, T=\left\{\tau_{0}, \ldots, \tau_{f-1}\right\}$ (where $\tau_{i}=\tau_{0} \circ$ Frob $^{i}$ ) is the set of embeddings $k \rightarrow \overline{\mathbf{F}}_{p}$, and we fix a character $\chi: G_{K} \rightarrow \overline{\mathbf{F}}_{p}^{\times}$and write $\left.\chi\right|_{I_{K}}=\prod_{i=0}^{f} \omega_{\tau_{i}}^{a_{i}}$ where $\omega_{\tau_{i}}: I_{K} \rightarrow \overline{\mathbf{F}}_{p}^{\times}$corresponds to $\tau_{i}$ by local class field theory and $\left(a_{0}, \ldots, a_{f-1}\right)$ is the tame signature of $\chi$.

6.1. Dependent pairs. Recall that we chose an extension $M$ of $K$ with a uniformizer $\pi$ such that $\left.\chi\right|_{G_{M}}$ is trivial and $M=L(\pi)$ where $L / K$ is unramified of degree prime to $p, e=[M: L]$ divides $p^{f}-1$, and $\pi^{e} \in K^{\times}$. We introduce the following notion in order to explain how the basis of Corollary 5.2 depends on the choice of $M$ and $\pi$ :

Definition 6.1. For $i, t \in \mathbf{Z}$ with $1 \leqslant t \leqslant f-1$, we say that $([i],[i+t]) \in$ $(\mathbf{Z} / f \mathbf{Z})^{2}$ is a dependent pair if $a_{i+1}=p, a_{i+t+1} \neq p$, and

$$
a_{i+2}=\cdots=a_{i+s}=p-1, \quad a_{i+s+1}=\cdots=a_{i+t}=p
$$

for some $s \in 1, \ldots, t$.

Note that the first (respectively second) displayed chain of equations automatically holds if $s=1$ (respectively $s=t$ ). Note that if $a_{i+1} \neq p$ then there are no dependent pairs of the form ([i], $[j])$, and that if $a_{i+1}=p$ then the number of dependent pairs $([i],[j])$ is either $s$ or $s-1$ where $s \in\{1, \ldots, f\}$ is such that

$$
a_{i+2}=\cdots=a_{i+s}=p-1, \quad a_{i+s+1} \neq p-1 .
$$

More precisely, the number of such dependent pairs is $s$ unless

$$
\left(a_{i+2}, \ldots, a_{i+s}, a_{i+s+1}, \ldots, a_{i+f+1}\right)=(p-1, \ldots, p-1, p, \ldots, p),
$$

in which case it is $s-1$. Note that there are no dependent pairs at all if $\left.\chi\right|_{I_{K}}$ is trivial (in which case all $a_{i}=p-1$ ) or cyclotomic (in which case all $a_{i}=1$ ). 
6.2. Dependence of the basis on the choice of uniformizer. Recall that we defined a basis for $U_{\chi}=\left(M^{\times} \otimes \overline{\mathbf{F}}_{p}\left(\chi^{-1}\right)\right)^{\mathrm{Gal}(M / K)}$ using the elements $u_{i}$ (for $i=0, \ldots, f-1), u_{\text {triv }}$ (if $\chi$ is trivial) and $u_{\text {cyc }}$ (if $\chi$ is cyclotomic) defined by Equations (7)-(9). Suppose now that we choose another uniformizer $\pi^{\prime}$ (for the same $M$ ) such that $\left(\pi^{\prime}\right)^{e} \in K^{\times}$and accordingly define elements $u_{i}^{\prime}$ for $i=0, \ldots$, $f-1$, and $u_{\text {triv }}^{\prime}$ if $\chi$ is trivial. (Note that $u_{\text {cyc }}$ does not depend on the choice of uniformizer.)

Proposition 6.2. For $i=0, \ldots, f-1$, the element $u_{i}^{\prime}$ differs from a nonzero multiple of $u_{i}$ by an element of the span of

$$
\left\{u_{j} \mid([i],[j]) \text { is a dependent pair }\right\}
$$

and $u_{\mathrm{cyc}}$ if $\chi$ is cyclotomic.

Proof. Recall that we require $\pi^{e}$ and $\left(\pi^{\prime}\right)^{e}$ to be in $K$, so setting $\alpha=\pi^{\prime} / \pi$ and $a=\bar{\alpha} \in l$, we have $\alpha^{e} \in \mathcal{O}_{K}^{\times}$and $a^{e} \in k^{\times}$.

Suppose first that $\alpha=[a]$. Note that $\bar{\omega}_{\pi^{\prime}}=\bar{\omega}_{\pi} \omega_{a}$, where $\omega_{a}$ is the unramified character of $\operatorname{Gal}(L / K) \cong \operatorname{Gal}(l / k)$ sending $g$ to $g(a) / a \in \mu_{e}(k)$. Writing

$$
\chi=\mu\left(\tau_{i}^{\prime} \circ \bar{\omega}_{\pi}\right)^{n_{i}^{\prime}}=\mu^{\prime}\left(\tau_{i}^{\prime} \circ \bar{\omega}_{\pi^{\prime}}\right)^{n_{i}^{\prime}},
$$

we see $\mu=\mu^{\prime}\left(\tau_{i}^{\prime} \circ \omega_{a}\right)^{n_{i}^{\prime}}$. Recall that $u_{i}$ and $u_{i}^{\prime}$ are defined by

$$
u_{i}=\varepsilon_{\pi_{i}^{n_{i}^{\prime}}}\left(\lambda_{\tau_{i}^{\prime}, \mu}\right) \quad \text { and } \quad u_{i}^{\prime}=\varepsilon_{\left(\pi^{\prime}\right)^{n_{i}^{\prime}}}\left(\lambda_{\tau_{i}^{\prime}, \mu^{\prime}}\right),
$$

where $\lambda_{\tau_{i}^{\prime}, \mu}$ and $\lambda_{\tau_{i}^{\prime}, \mu^{\prime}}$ are any nonzero vectors in the corresponding eigenspaces $\Lambda_{\tau_{i}^{\prime}, \mu}$ and $\Lambda_{\tau_{i}^{\prime}, \mu^{\prime}}$. Note however that $\Lambda_{\tau_{i}^{\prime}, \mu}=\left(a^{n_{i}^{\prime}} \otimes 1\right) \Lambda_{\tau_{i}^{\prime}, \mu^{\prime}}$, so we may choose $\lambda_{\tau_{i}^{\prime}, \mu}=\left(a^{n_{i}^{\prime}} \otimes 1\right) \lambda_{\tau_{i}^{\prime}, \mu^{\prime}}$, which gives $u_{i}=u_{i}^{\prime}$.

The preceding paragraph shows that we may replace $\pi$ by $[a] \pi$ and hence assume that $\alpha \equiv 1 \bmod \pi \mathcal{O}_{M}$. Note that $\bar{\omega}_{\pi^{\prime}}=\bar{\omega}_{\pi}$, so $\mu=\mu^{\prime}$ and we may use the same $\lambda_{\tau_{i}^{\prime}, \mu}$ in the definitions of $u_{i}$ and $u_{i}^{\prime}$. Since $\alpha^{e} \in 1+p \mathcal{O}_{K}$, we see that in fact $\alpha \in 1+p \mathcal{O}_{K}$, so that $\left(\pi^{\prime}\right)^{n_{i}^{\prime}}=\pi^{n_{i}^{\prime}} \alpha^{n_{i}^{\prime}}$ where $\alpha^{n_{i}^{\prime}}=1+p \delta$ for some $\delta \in \mathcal{O}_{K}$.

We now apply Lemma 4.2 with $x$ evaluated at $\pi^{n_{i}^{\prime}}$. First note that if $a_{i+1} \neq p$, then $n_{i}^{\prime} \geqslant e /(p-1)$, so $E\left([a] p \delta\left(\pi^{n_{i}^{\prime}}\right)^{p^{m}}\right) \equiv 1 \bmod \pi^{e p /(p-1)}$ for all $a \in l$ and $m \geqslant 0$. The lemma then implies that $u_{i}^{\prime}-u_{i} \in \mathrm{Fil}^{e p /(p-1)} U_{\chi}$, so $u_{i}=u_{i}^{\prime}$ unless $\chi$ is cyclotomic, in which case $u_{i}^{\prime}-u_{i}$ is in the span of $u_{\text {cyc }}$.

Now suppose that $a_{i+1}=p$ and let $s$ be the least positive integer such that $a_{i+s+1} \neq p-1$. We then have $n_{i}^{\prime}=e n_{i+s+1} /\left(p^{f}-1\right)-e$, where

$$
n_{i+s+1}=a_{i+s+1}+a_{i+s+2} p+\cdots+a_{i} p^{f-s-1}+p^{f} .
$$


For $m=0, \ldots, s-1$, we see that

$$
p^{m}\left(n_{i+s+1}-\left(p^{f}-1\right)\right)=n_{i+s-m+1}-\left(p^{f}-1\right) .
$$

Since $n_{i+2} \geqslant 1+p+\cdots p^{f-2}+p^{f}$, we see also that $p^{s}\left(n_{i+s+1}-\left(p^{f}-1\right)\right)=$ $p\left(n_{i+2}-\left(p^{f}-1\right)\right)>\left(p^{f}-1\right) /(p-1)$, and hence that $p^{s} n_{i}^{\prime}>e /(p-1)$. Therefore, $E\left([a] p \delta\left(\pi^{n_{i}^{\prime}}\right)^{p^{m}}\right)$ is in $\left(\mathcal{O}_{M}^{\times}\right)^{p}$ for all $a \in l$ and $m \geqslant s$, so Lemma 4.2 implies that

$$
\varepsilon_{\left(\pi^{\prime}\right)^{n_{i}^{\prime}}}=\varepsilon_{\pi^{n_{i}^{\prime}}}+\sum_{m=0}^{s-1} \varepsilon_{p \delta \pi^{n_{i}^{\prime}} p^{m}} \circ \text { Frob }^{m} .
$$

Note also that $p \delta \pi^{n_{i}^{\prime}} p^{m}=\beta \pi^{n_{i}^{\prime}} p^{m}+e$ for some $\beta \in \mathcal{O}_{K}$, and another application of

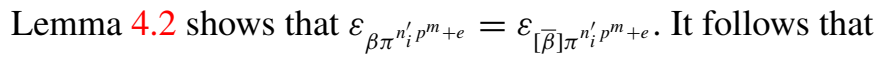

$$
u_{i}^{\prime}-u_{i}=\tau_{i+s+1}(\bar{\beta}) \sum_{m=0}^{s-1} \varepsilon_{\pi_{i}^{n_{i}^{\prime} m^{m}+e}}\left(\operatorname{Frob}^{m}\left(\lambda_{\tau_{i+s+1}, \mu}\right)\right) .
$$

We show that each term is a multiple of a vector of the form $u_{i+t}$, where either $t \in\{1, \ldots, s-1\}$, or $t$ is the least integer such that $t \geqslant s$ and $a_{i+t+1} \neq p$. Note that $([i],[i+t])$ is a dependent pair for each such $t$ (including $t=f+1$ in the case $\left.\left(a_{i+2}, \ldots, a_{i+s}, a_{i+s+1}, \ldots, a_{i+f+1}\right)=(p-1, \ldots, p-1, p, \ldots, p)\right)$.

First consider the term with $m=0$, and note that $n_{i}^{\prime}+e=e n_{i+s+1} /\left(p^{f}-1\right)$. If $a_{i+s+1} \neq p$, then $n_{i+s}^{\prime}=e n_{i+s+1} /\left(p^{f}-1\right)$ and $\tau_{i+s}^{\prime}=\tau_{i+s+1}$, so $\varepsilon_{\pi_{i}^{n_{i}^{\prime}+e}}\left(\lambda_{\tau_{i+s+1}, \mu}\right)$ $=u_{i+s}$. On the other hand, if $a_{i+s+1}=p$, then $n_{i+s+1}$ is divisible by $p$ and $e n_{i+s+1} / p\left(p^{f}-1\right)>e / p(p-1)$, so Lemma 4.3 implies that

$$
\varepsilon_{\pi^{e n_{i+s+1} /\left(p^{f}-1\right)}}=\varepsilon_{-p \pi^{e n_{i+s+1} / p\left(p^{f}-1\right)}} \circ \text { Frob }^{-1} .
$$

Writing $-p=\gamma \pi^{e}$ for some $\gamma \in \mathcal{O}_{K}^{\times}$and noting that $n_{i+s+2}=n_{i+s+1} / p+p^{f}-1$, we see that this is the same as $\varepsilon_{\gamma \pi^{e n_{i+s+2} /\left(p^{f}-1\right)}} \circ \mathrm{Frob}^{-1}$, and another application of Lemma 4.2 shows that we may replace $\gamma$ by $[\bar{\gamma}]$. Since Frob ${ }^{-1}$ sends $\Lambda_{\tau_{i+s+1}, \mu}$ to $\Lambda_{\tau_{i+s+2}, \mu}$, we conclude that $\varepsilon_{\pi^{n_{i}^{\prime}+e}}\left(\lambda_{\tau_{i+s+1}, \mu}\right)$ is a scalar multiple of $\varepsilon_{\pi^{e n_{i+s+2} /\left(p^{f}-1\right)}}\left(\lambda_{\tau_{i+s+2}, \mu}\right)$. If $a_{i+s+2} \neq p$, then this is $u_{i+s+1}$. If $a_{i+s+2}=p$, then we may iterate the argument to conclude that $\varepsilon_{\pi_{i}^{n_{i}^{\prime}+e}}\left(\lambda_{\tau_{i+s+1}, \mu}\right)$ is a multiple of $u_{i+t}$, where $t$ is the least integer such that $t \geqslant s$ and $a_{i+t+1} \neq p$.

Finally for $m=1, \ldots, s-1$, we have $a_{i+s-m+1}=p-1 \neq p$, so

$$
n_{i+s-m}^{\prime}=\frac{e n_{i+s-m+1}}{p^{f}-1}=\frac{e}{p^{f}-1}\left(p^{m}\left(n_{i+s+1}-\left(p^{f}-1\right)\right)+p^{f}-1\right)=n_{i}^{\prime} p^{m}+e
$$

and $\tau_{i+s-m}^{\prime}=\tau_{i+s-m+1}$. Since Frob ${ }^{m}$ sends $\Lambda_{\tau_{i+s+1}, \mu}$ to $\Lambda_{\tau_{i+s-m+1}, \mu}$, we conclude that $\varepsilon_{\pi_{i}^{n^{\prime} p^{m}+e}}\left(\operatorname{Frob}^{m}\left(\lambda_{\tau_{i+s+1}, \mu}\right)\right)$ is a multiple of $u_{i+t}$ where $t=s-m$. 
6.3. Dependence of the dual basis on the choice of $\boldsymbol{M}$. Recall that we defined a basis for $H^{1}\left(G_{K}, \overline{\mathbf{F}}_{p}(\chi)\right)$ as the dual basis to the one constructed for $U_{\chi}$, denoting the corresponding cohomology classes $c_{\tau}$ for $\tau: k \rightarrow \overline{\mathbf{F}}_{p}$, together with $c_{\text {un }}$ if $\chi$ is trivial and $c_{\text {tr }}$ if $\chi$ is cyclotomic. Suppose now that we make another choice of $M^{\prime}$ and $\pi^{\prime}$ of the required form and denote the corresponding basis elements $c_{\tau}^{\prime}$ for $\tau: k \rightarrow \overline{\mathbf{F}}_{p}$, and $c_{\mathrm{tr}}^{\prime}$ if $\chi$ is cyclotomic. (Note that if $\chi$ is trivial, then $c_{\text {un }}$ spans $H_{\text {un }}^{1}\left(G_{K}, \overline{\mathbf{F}}_{p}\right)$, so, up to scalar, is independent of the choices.)

Proposition 6.3. For $i=0, \ldots, f-1$, the element $c_{\tau_{i}}^{\prime}$ differs from a nonzero multiple of $c_{\tau_{i}}$ by an element of the span of

$$
\left\{c_{\tau_{j}} \mid([j],[i]) \text { is a dependent pair }\right\}
$$

and $c_{\mathrm{un}}$ if $\chi$ is trivial.

Proof. Suppose first that the $c_{\tau}$ and $c_{\tau}^{\prime}$ are defined using the same field $M$, but different choices of uniformizers $\pi$ and $\pi^{\prime}$. Suppose also that $\chi$ is not trivial or cyclotomic. Define $T=\left(t_{i j}\right) \in \mathrm{GL}_{f}\left(\overline{\mathbf{F}}_{p}\right)$ by $u_{i}=\sum_{i=0}^{f-1} t_{i j} u_{j}^{\prime}$ for $i=0, \ldots, f-1$, so that $c_{\tau_{i}}^{\prime}=\sum_{j=0}^{f-1} t_{j i} c_{\tau_{j}}^{\prime}$ for $i=0, \ldots, f-1$. The conclusion is then immediate from Proposition 6.2, which shows that $t_{i i} \neq 0$ for each $i$, and that $t_{i j}=0$ unless $i=j$ or $([i],[j])$ is a dependent pair. If $\chi$ is trivial or cyclotomic, then there are no dependent pairs, and the conclusion is again immediate from Proposition 6.2.

Now suppose that $M$ and $M^{\prime}$ are any two extensions of $K$ of the required form. By symmetry, we may replace $M^{\prime}$ with a larger extension satisfying the hypotheses, and hence assume that if $M=L(\pi)$, then $M^{\prime}=L^{\prime}\left(\pi^{\prime}\right)$ where $L^{\prime}$ is an unramified extension of $L$ of degree prime to $p$ and $\left(\pi^{\prime}\right)^{d}=\pi$ where $d e$ divides $p^{f}-1$. By the preceding paragraph, we may assume that the $c_{\tau}^{\prime}$ are defined using the uniformizer $\pi^{\prime}$.

Note that we have used the isomorphisms of class field theory in order to identify $H^{1}\left(G_{K}, \overline{\mathbf{F}}_{p}(\chi)\right)$ with both $\operatorname{Hom}_{\overline{\mathbf{F}}_{p}}\left(U_{\chi}, \overline{\mathbf{F}}_{p}\right)$ and with $\operatorname{Hom}_{\overline{\mathbf{F}}_{p}}\left(U_{\chi}^{\prime}, \overline{\mathbf{F}}_{p}\right)$, where

$$
U_{\chi}=\left(M^{\times} \otimes \overline{\mathbf{F}}_{p}\left(\chi^{-1}\right)\right)^{\mathrm{Gal}(M / K)} \quad \text { and } \quad U_{\chi}^{\prime}=\left(\left(M^{\prime}\right)^{\times} \otimes \overline{\mathbf{F}}_{p}\left(\chi^{-1}\right)\right)^{\mathrm{Gal}\left(M^{\prime} / K\right)} .
$$

Recall that this identification is compatible with the isomorphism $U_{\chi}^{\prime} \rightarrow U_{\chi}$ induced by the norm map from $\left(M^{\prime}\right)^{\times}$to $M^{\times}$. Denoting this isomorphism $v_{M^{\prime} / M}$ and the basis elements for $U_{\chi}^{\prime}$ by $u_{i}^{\prime}$, it suffices to prove that $v_{M^{\prime} / M}\left(u_{i}^{\prime}\right)$ is a multiple of $u_{i}$ for $i=0, \ldots, f-1$, and similarly for $u_{\mathrm{cyc}}^{\prime}$ and $u_{\mathrm{cyc}}$ if $\chi$ is cyclotomic.

With our choices of $\pi$ and $\pi^{\prime}$, the map $\varepsilon_{\pi^{n_{i}^{\prime}}}$ appearing in the definition of $u_{i}$ is simply the restriction to $l \otimes \overline{\mathbf{F}}_{p}$ of the one in the definition of $u_{i}^{\prime}$. Note also that the embeddings $\tau_{i}^{\prime}$ and unramified characters $\mu$ are the same for $M$ and $M^{\prime}$. 
Therefore,

$$
v_{M^{\prime} / M}\left(u_{i}^{\prime}\right)=\sum_{g \in \operatorname{Gal}\left(M^{\prime} / M\right)} \varepsilon_{\pi_{i}^{n_{i}^{\prime}}} \lambda_{\tau_{i}^{\prime}, \mu}^{\prime}=d \varepsilon_{\pi^{n_{i}}} \operatorname{tr}_{l^{\prime} / l}\left(\lambda_{\tau_{i}^{\prime}, \mu}^{\prime}\right)
$$

where $\lambda_{\tau_{i}^{\prime}, \mu}^{\prime}$ is in the $\mu$-eigenspace for the action of $\operatorname{Gal}\left(l^{\prime} / k\right)$ on $l^{\prime} \otimes_{k, \tau_{i}^{\prime}} \overline{\mathbf{F}}_{p}$. The conclusion follows from the observation that $\operatorname{tr}_{l^{\prime} / l}\left(\lambda_{\tau_{i}^{\prime}, \mu}^{\prime}\right) \in \Lambda_{\tau_{i}^{\prime}, \mu}$. Finally, if $\chi$ is cyclotomic, then the argument for $u_{\text {cyc }}^{\prime}$ is similar.

\subsection{Admissible sets.}

Definition 6.4. We say that a subset $J \subset \mathbf{Z} / f \mathbf{Z}$ is admissible if for all dependent pairs $([j],[i])$, we have that if $[i] \in J$, then $[j] \in J$. We say that a subset $J \subset T$ is admissible if the corresponding subset of $\mathbf{Z} / f \mathbf{Z}$ is admissible.

The following is immediate from Proposition 6.3:

COROllary 6.5. If $J \subset T$ is admissible, then the span of the set $\left\{c_{\tau} \mid \tau \in J\right\}$ in $H^{1}\left(G_{K}, \overline{\mathbf{F}}_{p}(\chi)\right) / H_{\mathrm{ur}}^{1}\left(G_{K}, \overline{\mathbf{F}}_{p}(\chi)\right)$ is well defined, that is, independent of the choice of $M$ and $\pi$.

Finally we give some criteria for admissibility in terms of the subspaces of $H^{1}\left(G_{K}, \overline{\mathbf{F}}_{p}(\chi)\right)$ which were defined in Section 3 using the ramification filtration. Note that since $c_{\mathrm{un}} \in H_{\mathrm{un}}^{1}\left(G_{K}, \overline{\mathbf{F}}_{p}(\chi)\right)$ (if $\chi$ is trivial) and $c_{\mathrm{tr}} \notin H_{\mathrm{ty}}^{1}\left(G_{K}, \overline{\mathbf{F}}_{p}(\chi)\right)$ (if $\chi$ is cyclotomic), we always have that $\left\{c_{\tau} \mid \tau \in T\right\}$ is a basis for the $f$-dimensional space $H_{\mathrm{ty}}^{1}\left(G_{K}, \overline{\mathbf{F}}_{p}(\chi)\right) / H_{\text {un }}^{1}\left(G_{K}, \overline{\mathbf{F}}_{p}(\chi)\right)$.

THEOREM 6.6. With the above notation we have:

(1) If $\tau \in T$, then the following hold:

(a) $\{\tau\}$ is admissible if and only if $c_{\tau} \in H_{\mathrm{cg}}^{1}\left(G_{K}, \overline{\mathbf{F}}_{p}(\chi)\right)$;

(b) $T-\{\tau\}$ is admissible if and only if $c_{\tau} \notin H_{\mathrm{gt}}^{1}\left(G_{K}, \overline{\mathbf{F}}_{p}(\chi)\right)$.

(2) The following are equivalent:

(a) $\chi$ is generic;

(b) all subsets of $T$ are admissible;

(c) $H_{\mathrm{cg}}^{1}\left(G_{K}, \overline{\mathbf{F}}_{p}(\chi)\right)=H_{\mathrm{ty}}^{1}\left(G_{K}, \overline{\mathbf{F}}_{p}(\chi)\right)$;

(d) $H_{\mathrm{gt}}^{1}\left(G_{K}, \overline{\mathbf{F}}_{p}(\chi)\right)=H_{\mathrm{un}}^{1}\left(G_{K}, \overline{\mathbf{F}}_{p}(\chi)\right)$. 
Proof. To prove part (1), let $\tau=\tau_{i}$. From the proof of Theorem 5.1, we see that $c_{\tau}$ is in $\operatorname{Fil}^{s} H^{1}\left(G_{K}, \overline{\mathbf{F}}_{p}(\chi)\right)$ but not in $\operatorname{Fil}^{<s} H^{1}\left(G_{K}, \overline{\mathbf{F}}_{p}(\chi)\right)$, where $s=1+n_{i}^{\prime} / e$ and $n_{i}^{\prime}$ is as in the definition of the classes $u_{i}$.

For (1a), note that $\{i\}$ fails to be admissible if and only if $a_{i+1} \neq p$ and $\left(a_{j}, \ldots, a_{i}\right)=(p, p-1, \ldots, p-1)$ for some $j$ with $i-f+1<j \leqslant i$, which in turn is equivalent to $n_{i}^{\prime}>e$. Therefore, $\{\tau\}$ is admissible if and only if $c_{\tau} \in \operatorname{Fil}^{2} H^{1}\left(G_{K}, \overline{\mathbf{F}}_{p}(\chi)\right)=H_{\mathrm{cg}}^{1}\left(G_{K}, \overline{\mathbf{F}}_{p}(\chi)\right)$.

For (1b), note that $T-\{\tau\}$ is admissible if and only if $a_{i+1} \neq p$ or $\left.\chi\right|_{I_{K}}$ is not cyclotomic, which in turn is equivalent to $n_{i}^{\prime} \geqslant e /(p-1)$. Therefore, $T-\{\tau\}$ is admissible if and only if $c_{\tau} \notin \mathrm{Fil}^{<p /(p-1)} H^{1}\left(G_{K}, \overline{\mathbf{F}}_{p}(\chi)\right)=H_{\mathrm{gt}}^{1}\left(G_{K}, \overline{\mathbf{F}}_{p}(\chi)\right)$.

Turning to part (2), note that (2a) and (2b) are both equivalent to the condition that there be no dependent pairs, which in turn is equivalent to the admissibility of all singletons. The equivalence of (2b) and (2c) thus follows from (1a) and the fact that the $\left\{c_{\tau} \mid \tau \in T\right\}$ span $H_{\mathrm{ty}}^{1}\left(G_{K}, \overline{\mathbf{F}}_{p}(\chi)\right) / H_{\text {un }}^{1}\left(G_{K}, \overline{\mathbf{F}}_{p}(\chi)\right)$. The equivalence of $(2 \mathrm{a})$ and $(2 \mathrm{~d})$ is immediate from part (5) of Corollary 3.2.

\section{Distinguished subspaces}

We now return to the setting of Section 2, so $K$ is an unramified extension of $\mathbf{Q}_{p}$ of degree $f$ with residue field $k, T$ is the set of embeddings $k \rightarrow \overline{\mathbf{F}}_{p}$, and $\rho: G_{K} \rightarrow \mathrm{GL}_{2}\left(\overline{\mathbf{F}}_{p}\right)$ is a continuous representation. We assume further that $\rho$ is reducible, so $\rho \sim\left(\begin{array}{cc}\chi_{1} & * \\ 0 & \chi_{2}\end{array}\right)$ for some characters $\chi_{1}$ and $\chi_{2}$ of $G_{K}$. We let $\chi=\chi_{1} \chi_{2}^{-1}$ and let $c_{\rho} \in H^{1}\left(G_{K}, \overline{\mathbf{F}}_{p}(\chi)\right)$ denote the extension class associated to $\rho$.

Recall that we have defined a set $W^{\prime}\left(\chi_{1}, \chi_{2}\right)$ of certain pairs $(V, J)$, where $V$ is a Serre weight (that is, an irreducible representation of $\mathrm{GL}_{2}(k)$ over $\overline{\mathbf{F}}_{p}$ ) and $J$ is a subset of $T$ and for each $(V, J) \in W^{\prime}\left(\chi_{1}, \chi_{2}\right)$ a certain subspace $L_{V, J}$ of $H^{1}\left(G_{K}, \overline{\mathbf{F}}_{p}(\chi)\right)$. We then define $\mathrm{d} L_{V}$ as the union of the subspaces $L_{V, J}$ such that $(V, J) \in W^{\prime}\left(\chi_{1}, \chi_{2}\right)$, and $W(\rho)$ as the set of $V$ such that $c_{\rho} \in L_{V}$. In this section we give a conjectural description of $L_{V}$ in terms of the basis for $H^{1}\left(G_{K}, \overline{\mathbf{F}}_{p}(\chi)\right)$ of Corollary 5.2.

As in the preceding sections, we choose an embedding $\tau_{0} \in T$, set $\tau_{i}=\tau_{0}$ 。 Frob $^{i}$ (for $i \in \mathbf{Z}$ or $\mathbf{Z} / f \mathbf{Z}$ ), and let $\omega_{\tau_{i}}: I_{K} \rightarrow \overline{\mathbf{F}}_{p}^{\times}$denote the corresponding fundamental character. We let $\chi=\chi_{1} \chi_{2}^{-1}$ and write

$$
\left.\chi\right|_{I_{K}}=\prod_{i=0}^{f-1} \omega_{\tau_{i}}^{a_{i}},
$$

where $\left(a_{0}, \ldots, a_{f-1}\right)$ is the tame signature of $\chi$. We often interchange $\tau_{i}$ and $i$ in the notation, and thus identify $T$ with $\mathbf{Z} / f \mathbf{Z}$ (except in the notation for fundamental characters where this could lead to confusion). 
7.1. Shifting functions $\boldsymbol{\delta}$ and $\boldsymbol{\mu}$. For any subset $J$ of $\mathbf{Z} / f \mathbf{Z}$, we define a subset $\mu(J)$ of $\mathbf{Z} / f \mathbf{Z}$. First we define a function $\delta: \mathbf{Z} \rightarrow \mathbf{Z}$ depending on the integers $a_{i}$ as follows: If $j \in \mathbf{Z}$ we let $\delta(j)=j$ unless

$$
\left(a_{i+1}, a_{i+2}, \ldots, a_{j}\right)=(p, p-1, \ldots, p-1)
$$

for some $i<j$ (necessarily unique), in which case we let $\delta(j)=i$. Note that $\delta$ induces a function $\mathbf{Z} / f \mathbf{Z} \rightarrow \mathbf{Z} / f \mathbf{Z}$, which we also denote by $\delta$. If $\delta(J) \subset J$, then we let $\mu(J)=J$. Otherwise we choose some $\left[i_{1}\right] \in \delta(J) \backslash J$ and let $j_{1}$ be the least integer such that $j_{1}>i_{1},\left[j_{1}\right] \in J$ and $\delta\left(j_{1}\right)=i_{1}$. Now write $J=\left\{\left[j_{1}\right], \ldots,\left[j_{r}\right]\right\}$ with $j_{1}<j_{2}<\cdots<j_{r}<j_{1}+f$, and define $i_{\kappa}$ for $\kappa=2, \ldots, r$ inductively as follows:

$$
i_{\kappa}= \begin{cases}\delta\left(j_{\kappa}\right) & \text { if } i_{\kappa-1}<\delta\left(j_{\kappa}\right) \\ j_{\kappa} & \text { otherwise. }\end{cases}
$$

We then have $i_{1}<i_{2}<\cdots<i_{r}<i_{1}+f$, and we set $\mu(J)=\left\{\left[i_{1}\right], \ldots,\left[i_{r}\right]\right\}$. One checks easily that this is independent of the choice of $i_{1}$. Note that by construction we have $\delta(J) \subset \mu(J) \subset \delta(J) \cup J$.

LEMMA 7.1. The set $\mu(J)$ is admissible.

Proof. Suppose that $([i+t],[i])$ is a dependent pair with notation as in Definition 6.1. We must show that if $[i+t] \in \mu(J)$, then $[i] \in \mu(J)$. Recall from Section 6.1 that $s$ is such that $a_{i+s}=p-1$ but $a_{i+s+1}=p$. Note that

$\delta(i+1)=\cdots=\delta(i+s)=i$, and $\delta(i+v)=i+v-1$ for $v=s+1, \ldots, t$.

In particular $\delta([i+t]) \neq[i+t]$, and since $a_{i+t+1} \neq p$, it follows that $[i+t]$ is not in the image of $\delta$. If $[i+t] \in \mu(J)$, we must therefore have $i+t=j_{\kappa}=i_{\kappa}$ for some choice of $i_{1}$ and some $\kappa \in\{2, \ldots, r\}$. If $s<t$, then the resulting inequalities

$$
j_{\kappa}-1=\delta\left(j_{\kappa}\right) \leqslant i_{\kappa-1} \leqslant j_{\kappa-1}<j_{\kappa}
$$

imply that $i_{\kappa-1}=j_{\kappa-1}=i+t-1$ and $\kappa \geqslant 3$. Repeating the argument shows that for $v=2, \ldots, s-t$, we have $i_{\kappa-v}=j_{\kappa-v}=i+t-v$ and $\kappa \geqslant v+2$. In particular $[i+s] \in J$, and hence $[i]=\delta([i+s]) \in \mu(J)$.

7.2. Explicit distinguished subspaces. Now let $V=V_{\vec{d}, \vec{b}}$ and suppose that $(V, J) \in W^{\prime}\left(\chi_{1}, \chi_{2}\right)$ for some $J \subset T$. Then there is a unique $J_{\max } \subset \mathbf{Z} / f \mathbf{Z}$ such that $\left(V, J_{\max }\right) \in W^{\prime}\left(\chi_{1}, \chi_{2}\right)$, and $J_{\max }$ satisfies the two conditions:

- if $\left(b_{i}, b_{i+1}, \ldots, b_{j-1}, b_{j}\right)=(p, p-1, \ldots, p-1,1)$ for some $i<j$ such that $i, i+1, \ldots, j-1 \notin J_{\max }$, then $j \notin J_{\max }$; 
- if $\left(b_{0}, \ldots, b_{f-1}\right)=(p-1, p-1, \ldots, p-1)$, or if $p=2$ and $\left(b_{0}, \ldots, b_{f-1}\right)=$ $(2,2, \ldots, 2)$, then $J_{\max } \neq \emptyset$.

(This is proved in [15] for $p>2$, but one easily checks that it holds also for $p=2$.) We then define $L_{V}^{\mathrm{AH}} \subset H^{1}\left(G_{K}, \overline{\mathbf{F}}_{p}(\chi)\right)$ to be the span of $\left\{c_{\tau} \mid \tau \in \mu\left(J_{\max }\right)\right\}$ together with $c_{\text {un }}$ if $\chi$ is trivial, unless $\chi$ is cyclotomic and $V=V_{\vec{d}, \vec{b}}$ with $J_{\max }=T$ and $\left(b_{0}, \ldots, b_{f-1}\right)=(p, p, \ldots, p)$, in which case $L_{V}^{\mathrm{AH}}=H^{1}\left(G_{K}, \overline{\mathbf{F}}_{p}(\chi)\right)$ (that is, we include $c_{\text {tr }}$ as well). By Corollary 6.5 and Lemma 7.1 the space $L_{V}^{\mathrm{AH}}$ is well defined, that is, independent of the choices made in Section 5. (The superscript $\mathrm{AH}$ refers to the use of the Artin-Hasse exponential in its definition.)

We now state our conjectural explicit description of the subspaces appearing in the recipe for the weight:

Conjecture 7.2. If $(V, J) \in W^{\prime}\left(\chi_{1}, \chi_{2}\right)$ for some $J$, then $L_{V}=L_{V}^{\mathrm{AH}}$.

Recall that [15] proves that if $p>2$ and $(V, J) \in W^{\prime}\left(\chi_{1}, \chi_{2}\right)$, then $L_{V, J} \subset L_{V, J_{\max }}$, so that $L_{V}$ can be replaced by $L_{V, J_{\max }}$ in the statement of Conjecture 7.2 if $p>2$. Since $L_{V}^{\mathrm{AH}}$ is a subspace of $H^{1}\left(G_{K}, \overline{\mathbf{F}}_{p}(\chi)\right)$ of dimension at most that of $L_{V, J_{\max }}$, Conjecture 7.2 implies that $L_{V}^{\mathrm{AH}}=L_{V, J_{\max }}$, and hence the assertion that $L_{V, J} \subset L_{V, J_{\max }}$ for all $(V, J) \in W^{\prime}\left(\chi_{1}, \chi_{2}\right)$ still holds for $p=2$.

7.3. A weight-explicit Serre's conjecture. Finally we record a more explicit form of Conjecture 2.1. For $\rho$ as above, define $W^{\mathrm{AH}}(\rho)$ to be the set of $V$ such that $(V, J) \in W^{\prime}\left(\chi_{1}, \chi_{2}\right)$ for some $J$ and $c_{\rho} \in L_{V}^{\mathrm{AH}}$. For irreducible $\rho: G_{K} \rightarrow$ $\mathrm{GL}_{2}\left(\overline{\mathbf{F}}_{p}\right)$, define $W^{\mathrm{AH}}(\rho)=W(\rho)$.

Suppose now that $\rho: G_{F} \rightarrow \mathrm{GL}_{2}\left(\overline{\mathbf{F}}_{p}\right)$ is continuous, irreducible and totally odd. Combining Conjectures 2.1 and 7.2 then yields:

CONJECTURE 7.3. The representation $\rho$ is modular of weight $V=\otimes_{\left\{\mathfrak{p} \in S_{p}\right\}} V_{\mathfrak{p}}$ if and only if $V_{\mathfrak{p}} \in W^{\mathrm{AH}}\left(\left.\rho\right|_{G_{F_{\mathfrak{p}}}}\right)$ for all $\mathfrak{p} \in S_{p}$.

If $\rho$ is modular (of some weight) and satisfies the hypotheses under which the weight part of Serre's Conjecture is known (by [14] or [20]), then Conjecture 7.3 is immediate from Conjecture 7.2.

\section{The quadratic case}

In this section we delineate the possibilities for the spaces of extensions $L_{V}^{\mathrm{AH}}$ and the sets of Serre weights $W^{\mathrm{AH}}(\rho)$ in the case $f=2$. We refer the reader to the forthcoming paper [9] for a more detailed discussion of the situation for arbitrary $f$ and the underlying combinatorics. 
Suppose now that $K$ is the unramified quadratic extension of $\mathbf{Q}_{p}$, and $\rho: G_{K} \rightarrow$ $\mathrm{GL}_{2}\left(\overline{\mathbf{F}}_{p}\right)$ is a continuous representation.

8.1. Three reducible cases. Suppose first that $\rho$ is reducible, so that $\rho \sim\left(\begin{array}{cc}\chi_{1} & * \\ 0 & \chi_{2}\end{array}\right)$ for some characters $\chi_{1}, \chi_{2}: G_{K} \rightarrow \overline{\mathbf{F}}_{p}^{\times}$. Twisting by $\chi_{2}^{-1}$, we may assume $\chi_{2}=1$, and we write $\chi$ for $\chi_{1}$ and $c_{\rho} \in H^{1}\left(G_{K}, \overline{\mathbf{F}}_{p}(\chi)\right)$ for the associated extension class. Choosing an embedding $\tau_{0}: k \rightarrow \overline{\mathbf{F}}_{p}$, we may write $\left.\chi\right|_{I_{K}}=\omega_{\tau_{0}}^{a}$ with $p+1 \leqslant a<p^{2}+p$. We let $\left(a_{0}, a_{1}\right)$ denote the tame signature of $\chi$, so $a=a_{0}+a_{1} p$; altering our choice of $\tau_{0}$, we may further assume that $1 \leqslant a_{0} \leqslant p-1$ and $a_{0} \leqslant a_{1} \leqslant p$. We now divide our analysis into three cases, following the terminology introduced after Theorem 3.1:

(I) $\chi$ is primitive and generic: $1 \leqslant a_{0}<a_{1}<p$;

(II) $\chi$ is imprimitive and generic: $1 \leqslant a_{0}=a_{1}<p$;

(III) $\chi$ is primitive and nongeneric: $1 \leqslant a_{0}<a_{1}=p$.

Thus the analysis in Case I is simplest, and the other two cases represent the two main complications that can occur. Note that Case II occurs precisely when $\chi$ has absolute niveau 1 , so $\left.\chi\right|_{I_{K}}=\omega^{c}$ where $\omega$ is the cyclotomic character and $1 \leqslant c \leqslant p-1$, and Case III occurs precisely when $\left.\chi\right|_{I_{K}}=\omega_{\tau}^{c}$ for some $\tau$ and $c$ with $1 \leqslant c \leqslant p-1$. Note also for $f=2$ (or indeed any prime $f$ ), $\chi$ cannot be both imprimitive and nongeneric.

8.2. Case I. In Case I, the elements of $W^{\prime}(\chi, 1)$ are the pairs $\left(V_{\vec{d}, \vec{b}}, J\right)$ given by the columns of the table:

\begin{tabular}{ccccc}
\hline$J$ & $T$ & $\{0\}$ & $\{1\}$ & $\emptyset$ \\
\hline$\vec{d}$ & $(0,0)$ & $\left(p-1, a_{1}-1\right)$ & $\left(a_{0}-1, p-1\right)$ & $\left(a_{0}, a_{1}\right)$ \\
$\vec{b}$ & $\left(a_{0}, a_{1}\right)$ & $\left(a_{0}+1, p-a_{1}\right)$ & $\left(p-a_{0}, a_{1}+1\right)$ & $\left(b_{0}, b_{1}\right)$ \\
\hline
\end{tabular}

where

$$
\left(b_{0}, b_{1}\right)= \begin{cases}\left(p-1-a_{0}, p-1-a_{1}\right) & \text { if } a_{1}<p-1 \\ \left(p-2-a_{0}, p\right) & \text { if } a_{1}=p-1 \text { and } a_{0}<p-2 \\ (p, p-1) & \text { if }\left(a_{0}, a_{1}\right)=(p-2, p-1)\end{cases}
$$

For each $V=V_{\vec{d}, \vec{b}}$ in the table, there is a unique $J \in S_{V}(\chi, 1)$, so that $J=J_{\max }$; moreover, $J=\mu(J)$ is admissible. If $J=T$, then $L_{V}^{\mathrm{AH}}$ is the whole twodimensional space $H^{1}\left(G_{K}, \overline{\mathbf{F}}_{p}(\chi)\right)$, and if $J=\emptyset$, then $L_{V}^{\mathrm{AH}}=0$, but if $J=\{i\}$ 
for $i=0$ or 1 , then $L_{V}^{\mathrm{AH}}$ is a one-dimensional subspace of $H^{1}\left(G_{K}, \overline{\mathbf{F}}_{p}(\chi)\right)$ which we simply denote $L_{i}$. We then have four possibilities for $W^{\mathrm{AH}}(\rho)$ :

(Ia) $\left\{V_{(0,0),\left(a_{0}, a_{1}\right)}\right\}$ if $c_{\rho} \notin L_{0} \cup L_{1}$;

$\left(\mathrm{Ib}_{1}\right)\left\{V_{(0,0),\left(a_{0}, a_{1}\right)}, V_{\left(a_{0}-1, p-1\right),\left(p-a_{0}, a_{1}+1\right)}\right\}$ if $c_{\rho} \in L_{1}, c_{\rho} \neq 0$;

$\left(\mathrm{Ib}_{2}\right)\left\{V_{(0,0),\left(a_{0}, a_{1}\right)}, V_{\left(p-1, a_{1}-1\right),\left(a_{0}+1, p-a_{1}\right)}\right\}$ if $c_{\rho} \in L_{0}, c_{\rho} \neq 0$;

(Ic) $\left\{V_{(0,0),\left(a_{0}, a_{1}\right)}, V_{\left(p-1, a_{1}-1\right),\left(a_{0}+1, p-a_{1}\right)}, V_{\left(a_{0}-1, p-1\right),\left(p-a_{0}, a_{1}+1\right)}, V_{\left(a_{0}, a_{1}\right),\left(b_{0}, b_{1}\right)}\right\}$ if $c_{\rho}=0$.

We now proceed to describe the subspaces $L_{0}$ and $L_{1}$.

With notation as in Section 5, we have $n_{0}=a=a_{0}+p a_{1}$ and $n_{1}=a_{1}+p a_{0}$, so that $n_{1}<n_{0}$. Choosing a tamely ramified extension $M$ with uniformizer $\pi$, residue field $l$, and ramification degree $e$ as in that section, we have $n_{0}^{\prime}=e n_{1} /\left(p^{2}-1\right)$, $n_{1}^{\prime}=e n_{0} /\left(p^{2}-1\right)$, and

$$
\chi=\mu\left(\tau_{1} \circ \bar{\omega}_{\pi}\right)^{n_{0}^{\prime}}=\mu\left(\tau_{0} \circ \bar{\omega}_{\pi}\right)^{n_{1}^{\prime}},
$$

where $\omega_{\pi}(g)=g(\pi) / \pi$.

Theorem 5.1 provides a basis $\left\{u_{0}, u_{1}\right\}$ for $U_{\chi}=\left(M^{\times} \otimes \overline{\mathbf{F}}_{p}\left(\chi^{-1}\right)\right)^{\mathrm{Gal}(M / K)}$ with

$$
u_{i} \in \varepsilon_{\pi_{i}^{n_{i}^{\prime}}}\left(l \otimes \overline{\mathbf{F}}_{p}\right)=E\left([l] \pi^{n_{i}^{\prime}}\right) \otimes \overline{\mathbf{F}}_{p} .
$$

We can therefore describe the elements of the dual basis $\left\{c_{0}, c_{1}\right\}$ for

$$
H^{1}\left(G_{K}, \overline{\mathbf{F}}_{p}(\chi)\right) \cong \operatorname{Hom}_{\mathrm{Gal}(M / K)}\left(M^{\times}, \overline{\mathbf{F}}_{p}(\chi)\right)
$$

by specifying their values on the elements of $M^{\times}$of the form $E\left([a] \pi^{n_{i}^{\prime}}\right)$ for $a \in l$ and $i=0,1$. We find that $c_{0}$ and $c_{1}$ are defined (up to scalars) by the homomorphisms

$$
c_{0}\left(E\left([a] \pi^{n_{1}^{\prime}}\right)\right)=0, \quad c_{0}\left(E\left([a] \pi^{n_{0}^{\prime}}\right)\right)=\sum_{g \in \operatorname{Gal}(l / k)} \mu^{-1}(g) \tilde{\tau}_{0}\left(g a^{p}\right)
$$

and $\quad c_{1}\left(E\left([a] \pi^{n_{0}^{\prime}}\right)\right)=0, \quad c_{1}\left(E\left([a] \pi^{n_{1}^{\prime}}\right)\right)=\sum_{g \in \operatorname{Gal}(l / k)} \mu^{-1}(g) \tilde{\tau}_{1}\left(g a^{p}\right)$

for any choices of embeddings $\tilde{\tau}_{i}: l \rightarrow \overline{\mathbf{F}}_{p}$ extending the $\tau_{i}$. Indeed it is straightforward to check that $c_{i}(h x)=\chi(h) c_{0}(x)$ for $x=E\left([a] \pi^{n_{i}^{\prime}}\right)$, we clearly have $c_{0}\left(u_{1}\right)=c_{1}\left(u_{0}\right)=0$, and the following lemma shows that $c_{0}$ and $c_{1}$ are not identically 0 . For the lemma, we momentarily drop the assumptions that $\left[k: \mathbf{F}_{p}\right]=2$ and that $[l: k]$ is not divisible by $p$. 
LEMMA 8.1. Suppose that $k \subset l$ are finite extensions of $\mathbf{F}_{p}, \mu: \operatorname{Gal}(l / k) \rightarrow \overline{\mathbf{F}}_{p}^{\times}$ is a character and $\tilde{\tau}: l \rightarrow \overline{\mathbf{F}}_{p}$ is an embedding. Then the function $f: l \rightarrow \overline{\mathbf{F}}_{p}$ defined by $f(a)=\sum_{g \in \operatorname{Gal}(l / k)} \mu(g) \tilde{\tau}(g a)$ is not identically zero.

Proof. Suppose that $f(a)=0$ for all $a \in l$. Let $\mathbf{F}$ denote the subfield of $\overline{\mathbf{F}}_{p}$ generated by the values of $\mu$, and let $r=\left[\mathbf{F}: \mathbf{F}_{p}\right]$. For $i=0, \ldots, r-1$, consider the function $f^{(i)}: l \rightarrow \overline{\mathbf{F}}_{p}$ defined by $f^{(i)}(a)=\sum_{g \in \operatorname{Gal}(l / k)} \mu^{p^{i}}(g) \tilde{\tau}(g a)$. Since $f^{(i)}\left(a^{p^{i}}\right)=(f(a))^{p^{i}}=0$ for all $a \in l$, the function $f^{(i)}$ is identically zero, and therefore so is the function $h: l \rightarrow \overline{\mathbf{F}}_{p}$ defined by

$$
h(a)=\sum_{i=0}^{r-1} f^{(i)}(a)=\sum_{g \in \operatorname{Gal}(l / k)} \operatorname{tr}_{\mathbf{F} / \mathbf{F}_{p}}(\mu(g)) \tilde{\tau}(g a) .
$$

Taking $a$ so that $\{g a \mid g \in \operatorname{Gal}(l / k)\}$ is a normal basis for $l / k$, the $\tilde{\tau}(g a)$ are linearly independent over $\tilde{\tau}(k)$, and hence over $\mathbf{F}_{p}$. It follows that $\operatorname{tr}_{\mathbf{F} / \mathbf{F}_{p}}(\mu(g))=0$ for all $g \in \operatorname{Gal}(l / k)$. Since the values $\mu(g)$ span $\mathbf{F}$ as a vector space over $\mathbf{F}_{p}$, this implies that $\operatorname{tr}_{\mathbf{F} / \mathbf{F}_{p}}$ is identically zero, yielding a contradiction.

We thus obtain the criterion that $c_{\rho} \in L_{i}$ if and only if $E\left([a] \pi^{e n_{i} /\left(p^{2}-1\right)}\right) \in$ $\operatorname{ker}\left(c_{\rho}\right)$ for all $a \in l$. Since $n_{1}<n_{0}$, this provides a description of $L_{0}$ in terms of the ramification filtration on cohomology defined in Section 3. By Theorem 3.1, we have

$$
\operatorname{dim}_{\overline{\mathbf{F}}_{p}} \operatorname{Fil}^{s}\left(H^{1}\left(G_{K}, \overline{\mathbf{F}}_{p}(\chi)\right)\right)= \begin{cases}0 & \text { if } s<1+n_{1} /\left(p^{2}-1\right), \\ 1 & \text { if } 1+n_{1} /\left(p^{2}-1\right) \leqslant s<1+n_{0} /\left(p^{2}-1\right), \\ 2 & \text { if } 1+n_{0} /\left(p^{2}-1\right) \leqslant s .\end{cases}
$$

We thus see that

$$
L_{0}=\operatorname{Fil}^{1+n_{1} /\left(p^{2}-1\right)}\left(H^{1}\left(G_{K}, \overline{\mathbf{F}}_{p}(\chi)\right)\right)=\operatorname{Fil}^{<1+n_{0} /\left(p^{2}-1\right)}\left(H^{1}\left(G_{K}, \overline{\mathbf{F}}_{p}(\chi)\right)\right),
$$

so that $c_{\rho} \in L_{0}$ if and only if $G_{K}^{n_{0} /\left(p^{2}-1\right)} \subset \operatorname{ker}(\rho)$. The space $L_{1}$ cannot be described in terms of the ramification filtration, but it can still be characterized in terms of splitting fields. Indeed if we let $N$ denote the splitting field over $M$ of $\rho$, then we have

$$
c_{\rho} \in L_{i} \quad \text { if and only if } \quad E\left([a] \pi^{e n_{i} /\left(p^{2}-1\right)}\right) \in \operatorname{Norm}_{N / M}\left(N^{\times}\right) \text {for all } a \in l .
$$

8.3. Case II. We now turn to Case II, where the tame signature $\left(a_{0}, a_{0}\right)$ has period 1 . Then $W^{\prime}(\chi, 1)$ is given exactly as in (10) with the following changes: 
- if $a_{0}=1$, then we also have $\vec{d}=(0,0), \vec{b}=(p, p)$ for $J=T$;

- if $a_{0}=p-2$, then we also have $\vec{d}=(p-2, p-2), \vec{b}=(p, p)$ for $J=\emptyset$;

- if $a_{0}=p-1$, then take $\vec{b}=(p-1, p-1)$ for $J=\emptyset$, and we have the following additional elements:

\begin{tabular}{cccc}
\hline$J$ & $\{0\}$ & $\{1\}$ & $\emptyset$ (if $p=2)$ \\
\hline$\vec{d}$ & $(p-2, p-1)$ & $(p-1, p-2)$ & $(0,0)$ \\
$\vec{b}$ & $(1, p)$ & $(p, 1)$ & $(2,2)$ \\
\hline
\end{tabular}

For each $V$ we still have a unique $J \in S_{V}(\chi, 1)$ unless $a_{0}=p-1$, in which case each $S_{V}(\chi, 1)$ has two elements, and the ones appearing in the last bullet above are precisely those for which $J \neq J_{\max }$. Note that every $J$ arises as $J_{\max }$ for some $V$ unless $a_{0}=p-1$, in which case $J_{\max }=\varnothing$ does not arise. Moreover, $J_{\max }$ uniquely determines $V$ unless $a_{0}=1$, in which case $V_{(0,0),(1,1)}$ and $V_{(0,0),(p, p)}$ both have $J_{\max }=T$, or $a_{0}=p-2$, in which case $V_{(p-2, p-2),(0,0)}$ and $V_{(p-2, p-2),(p, p)}$ both have $J_{\max }=\emptyset$. It is still the case that $J=\mu(J)$ is admissible for every $J$.

If $J_{\max }=\emptyset$, then $L_{V}^{\mathrm{AH}}=0$. If $J_{\max }=T$, then $L_{V}^{\mathrm{AH}}=H^{1}\left(G_{K}, \overline{\mathbf{F}}_{p}(\chi)\right)$ unless $\chi$ is cyclotomic and $V=V_{(0,0),(1,1)}$, in which case $L_{V}^{\mathrm{AH}}=H_{\mathrm{fl}}^{1}\left(G_{K}, \overline{\mathbf{F}}_{p}(\chi)\right)=$ $H_{\mathrm{ty}}^{1}\left(G_{K}, \overline{\mathbf{F}}_{p}(\chi)\right.$ has codimension one in $H^{1}\left(G_{K}, \overline{\mathbf{F}}_{p}(\chi)\right)$. If $J_{\max }=\{i\}$ for $i=0$ or 1 , then writing simply $L_{i}$ for $L_{V}^{\mathrm{AH}}$, we have the sequence of inclusions of subspaces with codimension one:

$$
H_{\mathrm{un}}^{1}\left(G_{K}, \overline{\mathbf{F}}_{p}(\chi)\right) \subset L_{i} \subset H_{\mathrm{ty}}^{1}\left(G_{K}, \overline{\mathbf{F}}_{p}(\chi)\right) .
$$

We now list the possibilities for $W^{\mathrm{AH}}(\rho)$.

If $a_{0}=1$, then $W^{\mathrm{AH}}(\rho)$ is:

(IIz) $\left\{V_{(0,0),(p, p)}\right\}$ if $c_{\rho} \notin H_{\mathrm{ty}}^{1}\left(G_{K}, \overline{\mathbf{F}}_{p}(\chi)\right)$;

(IIa) $\left\{V_{(0,0),(p, p)}, V_{(0,0),(1,1)}\right\}$ if $c_{\rho} \in H_{\mathrm{ty}}^{1}\left(G_{K}, \overline{\mathbf{F}}_{p}(\chi)\right)-\left(L_{0} \cup L_{1}\right)$;

$\left(\mathrm{IIb}_{1}\right)\left\{V_{(0,0),(p, p)}, V_{(0,0),(1,1)}, V_{(0, p-1),(p-1,2)}\right\}$ if $c_{\rho} \in L_{1}-L_{0}$;

$\left(\mathrm{IIb}_{2}\right)\left\{V_{(0,0),(p, p)}, V_{(0,0),(1,1)}, V_{(p-1,0),(2, p-1)}\right\}$ if $c_{\rho} \in L_{0}-L_{1}$;

(IIc) $\left\{V_{(0,0),(p, p)}, V_{(0,0),(1,1)}, V_{(p-1,0),(2, p-1)}, V_{(0, p-1),(p-1,2)}, V_{(1,1),(p-2, p-2)}\right\}$ if $c_{\rho} \in L_{0} \cap L_{1}=H_{\text {un }}^{1}\left(G_{K}, \overline{\mathbf{F}}_{p}(\chi)\right)$,

where in Case (IIc) we omit $V_{(1,1),(p-2, p-2)}$ if $p=2$ and add $V_{(1,1),(3,3)}$ if $p=3$. Note that Case (IIz) is only possible if $\chi$ is cyclotomic, and recall that $H_{\mathrm{un}}^{1}\left(G_{K}, \overline{\mathbf{F}}_{p}(\chi)\right)=0$ unless $\chi$ is trivial (which implies here that $p=2$ ). 
If $2 \leqslant a_{0} \leqslant p-1$, then $W^{\mathrm{AH}}(\rho)$ is:

(IIa') $\left\{V_{(0,0),\left(a_{0}, a_{0}\right)}\right\}$ if $c_{\rho} \notin L_{0} \cup L_{1}$;

$\left(\mathrm{IIb}_{1}^{\prime}\right)\left\{V_{(0,0),\left(a_{0}, a_{0}\right)}, V_{\left(a_{0}-1, p-1\right),\left(p-a_{0}, a_{0}+1\right)}\right\}$ if $c_{\rho} \in L_{1}-L_{0}$;

$\left(\mathrm{IIb}_{2}^{\prime}\right)\left\{V_{(0,0),\left(a_{0}, a_{0}\right)}, V_{\left(p-1, a_{0}-1\right),\left(a_{0}+1, p-a_{0}\right)}\right\}$ if $c_{\rho} \in L_{0}-L_{1}$;

$\left(\mathrm{IIc}^{\prime}\right)\left\{V_{(0,0),\left(a_{0}, a_{0}\right)}, V_{\left(p-1, a_{0}-1\right),\left(a_{0}+1, p-a_{0}\right)}, V_{\left(a_{0}-1, p-1\right),\left(p-a_{0}, a_{0}+1\right)}\right.$,

$$
\left.V_{\left(a_{0}, a_{0}\right),\left(p-1-a_{0}, p-1-a_{0}\right)}\right\} \text { if } c_{\rho} \in L_{0} \cap L_{1}=H_{\text {un }}^{1}\left(G_{K}, \overline{\mathbf{F}}_{p}(\chi)\right),
$$

where in Case (IIc') we omit $V_{(1,1),\left(p-1-a_{0}, p-1-a_{0}\right)}$ if $a_{0}=p-1$ and add $V_{(1,1),(p, p)}$ if $a_{0}=p-2$. (Recall again that $H_{\mathrm{un}}^{1}\left(G_{K}, \overline{\mathbf{F}}_{p}(\chi)\right)=0$ unless $\chi$ is trivial, in which case $a_{0}=p-1$.)

We now turn to the description of the subspaces $L_{i}$. The main difference from Case I is that we now have $n_{0}=n_{1}=a=a_{0}(1+p)$, so that $n_{0}^{\prime}=n_{1}^{\prime}$ in the notation of Section 5. (Note also that we may choose $e$ to divide $p-1$.) Another difference is that $\chi$ may be trivial or cyclotomic, so that $H^{1}\left(G_{K}, \overline{\mathbf{F}}_{p}(\chi)\right)$ and $U_{\chi}$ may have dimension greater than two. However, from the inclusions (13) we see that it suffices to describe the image $L_{i}^{\prime}$ of $L_{i}$ in the quotient

$$
\begin{aligned}
H_{\mathrm{ty}}^{1}\left(G_{K}, \overline{\mathbf{F}}_{p}(\chi)\right) / H_{\mathrm{un}}^{1}\left(G_{K}, \overline{\mathbf{F}}_{p}(\chi)\right) & \cong \operatorname{Hom}_{\mathrm{Gal}(M / K)}\left(\mathcal{O}_{M}^{\times} / U_{m}, \overline{\mathbf{F}}_{p}(\chi)\right) \\
& \subset \operatorname{Hom}_{\mathrm{Gal}(M / K)}\left(\mathcal{O}_{M}^{\times}, \overline{\mathbf{F}}_{p}(\chi)\right),
\end{aligned}
$$

where $U_{m}=1+\pi^{m} \mathcal{O}_{M}$ for $m=\lceil e p /(p-1)\rceil$. This quotient has a basis $\left\{c_{0}^{\prime}, c_{1}^{\prime}\right\}$ where $c_{i}^{\prime}$ spans $L_{i}^{\prime}$ and is determined by its values on elements of the form $E\left([a] \pi^{n_{0}^{\prime}}\right)$ for $a \in l$ by the formula

$$
c_{i}^{\prime}\left([a] \pi^{n_{0}^{\prime}}\right)=\sum_{g \in \operatorname{Gal}(l / k)} \mu^{-1}(g) \tilde{\tau}_{i}\left(g a^{p}\right),
$$

where $\tilde{\tau}_{i}: l \rightarrow \overline{\mathbf{F}}_{p}$ is any choice of embedding extending $\tau_{i}$. Indeed it follows from the definitions of the elements $u_{j}$ that $c_{i}^{\prime} \in L_{i}^{\prime}$ and from Lemma 8.1 that $c_{i}^{\prime} \neq 0$.

As for the ramification filtration on cohomology, the fact that the tame signature has period 1 in this case gives that

$$
\begin{aligned}
& \operatorname{dim}_{\overline{\mathbf{F}}_{p}} \operatorname{Fil}^{s}\left(H^{1}\left(G_{K}, \overline{\mathbf{F}}_{p}(\chi)\right)\right) \\
& \quad= \begin{cases}0 & \text { if } s<0, \\
\delta_{\text {triv }} & \text { if } 0 \leqslant s<1+n_{0} /\left(p^{2}-1\right), \\
\delta_{\text {triv }}+2 & \text { if } 1+n_{0} /\left(p^{2}-1\right) \leqslant s<1+p /(p-1), \\
\delta_{\text {triv }}+2+\delta_{\text {cyc }} & \text { if } 1+p /(p-1)) \leqslant s,\end{cases}
\end{aligned}
$$

where $\delta_{\text {triv }}$ (respectively $\delta_{\text {cyc }}$ ) is 1 or 0 according to whether or not $\chi$ is trivial (respectively cyclotomic). Unlike Case I, neither of the spaces $L_{i}$ can be described in terms of the ramification filtration, nor can we necessarily detect whether $c_{\rho} \in L_{i}$ from the splitting field of $\rho$. 
8.4. Case III. Finally we consider Case III, where the tame signature of $\chi$ has the form $\left(a_{0}, p\right)$. The elements of $W^{\prime}(\chi, 1)$ are then given in the table:

\begin{tabular}{cccccc}
\hline & $J$ & $T$ & $\{0\}$ & $\{1\}$ & $\emptyset$ \\
\hline \multirow{2}{*}{$1 \leqslant a_{0}<p-1$} & $\vec{d}$ & $(0,0)$ & $(p-2, p-1)$ & $\left(a_{0}, p-1\right)$ & $\left(a_{0}, p\right)$ \\
& $\vec{b}$ & $\left(a_{0}, p\right)$ & $\left(a_{0}+2, p\right)$ & $\left(p-1-a_{0}, 1\right)$ & $\left(b_{0}, b_{1}\right)$ \\
\hline \multirow{2}{*}{$a_{0}=p-1$} & $\vec{d}$ & $(0,0)$ & $(p-1,0)$ & $(p-1, p-2)$ & $(0,1)$ \\
& $\vec{b}$ & $(p-1, p)$ & $(1, p-1)$ & $(p, 2)$ & $\left(b_{0}, b_{1}\right)$ \\
\hline
\end{tabular}

where

$$
\left(b_{0}, b_{1}\right)= \begin{cases}\left(p-2-a_{0}, p-1\right) & \text { if } a_{0}<p-2 \\ (p, p-1) & \text { if } a_{0}=p-2 \text { or } p=2 \\ (p-1, p-2) & \text { if } a_{0}=p-1 \text { and } p>2\end{cases}
$$

As in Case I, there is a unique $J \in S_{V}(\chi, 1)$ for each $V$ in the table, so that $J=J_{\max }$. However, only $T,\{0\}$ and $\varnothing$ are admissible, and the functions $\delta$ and $\mu$ introduced in Section 7 are nontrivial. Indeed we find that $\mu(T)=T, \mu(\{0\})=$ $\mu(\{1\})=\{0\}$ and $\mu(\emptyset)=\emptyset$. If $J=T$, then $L_{V}^{\mathrm{AH}}$ is the whole two-dimensional space $H^{1}\left(G_{K}, \overline{\mathbf{F}}_{p}(\chi)\right)$, and if $J=\emptyset$, then $L_{V}^{\mathrm{AH}}=0$, but if $J=\{i\}$ for $i=0$ or 1 , then $L_{V}^{\mathrm{AH}}$ is the same one-dimensional subspace of $H^{1}\left(G_{K}, \overline{\mathbf{F}}_{p}(\chi)\right)$ which we simply denote $L_{0}$. We therefore have three possibilities for $W^{\mathrm{AH}}(\rho)$.

If $1 \leqslant a_{0}<p-1$, then $W^{\mathrm{AH}}(\rho)$ is:

(IIIa) $\left\{V_{(0,0),\left(a_{0}, p\right)}\right\}$ if $c_{\rho} \notin L_{0}$;

$\left(\mathrm{IIIb}_{1}\right)\left\{V_{(0,0),\left(a_{0}, p\right)}, V_{(p-2, p-1),\left(a_{0}+2, p\right)}, V_{\left(a_{0}, p-1\right),\left(p-1-a_{0}, 1\right)}\right\}$ if $c_{\rho} \in L_{0}, c_{\rho} \neq 0$;

(IIIC) $\left\{V_{(0,0),\left(a_{0}, p\right)}, V_{(p-2, p-1),\left(a_{0}+2, p\right)}, V_{\left(a_{0}, p-1\right),\left(p-1-a_{0}, 1\right)}, V_{\left(a_{0}, p\right),\left(b_{0}, b_{1}\right)}\right\}$ if $c_{\rho}=0$.

If $a_{0}=p-1$, then $W^{\mathrm{AH}}(\rho)$ is:

(IIIa') $\left\{V_{(0,0),\left(a_{0}, p\right)}\right\}$ if $c_{\rho} \notin L_{0}$;

$\left(\mathrm{IIIb}_{1}^{\prime}\right)\left\{V_{(0,0),\left(a_{0}, p\right)}, V_{(p-1,0),(1, p-1)}, V_{(p-1, p-2),(p, 2)}\right\}$ if $c_{\rho} \in L_{0}, c_{\rho} \neq 0$;

$\left(\mathrm{IIIc}^{\prime}\right)\left\{V_{(0,0),\left(a_{0}, p\right)}, V_{(p-1,0),(1, p-1)}, V_{(p-1, p-2),(p, 2)}, V_{(0,1),\left(b_{0}, b_{1}\right)}\right\}$ if $c_{\rho}=0$.

Turning to the subspace $L_{0}$, we now have $n_{0}=a_{0}+p^{2}$ and $n_{1}=\left(a_{0}+1\right) p$, so that again $n_{1}^{\prime}=e n_{0} /\left(p^{2}-1\right)$, but now $n_{0}^{\prime}=n_{1}^{\prime}-e$ if $a_{0}<p-1$ and $n_{0}^{\prime}=e /\left(p^{2}-1\right)=1$ if $a_{0}=p-1$. Therefore, $L_{0}$ is spanned by the class $c_{0}$ determined by the formula

$$
c_{0}\left(E\left([a] \pi^{n_{1}^{\prime}}\right)\right)=0, \quad c_{0}\left(E\left([a] \pi^{n_{0}^{\prime}}\right)\right)=\sum_{g \in \operatorname{Gal}(l / k)} \mu^{-1}(g) \tilde{\tau}_{0}\left(g a^{p}\right)
$$


for $a \in l$, where $\tilde{\tau}_{0}$ is any choice of embedding extending $\tau_{0}$. We thus obtain the criterion that $c_{\rho} \in L_{0}$ if and only if $E\left([a] \pi^{e n_{0} /\left(p^{2}-1\right)}\right) \in \operatorname{ker}\left(c_{\rho}\right)$ for all $a \in l$. In terms of the splitting field $N$ of $\rho$ over $M$, we have

$c_{\rho} \in L_{0} \quad$ if and only if $\quad E\left([a] \pi^{e n_{0} /\left(p^{2}-1\right)}\right) \in \operatorname{Norm}_{N / M}\left(N^{\times}\right)$for all $a \in l$.

As for the ramification filtration, we now have

$\operatorname{dim}_{\overline{\mathbf{F}}_{p}} \operatorname{Fil}^{s}\left(H^{1}\left(G_{K}, \overline{\mathbf{F}}_{p}(\chi)\right)\right)= \begin{cases}0 & \text { if } s<1+m /\left(p^{2}-1\right), \\ 1 & \text { if } 1+m /\left(p^{2}-1\right) \leqslant s<1+n_{0} /\left(p^{2}-1\right), \\ 2 & \text { if } 1+n_{0} /\left(p^{2}-1\right) \leqslant s,\end{cases}$ where $m=a_{0}+1$ if $1 \leqslant a_{0}<p-1$ and $m=1$ if $a_{0}=p-1$. We thus see that

$$
L_{0}=\mathrm{Fil}^{1+m /\left(p^{2}-1\right)}\left(H^{1}\left(G_{K}, \overline{\mathbf{F}}_{p}(\chi)\right)\right)=\mathrm{Fil}^{<1+n_{0} /\left(p^{2}-1\right)}\left(H^{1}\left(G_{K}, \overline{\mathbf{F}}_{p}(\chi)\right)\right),
$$

so that $c_{\rho} \in L_{0}$ if and only if $G_{K}^{n_{0} /\left(p^{2}-1\right)} \subset \operatorname{ker}(\rho)$. Moreover, since $m<p+1$ and $n_{0}>p^{2}-1$, we have $L_{0}=H_{\mathrm{gt}}^{1}\left(G_{K}, \overline{\mathbf{F}}_{p}(\chi)\right)=H_{\mathrm{cg}}^{1}\left(G_{K}, \overline{\mathbf{F}}_{p}(\chi)\right)$, so that these are precisely the gently ramified classes, which in this case coincide with the cogently ramified classes.

We remark that if $a_{0}=p-2$, we have $L_{V_{(p-2, p-1),(1,1)}} \subset H_{\mathrm{fl}}^{1}\left(G_{K}, \overline{\mathbf{F}}_{p}(\chi)\right)$ by [11]; together with the equality $L_{V_{(p-2, p-1)(1,1)}}=L_{V_{(p-2, p-1),(p, p)}}$ provided by [6], it follows in this particular case that $W^{\mathrm{AH}}(\rho)=W(\rho)$.

8.5. Two irreducible cases. For completeness, we also list the possibilities when $\rho$ is irreducible. Recall that in this case we let $W^{\mathrm{AH}}(\rho)=W(\rho)$ as defined in (4).

We let $K^{\prime}$ denote the unramified quadratic extension of $k$ and $k^{\prime}$ its residue field. Choose an embedding $\tau^{\prime}: k^{\prime} \rightarrow \overline{\mathbf{F}}_{p}$ and let $\psi=\omega_{\tau^{\prime}}: I_{K} \rightarrow \overline{\mathbf{F}}_{p}^{\times}$denote the associated fundamental character, so $\psi$ has order $p^{4}-1$. We then have

$$
\left.\rho\right|_{I_{K}} \sim\left(\begin{array}{cc}
\psi^{a} & 0 \\
0 & \psi^{p^{2} a}
\end{array}\right)
$$

for some $a$ with $1 \leqslant a \leqslant p^{4}-1$ and $a \neq \equiv 0 \bmod p^{2}+1$. Twisting by characters of $G_{K}$, we may alter $a$ by multiples of $p^{2}+1$ and hence assume $1 \leqslant a \leqslant p^{2}$. Altering our choice of $\tau^{\prime}$, we may further assume $a=a_{0}+a_{1} p$ where either

(IV) $2 \leqslant a_{0} \leqslant p-1$ and $1 \leqslant a_{1} \leqslant p-2$, or

(V) $1 \leqslant a_{0} \leqslant p-1$ and $a_{1}=0$.

In Case IV, which is equivalent to $a \not \equiv i p^{j} \bmod p^{2}+1$ for $i=1, \ldots, p-1$, $j=0,1,2,3$, we find that 


$$
\begin{aligned}
& W(\rho)=\left\{V_{(0,0),\left(a_{0}, a_{1}\right)}, V_{\left(a_{0}-1, a_{1}\right),\left(p+1-a_{0}, p-1-a_{1}\right)}, V_{\left(a_{0}-1, p-1\right),\left(p-a_{0}, a_{1}+1\right)},\right. \\
& \left.V_{\left(0, a_{1}\right),\left(a_{0}-1, p-a_{1}\right)}\right\}
\end{aligned}
$$

where the indices in $T$ are ordered so the first embedding is the restriction of our chosen $\tau^{\prime}$. In case $\mathrm{V}$, we find that

$$
\begin{gathered}
W(\rho)=\left\{V_{(p-2, p-1),\left(a_{0}+1, p\right)}, V_{\left(a_{0}-1,0\right),\left(p+1-a_{0}, p-1\right)}, V_{\left(a_{0}-1, p-1\right),\left(p-a_{0}, 1\right)},\right. \\
\left.V_{(0,0),\left(a_{0}-1, p\right)}\right\}
\end{gathered}
$$

with the last weight omitted if $a_{0}=1$.

\section{Examples of Galois representations}

We now illustrate the possible behaviour discussed in the preceding section with eight explicit examples for $p=3, f=2$. In the next section, we exhibit in Table 3 numerically matching automorphic data for each of the Galois representations described here. We refer to [9] for an extensive collection of examples for more general $p$ and $f$ and elaboration on methods for obtaining and analysing them.

We are restricting here to $p=3$, as this is the smallest prime for which all the reducible Cases I, II and III arise. We organize the examples according to the classification in the preceding section, and we content ourselves with examples for each type labelled $(a)$ or $\left(b_{i}\right)$ as these already illustrate the main new phenomena involving wild ramification in the quadratic case.

In the first two examples, $F=\mathbf{Q}(\sqrt{2})$ while in the last six, $F=\mathbf{Q}(\sqrt{5})$. All our representations $\rho$ take values in $\mathrm{GL}_{2}(k)$, where $k=\mathcal{O}_{F} / 3 \mathcal{O}_{F}$ is viewed as a subfield of $\overline{\mathbf{F}}_{3}$ via the embedding labelled $\tau_{0}$. We let $\alpha$ denote a root of $x^{2}+2 x-1$ if $F=\mathbf{Q}(\sqrt{2})$, and a root of $x^{2}-x-1$ if $F=\mathbf{Q}(\sqrt{5})$, and in either case we use the same symbol $\alpha$ for its image in $k \subset \overline{\mathbf{F}}_{3}$.

In the list below, we describe $\rho$ by specifying its projective splitting field, its conductor (prime to 3) and its local behaviour at $p=3$ up to an unramified quadratic twist. In each of our examples one can show there is a unique representation $\rho$ satisfying this description, except for those in Case III, where there are two such representations differing by a quadratic twist.

9.1. Case I. We use examples with tame signature $\left(a_{0}, a_{1}\right)=(1,2)$, so $n_{0}=7$ and $n_{1}=5$. This means that $\left.\rho\right|_{G_{K}}$ is a twist of a representation of the form

$$
\left(\begin{array}{ll}
\chi & * \\
0 & 1
\end{array}\right), \quad \text { with }\left.\chi\right|_{I_{K}}=\omega_{\tau_{0}}^{7}=\omega_{\tau_{1}}^{5}
$$

in particular $\chi$ is primitive and generic. 
In all our examples, $\chi$ will in fact have the form $\omega_{\pi}^{7}: \operatorname{Gal}(M / K) \rightarrow k^{\times}$where $\pi^{8}$ is a uniformizer of $K, M=K(\pi)$ and $\omega_{\pi}$ is the associated fundamental character. The class $c_{\rho} \in H^{1}\left(G_{K}, k\left(\omega_{\pi}^{7}\right)\right)$ thus corresponds via local class field theory to $\operatorname{aral}(M / K)$-linear homomorphism

$$
M^{\times} \longrightarrow \operatorname{Gal}(N / M) \cong k\left(\omega_{\pi}^{7}\right)
$$

with kernel $\operatorname{Norm}_{N / M}\left(N^{\times}\right)$where $N$ is the projective splitting field of $\left.\rho\right|_{G_{K}}$. This kernel contains $I M^{\times}$, where $I$ is the kernel of the surjection $\mathbf{Z}[\mathrm{Gal}(M / K)] \rightarrow k$ induced by $\omega_{\pi}^{7}$. As a $k$-vector space $M^{\times} / I M^{\times}$is two-dimensional, spanned by $E_{3}\left(\pi^{5}\right) \equiv 1+\pi^{5}$ and $E_{3}\left(\pi^{7}\right) \equiv 1+\pi^{7}$, and $\operatorname{Norm}_{N / M}\left(N^{\times}\right) / I M^{\times}$is a onedimensional subspace that determines $W^{\mathrm{AH}}\left(\left.\rho\right|_{G_{K}}\right)$ via (12).

Example Ia. Let $F=\mathbf{Q}(\sqrt{2})$ and let $E$ denote the splitting field over $\mathbf{Q}$ of the polynomial

$$
f_{\mathrm{Ia}}(x)=x^{10}-24 x^{7}-42 x^{6}-24 x^{4}-48 x^{3}-18 x^{2}-32 x-96 .
$$

Then $F \subset E$, and there is an isomorphism $\varrho: \operatorname{Gal}(E / F) \rightarrow \mathrm{PGL}_{2}(k)$ that lifts to a representation $\rho: G_{F} \rightarrow \mathrm{GL}_{2}(k)$ of conductor $\mathfrak{p}_{2}^{6}$, where $\mathfrak{p}_{2}=(\sqrt{2})$.

Up to an unramified quadratic twist, the local representation $\left.\rho\right|_{G_{K}}$ has the form

$$
\omega_{\pi}^{5} \otimes\left(\begin{array}{cc}
\omega_{\pi}^{7} & * \\
0 & 1
\end{array}\right)
$$

where $\pi^{8}=3$, and the splitting field $N$ of the projective local representation is that of the polynomial $x^{9}+6 x^{7}+3 x^{6}+6$. Here and in the later examples, we are using the database described in [16] to pass from the global polynomial to a local 3-adic Eisenstein polynomial. As will be explained in more detail in [9], the above form for the local representation is determined up to twist by the maximal tamely ramified subfield of $N$ (in this case $M=K(\pi)$ ), the action of $\operatorname{Gal}(M / K)$ on $\operatorname{Gal}(N / M)$ and the choice of isomorphism $\varrho$. The twist is then specified, up to an unramified quadratic character, as part of the data characterizing the lift $\rho$ of the projective representation $\varrho$. Using a Magma program described in [9], we find that $\operatorname{Norm}_{N / M}\left(N^{\times}\right) / I M^{\times}$consists of the classes of elements of the form $1+[a] \pi^{5}-[a]^{3} \pi^{7}$ for $a \in k$. Taking into account the twist by $\omega_{\pi}^{5}$, we conclude from (12) that $W^{\mathrm{AH}}\left(\left.\rho\right|_{G_{K}}\right)=\left\{V_{(2,1),(1,2)}\right\}$.

Example $\mathbf{I b}_{1}$. Let $F=\mathbf{Q}(\sqrt{2})$ again and let $E$ denote the splitting field over $F$ of the polynomial

$$
f_{\mathrm{Ib}_{1}}(x)=x^{10}-9 x^{8}+78 x^{6}-246 x^{4}-48 x^{3}+459 x^{2}+224 x-75 .
$$

We again have $F \subset E$ and an isomorphism $\operatorname{Gal}(E / F) \cong \mathrm{PGL}_{2}(k)$ lifting to a representation $\rho: G_{F} \rightarrow \mathrm{GL}_{2}(k)$ of conductor $\mathfrak{p}_{2}^{6}$. 
Up to an unramified quadratic twist, $\left.\rho\right|_{G_{K}}$ has the form

$$
\omega_{\pi}^{2} \otimes\left(\begin{array}{cc}
\omega_{\pi}^{7} * \\
0 & 1
\end{array}\right)
$$

where now $\pi^{8}=-3$ and the local projective splitting field $N$ is that of $x^{9}+3 x^{7}+3$. In this case however $\operatorname{Norm}_{N / M}\left(N^{\times}\right) / I M^{\times}$consists of the classes of $1+[a] \pi^{5}$ for $a \in k$, so (12) implies that $c_{\rho} \in L_{1}$. Taking into account the twist by $\omega_{\pi}^{2}$, we conclude that $W^{\mathrm{AH}}\left(\left.\rho\right|_{G_{K}}\right)=\left\{V_{(2,0),(1,2)}, V_{(0,0),(2,3)}\right\}$.

Example $\mathbf{I} \mathbf{b}_{2}$. Now, and for all the remaining examples, let $F=\mathbf{Q}(\sqrt{5})$. Let $E$ denote the splitting field over $\mathbf{Q}$ of the polynomial $f_{\mathrm{Ib}_{2}}(x)=$ $x^{10}-2 x^{9}+9 x^{8}+48 x^{7}-132 x^{6}+504 x^{5}+228 x^{4}-1824 x^{3}+6894 x^{2}-7676 x+4462$.

We again have $F \subset E$ and an isomorphism $\operatorname{Gal}(E / F) \cong \mathrm{PGL}_{2}(k)$ lifting to a representation $\rho: G_{F} \rightarrow \mathrm{GL}_{2}(k)$ of conductor $(2)^{5}$.

Up to an unramified quadratic twist, $\left.\rho\right|_{G_{K}}$ has the form

$$
\omega_{\pi}^{7} \otimes\left(\begin{array}{cc}
\omega_{\pi}^{7} & * \\
0 & 1
\end{array}\right)
$$

where $\pi^{8}=-3$ and the local projective splitting field $N$ is that of $x^{9}+6 x^{5}+6$. We now find that $\operatorname{Norm}_{N / M}\left(N^{\times}\right) / I M^{\times}$consists of the classes of $1+[a] \pi^{7}$ for $a \in k$, so that $c_{\rho} \in L_{0}$ by (12) (or by (11) since $G_{K}^{7 / 8} \subset \operatorname{ker}(\rho)$ ). Taking into account the twist by $\omega_{\pi}^{7}$, we conclude that $W^{\mathrm{AH}}\left(\left.\rho\right|_{G_{K}}\right)=\left\{V_{(1,2),(1,2)}, V_{(1,1),(2,1)}\right\}$.

9.2. Case II. We use examples with tame signature $\left(a_{0}, a_{1}\right)=(1,1)$, so $n_{0}=n_{1}=4$. Thus $\left.\rho\right|_{G_{K}}$ is a twist of a representation of the form

$$
\left(\begin{array}{ll}
\chi & * \\
0 & 1
\end{array}\right), \quad \text { with }\left.\chi\right|_{I_{K}}=\omega_{\tau_{0}}^{4}=\omega_{\tau_{1}}^{4} ;
$$

in particular $\chi$ is imprimitive and generic.

Note that we may write $\chi=\mu \omega_{\pi}$ where $\mu$ is unramified and $\pi^{2}=-3$, so $\omega_{\pi}$ is the cyclotomic character. In all our examples, we will have $c_{\rho} \in H_{\mathrm{ty}}^{1}\left(G_{K}, k(\chi)\right)$, and since $H_{\mathrm{ur}}^{1}\left(G_{K}, k(\chi)\right)=0$, we see that $W^{\mathrm{AH}}\left(\left.\rho\right|_{G_{K}}\right)$ is determined by whether $c_{\rho}$ is a multiple of either of the classes defined in (14).

Example IIa. Let $E$ be the splitting field over $\mathbf{Q}$ of the polynomial

$$
f_{\text {IIa }}(x)=x^{4}-x^{3}+2 x-11 .
$$


A representation $\rho: G_{F} \rightarrow \mathrm{GL}_{2}\left(\mathbf{F}_{3}\right)$ of conductor (7) with projective splitting field $E$ is given by the 3-torsion of a quadratic twist of the base change to $F=\mathbf{Q}(\sqrt{5})$ of the elliptic curve over $\mathbf{Q}$ with Cremona label 175A.

Up to an unramified quadratic twist, the local representation $\left.\rho\right|_{G_{K}}$ has the form

$$
\left(\begin{array}{rr}
\omega_{\pi} & * \\
0 & 1
\end{array}\right)
$$

where $\pi^{2}=-3$, so we can take $M=K(\pi)$ and $l=k$. The local projective splitting field $N$ is that of $x^{3}+3 x+3$. Since $\omega_{\pi}$ is cyclotomic, we have that $H_{\mathrm{fl}}^{1}\left(G_{K}, \mathbf{F}_{3}\left(\omega_{\pi}\right)\right)=H_{\mathrm{ty}}^{1}\left(G_{K}, \mathbf{F}_{3}\left(\omega_{\pi}\right)\right)$ has codimension one in $H^{1}\left(G_{K}, \mathbf{F}_{3}\left(\omega_{\pi}\right)\right)$. One can check directly that $G_{K}^{3 / 2} \subset \operatorname{ker}(\rho)$ and hence that $c_{\rho} \in H_{\mathrm{ty}}^{1}\left(G_{K}, \mathbf{F}_{3}\left(\omega_{\pi}\right)\right)$, or deduce this from the fact $\rho$ is defined by an elliptic curve with good ordinary reduction at 3. On the other hand since $c_{\rho}$ is nontrivial and takes values in $\mathbf{F}_{3}$, but the homomorphism in (14) is simply $\tau_{i}$ and hence has image of order 9, it follows that $c_{\rho} \notin L_{0} \cup L_{1}$. Therefore, $W^{\mathrm{AH}}\left(\left.\rho\right|_{G_{K}}\right)=\left\{V_{(0,0),(3,3)}, V_{(0,0),(1,1)}\right\}$.

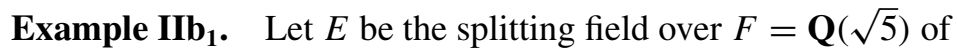
$f_{\mathrm{IIb}_{1}}(x)=x^{6}-3 \alpha x^{5}+3 \alpha x^{4}+(6 \alpha+6) x^{3}-(21 \alpha+12) x^{2}+(21 \alpha+12) x-8 \alpha-4$.

The Shimura curve associated to the units of a maximal order of a quaternion algebra over $F$ ramified at one archimedean place and the prime $\mathfrak{p}_{61}=(3-7 \alpha)$ has genus two, and its Jacobian has real multiplication by $F$ (see [8, Remark 3]). The 3-torsion points of this Jacobian give rise to a representation $\rho: G_{F} \rightarrow \mathrm{GL}_{2}(k)$ of conductor $\mathfrak{p}_{61}$ with $E$ as its projective splitting field. Note that unlike the preceding examples, $E$ is not Galois over $\mathbf{Q}$.

Up to an unramified quadratic twist, the local representation $\left.\rho\right|_{G_{K}}$ has the form

$$
v^{-1} \otimes\left(\begin{array}{cc}
v^{2} \omega_{\pi} & * \\
0 & 1
\end{array}\right)
$$

where $\pi^{2}=-3$ and $v$ is the unramified character of $G_{K}$ sending Frob Fo $_{K}$ to reduction of) $\alpha^{3}$. We let $M=L(\pi)$ where $L$ is the unramified extension of $K$ of degree 4 , so also $[l: k]=4$. The splitting field of the character $v^{2} \omega_{\pi}$ is not of the form required for the construction of Section 5, so we have adjoined $\pi$ in order to obtain a field of the required form; note that the extension $M / K$ is not cyclic. Note also that since $v^{2}$ is nontrivial, we have $H_{\mathrm{ty}}^{1}\left(G_{K}, k\left(v^{2} \omega_{\pi}\right)\right)=H^{1}\left(G_{K}, k\left(v^{2} \omega_{\pi}\right)\right)$. The class $c_{\rho}$ now corresponds to a $\operatorname{Gal}(M / K)$-linear homomorphism

$$
M^{\times} / I M^{\times} \longrightarrow \operatorname{Gal}(N / M) \cong k\left(v^{2} \omega_{\pi}\right)
$$


where $I$ is the kernel of the surjection $\mathbf{Z}[\operatorname{Gal}(M / K)] \rightarrow k$ induced by $v^{2} \omega_{\pi}$, and $N$ is the composite of $M$ with the projective local splitting field of $\rho$. As a $k$ vector space, $M^{\times} / I M^{\times}$is two-dimensional, consisting of the classes of $E_{3}([a]) \equiv$ $1+[a] \pi$ for $a$ in the kernel of $\operatorname{tr}_{l / k^{\prime}}$, where $k^{\prime}$ is the quadratic extension of $k$. Unravelling (14), we find that $c_{\rho} \in L_{0}$ (respectively $L_{1}$ ) if and only if $c_{\rho}$ is trivial on those $1+[a] \pi$ such that $a^{8}=v^{2}\left(\right.$ Frob $\left._{K}\right)=\alpha^{2}\left(\right.$ respectively $a^{8}=v^{6}\left(\right.$ Frob $\left._{K}\right)=$ $\left.-\alpha^{2}\right)$. Explicit computation of elements of $\operatorname{Norm}_{N / M}\left(N^{\times}\right)$shows that indeed $c_{\rho}$ is in $L_{1}$ (and hence not in $L_{0}$ since $c_{\rho} \neq 0$ ), so that $W^{\mathrm{AH}}\left(\left.\rho\right|_{G_{K}}\right)=\left\{V_{(0,0),(3,3)}\right.$, $\left.V_{(0,0),(1,1)}, V_{(0,2),(2,2)}\right\}$.

Example II I $_{2}$. We may take Example IIb I $_{1}$ and replace $\rho$ by $\rho \circ \sigma$, where $\sigma$ is the outer automorphism of $G_{F}$ induced by conjugation by an element of $G_{\mathbf{Q}}$ extending the nontrivial element of $\operatorname{Gal}(F / \mathbf{Q})$. The resulting representation has conductor $\mathfrak{p}_{61}^{\prime}=(4-7 \alpha)$ and projective splitting field $\sigma(E)$; the character $v$ in the description of $\left.\rho\right|_{G_{K}}$ is the same as in Example $\mathrm{IIb}_{1}$, but the kernel of the homomorphism induced by $c_{\rho}$ would be replaced by its Galois conjugate. We therefore conclude that $c_{\rho}$ is in $L_{0}$ instead of $L_{1}$, so that $W_{\mathrm{AH}}\left(\left.\rho\right|_{G_{K}}\right)=\left\{V_{(0,0),(3,3)}\right.$, $\left.V_{(0,0),(1,1)}, V_{(2,0),(2,2)}\right\}$. The corresponding system of Hecke eigenvalues is obtained from the one in Example $\mathrm{IIb}_{1}$ by interchanging each $a_{v}$ with $a_{\sigma(v)}$. (Note that a similar procedure could not have been used to generate an example of type $\mathrm{Ib}_{2}$ from $\mathrm{Ib}_{1}$ since the inequality $n_{1}<n_{0}$ would not be preserved.)

Alternatively, we could obtain an example of type $\mathrm{IIb}_{2}$ by replacing the representation $\rho$ in Example $\mathrm{IIb}_{1}$ by its composite with the automorphism of $\mathrm{GL}_{2}(k)$ induced by Frob on $k$. The projective splitting field is then the same as in Example $\mathrm{IIb}_{1}$, as is the description of $\left.\rho\right|_{G_{K}}$, except that $v$ is replaced by the unramified character sending Frob $_{K}$ to $\alpha$ and the homomorphism corresponding to $c_{\rho}: M^{\times} \rightarrow k\left(v^{2} \omega_{\pi}\right)$ is obtained from the preceding one by composing with Frob. Note that $N$ and $I M^{\times}$do not change, but the criteria for $c_{\rho}$ to be in $L_{0}$ and $L_{1}$ in terms of $\operatorname{Norm}_{N / M}\left(N^{\times}\right)$are interchanged. In this case the corresponding system of Hecke eigenvalues is obtained from the one in Example $\mathrm{IIb}_{1}$ by replacing each $a_{v}$ with $\operatorname{Frob}\left(a_{v}\right)$. Finally of course, we could just as well have obtained an example of type $\mathrm{IIb}_{1}$ by replacing the original $\rho$ with Frob $\circ \rho \circ \sigma$.

9.3. Case III. We use examples with tame signature $\left(a_{0}, a_{1}\right)=(1,3)$, so $n_{0}=10$ and $n_{1}=6$. Thus $\left.\rho\right|_{G_{K}}$ is a twist of a representation of the form

$$
\left(\begin{array}{ll}
\chi & * \\
0 & 1
\end{array}\right), \quad \text { with }\left.\chi\right|_{I_{K}}=\omega_{\tau_{0}}^{2}=\omega_{\tau_{1}}^{6}
$$

in particular $\chi$ is primitive and nongeneric. 
In both our examples $\chi$ will in fact have the form $\omega_{\pi}: \operatorname{Gal}(M / K) \rightarrow k^{\times}$ where $\pi^{4}$ is a uniformizer of $K, M=K(\pi)$ and $\omega_{\pi}$ is the associated fundamental character. The class $c_{\rho}$ will be nontrivial, so that $W^{\mathrm{AH}}\left(\left.\rho\right|_{G_{K}}\right)$ is determined by whether $c_{\rho}$ lies in the space $L_{0}$ described in (15) or (16). Note also that since $a_{0}=p-2$, we know in fact in this case that $W^{\mathrm{AH}}\left(\left.\rho\right|_{G_{K}}\right)=W\left(\left.\rho\right|_{G_{K}}\right)$ by the remark at the end of Section 8.

For both examples, there are in fact two representations with the given description; choosing either to be $\rho$, the other is $\delta \otimes \rho$ where $\delta$ the nontrivial character of $\operatorname{Gal}\left(F\left(\zeta_{5}\right) / F\right)$.

Example IIIa. Let $E$ denote the splitting field over $\mathbf{Q}$ of the polynomial

$$
f_{\text {IIIa }}(x)=x^{10}-5 x^{9}+135 x^{6}-360 x^{5}+405 x^{4}-270 x^{3}+135 x^{2}-45 x+9 .
$$

We then have $F=\mathbf{Q}(\sqrt{5}) \subset E$ and an isomorphism $\operatorname{Gal}(E / F) \cong \mathrm{PSL}_{2}(k)$ lifting to a representation $\rho: G_{F} \rightarrow \mathrm{GL}_{2}(k)$ of conductor $\mathfrak{p}_{5}^{3}$, where $\mathfrak{p}_{5}=(\sqrt{5})$.

Up to an unramified quadratic twist, $\left.\rho\right|_{G_{K}}$ has the form

$$
\omega_{\varpi} \otimes\left(\begin{array}{cc}
\omega_{\varpi}^{2} & * \\
0 & 1
\end{array}\right)
$$

where $\varpi^{8}=3 \omega^{2}$, so we may take $M=K(\pi)$ with $\pi=\varpi^{2}$. The splitting field $N$ of the projective local representation is that of the polynomial $x^{9}+9 x+6$, and we find that $G_{K}^{5 / 4} \not \subset \operatorname{ker}(\rho)$, so that $c_{\rho} \notin L_{0}=\operatorname{Fil}^{5 / 4} H^{1}\left(G_{K}, \overline{\mathbf{F}}_{3}\left(\omega_{\pi}\right)\right)$ by (16). Taking into account the twist by $\omega_{\varpi}$, we conclude that $W^{\mathrm{AH}}\left(\left.\rho\right|_{G_{K}}\right)=\left\{V_{(1,0),(1,3)}\right\}$.

Example IIIb . Let $E$ denote the splitting field over $\mathbf{Q}$ of the polynomial

$$
f_{\mathrm{IIIb}_{1}}(x)=x^{6}-3 x^{5}+5 x^{3}-5 .
$$

We again have $F \subset E$ and an isomorphism $\operatorname{Gal}(E / F) \cong \mathrm{PSL}_{2}(k)$ lifting to a representation $\rho: G_{F} \rightarrow \mathrm{GL}_{2}(k)$, now of conductor (2) $\mathfrak{p}_{5}^{3}$.

Up to an unramified quadratic twist, $\left.\rho\right|_{G_{K}}$ again has the form

$$
\omega_{\varpi} \otimes\left(\begin{array}{cc}
\omega_{\varpi}^{2} & * \\
0 & 1
\end{array}\right)
$$

but now $\varpi^{8}=3$, and we take $M=K(\pi)$ with $\pi=\varpi^{2}$. In contrast to the preceding example, we find that $G_{K}^{5 / 4} \subset \operatorname{ker}(\rho)$, so that $c_{\rho} \in L_{0}$ by (16). Taking into account the twist by $\omega_{\varpi}$, we conclude that $W^{\mathrm{AH}}\left(\left.\rho\right|_{G_{K}}\right)=\left\{V_{(1,0),(1,3)}\right.$, $\left.V_{(0,0),(3,3)}, V_{(0,0),(1,1)}\right\}$. 


\section{Numerical matching with automorphic forms}

To facilitate computations, both here and in the sequel [9], we work with algebraic automorphic forms on definite quaternion algebras over totally real fields. Recall that these are related to Hilbert modular forms by the JacquetLanglands correspondence, and under mild hypotheses give the set of weights of forms giving rise to $\rho$ in the sense of Conjectures 2.1 and 7.3.

More precisely, we consider totally real fields $F$ in which $p$ is inert and definite quaternion algebras $B$ over $F$ which are split at $p$, and we present pairs $(\phi, \rho)$ where:

- $\phi=\left(a_{v}, d_{v}\right)_{v \in \Sigma_{\phi}}$ is a system of eigenvalues for the standard Hecke operators $T_{v}$ and $S_{v}$ (as defined in [25]) acting on mod $p$ algebraic modular forms for $B$ of some level $\mathfrak{n}_{\phi}$ (where $\Sigma_{\phi}$ is a large set of good primes);

- $\rho: G_{F} \rightarrow \mathrm{GL}_{2}\left(\overline{\mathbf{F}}_{p}\right)$ is a Galois representation unramified outside $p \mathfrak{n}_{\phi}$ such that $\rho\left(\mathrm{Frob}_{v}\right)$ has characteristic polynomial $x^{2} \pm a_{v} x+d_{v} \mathbf{N}(v)$ for all $v \in \Sigma_{\phi}$;

- the set of weights for which $\phi$ occurs at level $\mathfrak{n}_{\phi}$ is precisely $W^{\mathrm{AH}}\left(\left.\rho\right|_{G_{F_{p}}}\right)$.

The reason for the sign ambiguity in the trace of $\rho\left(\mathrm{Frob}_{v}\right)$ is that in practice we work with the associated projective representation. The $\rho$ we consider, particularly in [9], are typically constructed independently from automorphic forms. The existence of a numerically matching $\phi$ can be viewed as evidence for the modularity part of Conjecture 7.3 (and hence Conjecture 2.1).

For each of the eight Galois representations $\rho$ from the previous section, we exhibit a corresponding $\phi$ here, taking $\Sigma_{\phi}$ to be all good primes with norm at most 100. The methods for computing $\phi$ are based on those described in [7] and [3, Appendix B].

10.1. A summarizing table. Table 1 summarizes our eight examples, adding some more information. Note that in all cases besides the conjugate cases $\mathrm{IIb}_{1}$ and $\mathrm{IIb}_{2}$, the polynomial $F_{c}(x):=f_{c}(x)$ has coefficients in $\mathbf{Q}$. Its Galois group is given in the $G$ column. In this column, an exceptional isomorphism identifies the group $\mathrm{PSL}_{2}(9)$ with the alternating group $A_{6}$. The group $\mathrm{P} \Gamma \mathrm{L}_{2}(9)=\operatorname{Aut}\left(\mathrm{PSL}_{2}(9)\right)$ contains $\mathrm{PSL}_{2}(9) \cong A_{6}$ with index four and the three intermediate groups are $M_{10}, \mathrm{PGL}_{2}(9)$, and $S_{6}$. The entry shared by the $\mathrm{IIb}_{1}$ and $\mathrm{IIb}_{2}$ rows is the Galois group of the product $F_{\mathrm{IIb}}(x):=f_{\mathrm{IIb}_{1}}(x) f_{\mathrm{IIb}_{2}}(x) \in \mathbf{Z}[x]$. The $D$ column gives the field discriminant of $\mathbf{Q}[x] / F_{c}(x)$. The largest slope $s$ is explained in the next subsection. 
Table 1. Information on the eight examples. The weight in ordinary type is computed from the tame signature. As explained in Section 10.2, weights in italics come from small slopes and weights in boldface come from other sources.

\begin{tabular}{|c|c|c|c|c|c|c|c|c|}
\hline Ex. & $F$ & $G$ & $D$ & $s$ & $\mathfrak{n}$ & $\mathbf{N}(\mathfrak{n})$ & $\mathfrak{f}$ & Weights $\left(W^{\mathrm{AH}}(\rho)\right)$ \\
\hline Ia & $\mathbf{Q}(\sqrt{2})$ & $\mathrm{P} \Gamma \mathrm{L}_{2}(9)$ & $2^{27} 3^{15}$ & $15 / 8$ & $\mathfrak{p}_{2}^{6}$ & 64 & $\mathfrak{p}_{2}^{6}$ & {$[2,1 ; 1,2]$} \\
\hline $\mathrm{Ib}_{1}$ & $\mathbf{Q}(\sqrt{2})$ & $\mathrm{P} \Gamma \mathrm{L}_{2}(9)$ & $2^{28} 3^{15}$ & $15 / 8$ & $\mathfrak{p}_{2}^{6}$ & 64 & $\mathfrak{p}_{2}^{6}$ & {$[2,0 ; 1,2],[\mathbf{0}, \mathbf{0} ; \mathbf{2}, \mathbf{3}]$} \\
\hline $\mathrm{Ib}_{2}$ & $\mathbf{Q}(\sqrt{5})$ & $\mathrm{P} \Gamma \mathrm{L}_{2}(9)$ & $2^{31} 3^{13} 5^{3}$ & $13 / 8$ & $(2)^{5}$ & 1024 & $(2)^{5}$ & {$[1,2 ; 1,2],[1,1 ; 2,1]$} \\
\hline IIa & $\mathbf{Q}(\sqrt{5})$ & $S_{4}$ & $-3^{3} 5^{2} 7^{2}$ & $3 / 2$ & (7) & 49 & $\mathcal{O}$ & {$[0,0 ; 3,3],[0,0 ; 1,1]$} \\
\hline $\mathrm{IIb}_{1}$ & $\mathbf{Q}(\sqrt{5})$ & & & & $\mathfrak{p}_{61}$ & 61 & $\mathcal{O}$ & {$[0,0 ; 3,3],[0,0 ; 1,1],[\mathbf{0}, \mathbf{2} ; \mathbf{2}, \mathbf{2}]$} \\
\hline $\mathrm{IIb}_{2}$ & $\mathbf{Q}(\sqrt{5})$ & $A_{6}^{2} \cdot 2$ & & $3 / 2$ & $\mathfrak{p}_{61}^{\prime}$ & 61 & $\mathcal{O}$ & {$[0,0 ; 3,3],[0,0 ; 1,1],[\mathbf{2}, \mathbf{0} ; \mathbf{2}, \mathbf{2}]$} \\
\hline IIIa & $Q(\sqrt{5})$ & $M_{10}$ & $3^{18} 5^{10}$ & $9 / 4$ & $\mathfrak{p}_{5}^{3}$ & 125 & $\mathcal{O}$ & {$[1,0 ; 1,3]$} \\
\hline $\mathrm{IIIb}_{1}$ & $\mathbf{Q}(\sqrt{5})$ & $S_{6}$ & $2^{2} 3^{6} 5^{2}$ & $5 / 4$ & (2) $\mathfrak{p}_{5}^{3}$ & 500 & $\mathcal{O}$ & {$[1,0 ; 1,3],[0,0 ; 3,3],[0,0 ; 1,1]$} \\
\hline
\end{tabular}

10.2. Slopes. For a separable polynomial $f(x) \in \mathbf{Q}_{p}[x]$, wild ramification in the algebra $A=\mathbf{Q}_{p}[x] / f(x)$ can be measured by slopes, as explained in [16]. These slopes are breaks in the upper numbering of [23, IV.3], increased by 1 . When all factors of $f(x)$ have degree $\leqslant 11$, they are computed automatically by the website of [16].

A common situation in our current setting is that $f(x) \in \mathbf{Q}[x]$ has degree ten, and factors over $\mathbf{Q}_{3}$ into a primitive nonic and a linear factor, giving $A=B \times \mathbf{Q}_{3}$. In this case, the primitive nonic field $B$ has a certain largest slope $s$ with multiplicity eight and 0 with multiplicity one. As $\mathbf{Q}_{3}$ has the trivial slope 0 as well, $\operatorname{ord}_{3}(D)=8 \mathrm{~s}$. This situation occurs in our four cases with $F_{c}(x)$ decic, namely $\mathrm{Ia}, \mathrm{Ib}_{1}, \mathrm{Ib}_{2}$, and IIIa. The other cases are similar. For example, $\mathbf{Q}_{3}[x] / f_{\text {IIIb }}(x)$ is a sextic field with a tame subfield of degree two. In this case, $\operatorname{ord}_{3}(D)=6$ decomposes as $4 s+1+0$; the 1 comes from the tame subfield and $s=5 / 4$ is the quantity of current interest.

The slope column illustrates that some extra weights come simply from $s$ being smaller than the maximum allowed by the tame signature. For example, for the tame signature $\left(a_{0}, a_{1}\right)=(2,2)$, the maximum allowed $s$ is $5 / 2$, while our examples are peu ramifiée and have slope $3 / 2$. However, other extra weights are not simple consequences of small slopes. The sequel paper [9] will illustrate a principle clear from the theory here: as the local degree $\left[K: \mathbf{Q}_{p}\right]$ increases, slopes account for a decreasing fraction of the phenomenon of extra weights.

10.3. The class set $\mathbf{P G L}_{2}(9)^{\natural}$. Table 2 summarizes how one does projective matching for general $\rho$ into $\mathrm{GL}_{2}(k)$ where $k$ has order 9. On the automorphic 
Table 2. The class set $\mathrm{PGL}_{2}(9)^{\natural}$ and its view from the automorphic and Galois sides. The outer involution - of $\mathrm{PGL}_{2}(9)$ makes the interchanges $5 A \leftrightarrow 5 B, 8 A \leftrightarrow 8 B$, and $10 A \leftrightarrow 10 B$, and fixes the other five classes.

\begin{tabular}{|c|c|c|c|c|c|c|c|c|c|c|c|}
\hline$d_{v}$ & \multicolumn{6}{|c|}{$d_{v}$ is a square } & \multicolumn{5}{|c|}{$d_{v}$ is not a square } \\
\hline$b_{v}$ & 1 & 0 & 1 & 2 & $\alpha^{2}$ & $\alpha^{6}$ & 0 & $\alpha$ & $\alpha^{3}$ & $\alpha^{5}$ & $\alpha^{7}$ \\
\hline $\mathrm{PGL}_{2}(9)^{\natural}$ & 1 & $2 u$ & 3 & 4 & $5 A$ & $5 B$ & $2 v$ & $8 A$ & $8 B$ & $10 B$ & $10 A$ \\
\hline $\mathrm{PGL}_{2}(9)$ & $1^{10}$ & $2^{4} 1^{2}$ & $3^{3} 1$ & $4^{2} 1^{2}$ & $5^{2}$ & $5^{2}$ & $2^{5}$ & $81^{2}$ & $81^{2}$ & 10 & 10 \\
\hline$A_{6}$ & $1^{6}$ & $2^{2} 1^{2}$ & $3^{2}$ or $31^{3}$ & 42 & 51 & 51 & & & & & \\
\hline$S_{4}$ & $1^{4}$ & $2^{2}$ or $21^{2}$ & $2 \quad 31$ & 4 & & & & & & & \\
\hline
\end{tabular}

side, one has the pairs $\left(a_{v}, d_{v}\right) \in k \times k^{\times}$. On the Galois side, the most immediately available quantities are partitions $\lambda_{v}$ with parts being the degrees of the irreducible factors of $f_{c}(x)$ in the completed ring $\mathcal{O}_{v}$.

When $f_{c}(x)$ is chosen to be a decic in the standard way, the table explains how the projective quantities $b_{v}=a_{v}^{2} / d_{v} \mathbf{N}(v)$ correlate with the decic partitions $\lambda_{v}$. In fact, let $\mathrm{PGL}_{2}(9)^{\natural}$ be the set of conjugacy classes in the group $\mathrm{PGL}_{2}(9)$. Then the Frobenius class $\mathrm{Fr}_{v} \in \mathrm{PGL}_{2}(9)^{\natural}$ determines both $b_{v}$ and $\lambda_{v}$. Conversely, the pair $\left(b_{v}, \lambda_{v}\right)$ determines $\mathrm{Fr}_{v}$. Using the FrobeniusElement command [10] in $M a g m a$, with adaptations to account for ground field $F$ rather than $\mathbf{Q}$, we have gone beyond partitions and have in all cases identified the correct label $A$ or $B$, directly from the polynomial $f_{c}(x)$. Table 2 also has lines corresponding to our sometimes replacing decic polynomials by sextic and quartic polynomials.

10.4. Matching for our eight examples. Table 3 is headed by the ten smallest split primes $p$ for each of the two fields $F$ in question. For each $p$, it gives one of the two $v$ above it. The conjugate prime $\sigma(v)$ is obtained by the substitution $\alpha \mapsto$ $-\alpha-2$ in the case $F=\mathbf{Q}(\sqrt{2})$ and $\alpha \mapsto 1-\alpha$ in the case $F=\mathbf{Q}(\sqrt{5})$. For each example, we list the classes $\mathrm{Fr}_{v} \in \mathrm{PGL}_{2}(9)^{\natural}$ associated to $\rho$ and the eigenvalues $a_{v}$ and $d_{v}$ of a numerically matching eigenform $\phi$. We omit the lines for $d_{v}$ when they are identically 1 . Recall that in Examples IIIa and $\mathrm{IIIb}_{1}$ there are two choices for $\rho$ differing by twist by the quadratic character $\delta: \operatorname{Gal}\left(\mathbf{Q}\left(\zeta_{5}\right) / F\right) \rightarrow\{ \pm 1\}$; accordingly we list the two matching eigenforms, each obtained from the other by replacing $a_{v}$ with $\delta(v) a_{v}$ where $\delta(v)=\delta\left(\operatorname{Fr}_{v}\right)=\left(\mathbf{N}_{F / \mathbf{Q}}(v) / 5\right)$.

For each $v$ listed in the table, the eigenvalues $a_{\sigma(v)}$ and $d_{\sigma(v)}$ can be recovered as follows: In all the examples, one has $d_{\sigma(v)}=d_{v}^{3}$, and in all but $\mathrm{IIb}_{1}, \mathrm{IIb}_{2}$ and $\mathrm{IIIb}_{1}$, one has $a_{\sigma(v)}=a_{v}^{3}$. In examples $\mathrm{IIb}_{1}$ and $\mathrm{IIb}_{2}$, with eigenvalues $a_{v}^{\prime}$ and $a_{v}^{\prime \prime}$ respectively, one has $a_{\sigma(v)}^{\prime}=a_{v}^{\prime \prime}$; finally in $\mathrm{IIIb}_{1}$, one has $a_{\sigma(v)}=\delta(v) a_{v}^{3}$. Similarly, 
Table 3. Matching in our eight examples for ten $v$.

$F=\mathbf{Q}(\sqrt{2})$

\begin{tabular}{l|llllllllll}
$p$ & 7 & 17 & 23 & 31 & 41 & 47 & 71 & 73 & 79 & 89
\end{tabular}

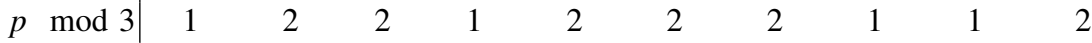

$v \mid 1+2 \alpha 2+3 \alpha 4-\alpha 3+4 \alpha 5-2 \alpha 6-\alpha 7+6 \alpha 7-2 \alpha 8-\alpha 10+7 \alpha$

\begin{tabular}{cc|cccccccccc} 
& $a_{v}$ & $\alpha^{3}$ & $\alpha^{3}$ & 0 & $\alpha^{3}$ & $\alpha$ & $\alpha^{2}$ & 2 & $\alpha^{3}$ & 1 & $\alpha^{2}$ \\
Ia & $d_{v}$ & $\alpha^{7}$ & $\alpha^{7}$ & $\alpha^{6}$ & $\alpha^{3}$ & $\alpha^{7}$ & $\alpha^{6}$ & $\alpha^{2}$ & 1 & $\alpha^{3}$ & $\alpha$
\end{tabular} $\begin{array}{lllllllllll}\mathrm{Fr}_{v} & 10 A & 8 B & 2 u & 8 B & 10 A & 5 A & 5 A & 5 B a & 10 B & 10 A\end{array}$

\begin{tabular}{cc|cccccccccc} 
& $a_{v}$ & 1 & $\alpha$ & $\alpha^{2}$ & $\alpha^{6}$ & $\alpha$ & $\alpha^{3}$ & 0 & $\alpha^{2}$ & $\alpha^{7}$ & 2 \\
$\mathrm{Ib}_{1}$ & $d_{v}$ & $\alpha$ & $\alpha^{5}$ & $\alpha^{6}$ & $\alpha$ & $\alpha$ & $\alpha^{2}$ & $\alpha^{2}$ & 2 & $\alpha$ & $\alpha^{7}$
\end{tabular}

$\begin{array}{lllllllllll}\mathrm{Fr}_{v} & 10 A & 8 A & 5 A & 8 B & 10 B & 3 & 2 u & 3 & 10 B & 10 B\end{array}$

$F=\mathbf{Q}(\sqrt{5})$

\begin{tabular}{l|llllllllll}
$p$ & 11 & 19 & 29 & 31 & 41 & 59 & 61 & 71 & 79 & 89
\end{tabular}

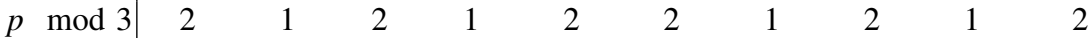

$v 2-3 \alpha 1-4 \alpha 5+\alpha 3-5 \alpha 6+\alpha 2-7 \alpha 4-7 \alpha 8+\alpha 5-8 \alpha 10-\alpha$

\begin{tabular}{cc|cccccccccc} 
& $a_{v}$ & $\alpha^{2}$ & $\alpha^{6}$ & $\alpha$ & $\alpha^{7}$ & $\alpha$ & 2 & $\alpha$ & $\alpha$ & $\alpha^{7}$ & $\alpha^{2}$ \\
$\mathrm{Ib}_{2}$ & $d_{v}$ & $\alpha^{3}$ & $\alpha^{2}$ & 2 & $\alpha$ & $\alpha^{5}$ & $\alpha^{3}$ & $\alpha^{3}$ & 2 & $\alpha$ & $\alpha^{7}$
\end{tabular}

$\begin{array}{lllllllllll}\mathrm{Fr}_{v} & 10 B & 5 A & 5 A & 10 B & 8 A & 8 A & 10 A & 5 A & 10 B & 8 A\end{array}$

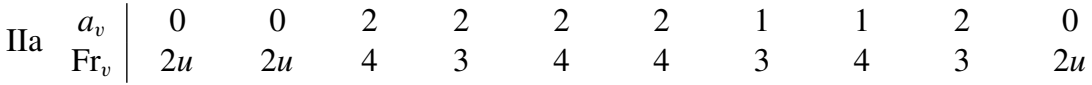

\begin{tabular}{cc|cccccccccc}
$\mathrm{IIb}_{1}$ & $a_{v}$ & $\alpha^{7}$ & 0 & $\alpha^{6}$ & $\alpha^{5}$ & $\alpha$ & $\alpha^{3}$ & 0 & 2 & $\alpha^{3}$ & 1 \\
& $\mathrm{Fr}_{v}$ & $5 A$ & $2 u$ & 3 & $5 A$ & $5 B$ & $5 A$ & $2 u$ & 4 & $5 B$ & 4
\end{tabular}

\begin{tabular}{lc|ccccccccc}
$\mathrm{IIb}_{2}$ & $a_{v}$ & $\alpha^{6}$ & $\alpha^{6}$ & $\alpha^{3}$ & $\alpha^{2}$ & $\alpha^{7}$ & $\alpha$ & $\alpha^{5}$ & $\alpha^{6}$ & $\alpha^{6}$ \\
& $\mathrm{Fr}_{v}$ & 3 & 4 & $5 A$ & 4 & $5 A$ & $5 B$ & $5 B$ & 4 & 3
\end{tabular}

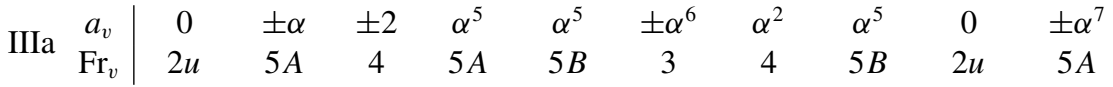

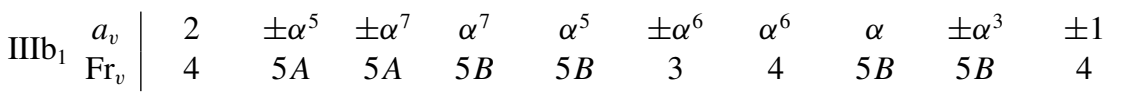


in all the examples but $\mathrm{IIb}_{1}$ and $\mathrm{IIb}_{2}$, one has $\mathrm{Fr}_{\sigma(v)}=\overline{\mathrm{Fr}}_{v}$. In examples $\mathrm{IIb}_{1}$ and $\mathrm{IIb}_{2}$, with Frobenius classes $\operatorname{Fr}_{v}^{\prime}$ and $\operatorname{Fr}_{v}^{\prime \prime}$ respectively, one has $\operatorname{Fr}_{\sigma(v)}^{\prime}=\operatorname{Fr}_{v}^{\prime \prime}$. Thus, for example, the first split prime $v=(1+2 \alpha)$ for $\mathbf{Q}(\sqrt{2})$ has conjugate $\sigma(v)=(3+2 \alpha)$, and in example Ia, one has $a_{\sigma(v)}=\alpha, d_{\sigma(v)}=\alpha^{5}$ and $\mathrm{Fr}_{\sigma(v)}=10 B$.

The agreement exhibited on Table 3 extends also to those $v$ with $\mathbf{N}(v)<100$ which do not have a place on the table.

\section{Acknowledgements}

We are grateful to Laurent Berger for the suggestion of considering the ArtinHasse exponential in the context of a related question, and to David Savitt and Michael Schein for discussions that confirmed the compatibility of Conjecture 7.2 with the results of [15]. We thank Victor Abrashkin for calling our attention to the paper [1], and Toby Gee for informing us of work on Conjecture 7.2 leading to its proof in [5]. We would also like to thank the referee for numerous suggestions that improved the exposition of this paper.

This research was partially supported by EPSRC Grant EP/J002658/1 (LD), Leverhulme Trust RPG-2012-530, EPSRC Grant EP/L025302/1 and the Heilbronn Institute for Mathematical Research (FD), and Simons Foundation Collaboration Grant \#209472 (DPR).

\section{References}

[1] V. Abrashkin, 'Modular representations of the Galois group of a local field and a generalization of a conjecture of Shafarevich', Izv. Akad. Nauk SSSR Ser. Mat. 53(6) (1989), 1135-1182. 1337; MR 1039960.

[2] A. Ash and W. Sinnott, 'An analogue of Serre's conjecture for Galois representations and Hecke eigenclasses in the mod $p$ cohomology of GL(n, Z)', Duke Math. J. 105(1) (2000), 1-24; MR 1788040.

[3] C. Breuil, 'Sur un problème de compatibilité local-global modulo $p$ pour $\mathrm{GL}_{2}$ ', J. Reine Angew. Math. 692 (2014), 1-76; MR 3274546.

[4] K. Buzzard, F. Diamond and F. Jarvis, 'On Serre's conjecture for mod $\ell$ Galois representations over totally real fields', Duke Math. J. 155(1) (2010), 105-161; MR 2730374.

[5] F. Calegari, M. Emerton, T. Gee and L. Mavrides, 'Explicit Serre weights for two-dimensional Galois representations', Preprint, 2016, arXiv:1608.06059.

[6] S. Chang and F. Diamond, 'Extensions of rank one $(\phi, \Gamma)$-modules and crystalline representations', Compositio Math. 147(2) (2011), 375-427; MR 2776609.

[7] L. Dembélé , 'Explicit computations of Hilbert modular forms on $\mathbb{Q}(\sqrt{5})$ ', Exp. Math. 14(4) (2005), 457-466; MR 2193808.

[8] L. Dembélé, 'Quaternionic Manin symbols, Brandt matrices, and Hilbert modular forms', Math. Comp. 76(258) (2007), 1039-1057; MR 2291849. 
[9] L. Dembélé, F. Diamond and D. P. Roberts, 'On the computation and combinatorics of Serre weights for two-dimensional Galois representations', in preparation.

[10] T. Dokchitser and V. Dokchitser, 'Identifying Frobenius elements in Galois groups', Algebra Number Theory 7(6) (2013), 1325-1352; MR 3107565.

[11] J.-M. Fontaine, 'Il n'y a pas de variété abélienne sur Z', Invent. Math. 81(3) (1985), 515-538; MR 807070.

[12] T. Gee, 'Automorphic lifts of prescribed types', Math. Ann. 350(1) (2011), 107-144; MR 2785764.

[13] T. Gee, F. Herzig and D. Savitt, 'General Serre weight conjectures', Preprint, 2015, arXiv:1509.02527.

[14] T. Gee and M. Kisin, 'The Breuil-Mézard conjecture for potentially Barsotti-Tate representations', Forum Math. Pi 2 (2014), e1 56; MR 3292675.

[15] T. Gee, T. Liu and D. Savitt, 'The Buzzard-Diamond-Jarvis conjecture for unitary groups', J. Amer. Math. Soc. 27(2) (2014), 389-435; MR 3164985.

[16] J. W. Jones and D. P. Roberts, 'A database of number fields', LMS J. Comput. Math. 17(1) (2014), 595-618; MR 3356048.

[17] C. Khare and J.-P. Wintenberger, 'Serre's modularity conjecture. I', Invent. Math. 178(3) (2009), 485-504; MR 2551763.

[18] C. Khare and J.-P. Wintenberger, 'Serre's modularity conjecture. II', Invent. Math. 178(3) (2009), 505-586; MR 2551764.

[19] L. Mavrides, 'On wild ramification in reductions of two-dimensional crystalline Galois representations'. PhD Thesis, King's College London, 2016.

[20] J. Newton, 'Serre weights and Shimura curves', Proc. Lond. Math. Soc. (3) 108(6) (2014), 1471-1500; MR 3218316.

[21] A. M. Robert, A Course in p-Adic Analysis, Graduate Texts in Mathematics, 198 (Springer, New York, 2000), MR 1760253.

[22] M. M. Schein, 'Weights in Serre's conjecture for Hilbert modular forms: the ramified case', Israel J. Math. 166 (2008), 369-391; MR 2430440.

[23] J.-P. Serre, Local Fields, Graduate Texts in Mathematics, 67 (Springer, New York-Berlin, 1979), translated from the French by Marvin Jay Greenberg; MR 554237.

[24] J.-P. Serre, 'Sur les représentations modulaires de degré 2 de $\operatorname{Gal}(\overline{\mathbf{Q}} / \mathbf{Q})$ ', Duke Math. J. 54(1) (1987), 179-230; MR 885783.

[25] R. Taylor, 'On Galois representations associated to Hilbert modular forms', Invent. Math. 98(2) (1989), 265-280; MR 1016264. 